\title{
Two-dimensional hydrodynamic core-collapse supernova simulations with spectral neutrino transport
}

\section{Numerical method and results for a $15 M_{\odot}$ star $^{\star}$}

\author{
R. Buras ${ }^{1,2}$, M. Rampp ${ }^{1, \star \star}$, H.-Th. Janka ${ }^{1}$, and K. Kifonidis ${ }^{1}$ \\ 1 Max-Planck-Institut für Astrophysik, Karl-Schwarzschild-Str. 1, 85741 Garching, Germany \\ e-mail: thj@mpa-garching.mpg.de \\ 2 Max-Planck-Institut für Physik, Föhringer Ring 6, 80805 München, Germany
}

Received 6 July 2005 / Accepted 18 October 2005

\section{ABSTRACT}

Supernova models with a full spectral treatment of the neutrino transport are presented, employing the PROMETHEUS/VERTEX neutrinohydrodynamics code with a variable Eddington factor closure of the $O(v / c)$ moments equations of neutrino number, energy, and momentum. Our "ray-by-ray plus" approximation developed for two- (or three-) dimensional problems assumes that the local neutrino distribution function is azimuthally symmetric around the radial direction, which implies that the nonradial flux components disappear. Other terms containing the angular velocity components are retained in the moments equations and establish a coupling of the transport at different latitudes by lateral derivatives. Also lateral components of the neutrino pressure gradients are included in the hydrodynamics equations. This approximative approach for neutrino transport in multi-dimensional environments is motivated and critically assessed with respect to its capabilities, limitations, and inaccuracies in the context of supernova simulations. In this first paper of a series, one- (1D) and two-dimensional (2D) core-collapse calculations for a (nonrotating) $15 M_{\odot}$ star are discussed, uncertainties in the treatment of the equation of state - numerical and physical - are tested, Newtonian results are compared with simulations using a general relativistic potential, bremsstrahlung and interactions of neutrinos of different flavors are investigated, and the standard approximation in neutrino-nucleon interactions with zero energy transfer is replaced by rates that include corrections due to nucleon recoil, thermal motions, weak magnetism, and nucleon correlations. Models with the full implementation of the "ray-by-ray plus" spectral transport were found not to explode, neither in spherical symmetry nor in 2D when the computational grid is constrained to a lateral wedge $\left(< \pm 45^{\circ}\right)$ around the equator. The success of previous two-dimensional simulations with grey, flux-limited neutrino diffusion can therefore not be confirmed. An explosion is obtained in 2D for the considered $15 M_{\odot}$ progenitor, when the radial velocity terms in the neutrino momentum equation are omitted. This manipulation increases the neutrino energy density in the convective gain layer by about $20-30 \%$ and thus the integral neutrino energy deposition in this region by about a factor of two compared to the non-exploding 2D model with the full transport. The spectral treatment of the transport and detailed description of charged-current processes leads to protonrich neutrino-heated ejecta, removing the problem that previous explosion models with approximate neutrino treatment overproduced $N=50$ closed neutron shell nuclei by large factors.

Key words. supernovae: general - neutrinos - radiative transfer - hydrodynamics

\section{Introduction}

Convective processes and hydrodynamic instabilities play an important role in different regions of collapsing and exploding stars. (1) Inside the nascent neutron star, i.e. below and around the neutrinosphere, convection enhances the transport and release of neutrinos and thus can raise the neutrino luminosities with potentially helpful consequences for the delayed shock

\footnotetext{
* Appendices A-C are only available in electronic form at http://www.edpsciences.org

$\star \star$ Present address: Rechenzentrum der Max-Planck-Gesellschaft am Max-Planck-Institut für Plasmaphysik, Boltzmannstr. 2, 85748 Garching, Germany.
}

revival and the neutrino-driven explosion mechanism (e.g., Burrows 1987; Burrows \& Lattimer 1988; Miralles et al. 2000; Pons et al. 1999; Wilson \& Mayle 1988, 1993; Keil et al. 1996; Keil 1997); (2) in the neutrino-heating region between gain radius and stalled supernova shock, convective overturn was found to enhance the energy deposition by neutrinos and the energy transport to the shock. Therefore it allows for neutrinodriven explosions even if models in spherical symmetry fail (Herant et al. 1994; Burrows et al. 1995; Janka \& Müller 1996); (3) multi-dimensional processes in the outer layers of the exploding star destroy the onion-shell structure of the progenitor and are responsible for the anisotropies and macroscopic mixing of chemical elements which was observed in 
Supernova 1987A and other supernovae, and which possibly is the origin of many morphological features of supernova remnants (e.g., Kifonidis et al. 2003, and references therein).

The existence of convectively unstable stratifications in the supernova core had long been suspected on grounds of spherically symmetric models (e.g., Epstein 1979; Bethe et al. 1987; Burrows 1987; Bethe 1990) before confirmation became possible by multi-dimensional modeling with sufficient resolution.

The first such multi-dimensional simulations (Burrows \& Fryxell 1992; Janka \& Müller 1993; Müller 1993) still ignored neutrino effects, were able to follow the evolution for a very limited period of time only, and started from post-bounce models that were constructed artificially or were adopted from simulations based on different assumptions about the input physics. Therefore they were only suitable to demonstrate the possibility in principle, but otherwise showed the transient decay of an initially unstable postshock stratification and thus could not be considered as meaningful models of supernovae (cf. Bruenn \& Mezzacappa 1994). However, simulations including the effects of neutrino heating and cooling (Herant et al. 1992) and true core collapse environments (Janka \& Müller 1994, 1995, 1996), neutrino transport by grey, flux-limited diffusion (Herant et al. 1994; Burrows et al. 1995), multi-group 1D transport (Mezzacappa et al. 1998b), rotation (Fryer \& Heger 2000), and three-dimensional hydrodynamics (Fryer \& Warren 2002, 2004) confirmed robustly the development of convective overturn in the neutrino-heated layer just behind the stalled shock, although it is still a matter of debate whether the associated convective effects are sufficient for driving delayed explosions (Mezzacappa et al. 1998b).

The situation is more controversial for convective activity below the neutrinosphere. A detailed investigation of neutrino transport properties at the conditions inside nascent neutron stars (Bruenn et al. 2004) revealed no evidence for neutron finger instability as assumed by the Livermore group (Wilson \& Mayle 1988, 1993) to trigger neutrino-driven explosions by convectively enhanced neutrino fluxes. In the simulations of Janka \& Müller (1996) the use of an inner boundary condition with imposed neutrino luminosities certainly played a role, causing neutrino absorption and heating and thus convective energy transport in the neutron star layers around the inner boundary (neutrino diffusion was ignored!). This convection below the neutrinosphere extended outward to a region where cooling by neutrino losses was fast enough to take up the energy delivered by the convective transport. However, full-scale hydrodynamic models of nascent neutron stars in two dimensions including a simple description of grey, radial neutrino diffusion and its effects on lepton number and energy transport showed the rapid development of convection inside the protoneutron star (Burrows \& Fryxell 1993) and its persistence in a growing volume until at least one second in nonrotating as well as rotating cases (Keil et al. 1996; Keil 1997; Janka \& Keil 1998; Janka 2004, whose simulations covered the evolution until at most $1.2 \mathrm{~s}$ after bounce). A careful analysis (see Keil 1997) revealed that the exchange of energy and in particular of lepton number between moving fluid elements and their surroundings was important so that the convective mode could not be described by ideal Ledoux convection. Instead, it had the character of a doubly diffusive instability, which can occur at Ledoux stable conditions as discussed by Bruenn \& Dineva (1996); Bruenn et al. (1995) and more recently in much detail by Bruenn et al. (2004).

This importance of entropy and lepton exchange of buoyant fluid elements with their surroundings via neutrino transport was also pointed out by Mezzacappa et al. (1998a). The latter authors, moreover, argued on the basis of their numerical and analytic models and timescale analyses that fast equilibration by neutrino transport tends to damp or even suppress entropyand lepton-driven proto-neutron star convection around and somewhat below the neutrinosphere, making it inefficient in the transport of lepton number and energy. The authors, however, admit that their investigations lack multi-dimensional "realistic" multi-group neutrino transport, coupled self-consistently to multi-dimensional hydrodynamics, which have to be applied in time-dependent evolution simulations of supernova cores before final conclusions can be drawn about the development of convection in optically thick regions. Bruenn et al. (2004) in particular stress the importance of a careful inclusion of neutrino transport effects for a reliable description of doubly diffusive instabilities.

While the basic results of the models of Keil et al. (1996) might therefore still withstand critical assessment by future, better simulations, quantitative aspects (e.g., the speed at which the convective layer grows inward towards the stellar center and enhances the deleptonization and energy loss) were undoubtedly affected by the simplicity of the treatment of the neutrino physics. Keil et al. (1996) used the assumption that neutrinos are in equilibrium with the stellar fluid (neutrino energy and pressure were therefore added to the corresponding gas quantities), that neutrino transport can be approximated by first-order grey, (flux-limited) radial equilibrium diffusion (cf. Mihalas \& Mihalas 1984, Sect. 97), and that the diffusive fluxes of lepton number and energy can be combined for electron neutrinos and antineutrinos into a gradient form with a single, average diffusion coefficient (cf. Appendix A of Keil \& Janka 1995). The need for removing these deficiencies has motivated new efforts for improved modeling, which were recently reported by Swesty \& Myra (2005).

In addition to Ledoux convection and doubly diffusive instabilities another form of multi-dimensional hydrodynamic instability seems to play a role in the supernova core: while one-dimensional, time-dependent hydrodynamic simulations (without neutrino transport) found that the standing accretion shock is stable against radial perturbations, two- and threedimensional (adiabatic) simulations demonstrated that it is unstable to non-radial modes, which lead to rapid growth of turbulence behind the shock and thus shock expansion and deformation (Blondin et al. 2003; Mezzacappa \& Blondin 2003). Such a standing accretion shock instability, SASI, was actually predicted for accreting black holes by analytic considerations by Foglizzo (2001, 2002), who described its origin from an amplifying feedback cycle of entropy and vorticity perturbations, which are advected inward and trigger acoustic waves that propagate outward and distort the shock, thus closing the cycle. The existence of this phenomenon at non-adiabatic conditions in the postshock flow was confirmed by supernova 
simulations including a simplified treatment of neutrino transport (Scheck et al. 2004; Scheck 2005). Its action can clearly be seen in particular if neutrino heating is too weak to drive strong postshock convection. In agreement with the adiabatic models of Foglizzo $(2001,2002)$ and Blondin et al. (2003), Scheck et al. (2004) find highest growth rates for low $(l=1,2)$ modes, which produce large, global asymmetries of the supernova explosion and might explain the observed pulsar kick velocities and grossly asymmetric distribution of elements seen in supernova remnants like Cassiopeia A (see Janka et al. 2005c).

Advancing core collapse supernova modeling to the next level of complexity and reliability beyond the stage of merely demonstrating possibilities in principle, requires improvements in the handling of neutrino transport coupled to multidimensional hydrodynamic simulations. Such improvements are necessary to clarify which role hydrodynamic instabilities play in the supernova core and how these are linked to the mechanism of the explosion. Crucial questions in this context are: does convection take place below the neutrinosphere and if so, which kind of instability is it? Does proto-neutron star convection raise the neutrinospheric luminosities and thus the neutrino heating behind the shock on timescales relevant for the explosion? Does neutrino-driven convection in the heating layer become sufficiently strong to trigger the explosion? Can the positive results of previous simulations with simplified neutrino transport by grey, flux-limited diffusion (Herant et al. 1994; Fryer 1999; Fryer \& Heger 2000; Fryer \& Warren 2002, 2004) be confirmed? Is the accretion shock instability found by Blondin et al. (2003) important and how does it interact with convective instabilities and rotation?

With the aim to address these questions in a next generation of core collapse studies, we have developed a new method for treating time- and frequency-dependent neutrino transport in multi-dimensional (in this work two-dimensional) environments. Our transport code is a generalization of the 1D version VERTEX of Rampp \& Janka (2002), adopting the approximations to general relativity described in the latter paper and tested for spherically symmetric problems against fully relativistic calculations in Liebendörfer et al. (2005) and Marek et al. (2006). The code employs a detailed spectral description of neutrino-matter interactions with fully implicit energy-bin coupling and with a set of processes which was updated from Rampp \& Janka (2002) by including interactions between neutrinos of different flavors and improving neutrino-nucleon interactions such that the effects of nucleon thermal motions, recoil, weak magnetism, and correlations in charged and neutral current processes are taken into account. The high dimensionality of the spatial and momentum dependence of the phase space distribution function is reduced by assuming the neutrino intensity to be axially symmetric around the radial direction. Consistent with this, we ignore lateral flux components and effects of neutrino viscosity and come up with a set of moments equations for neutrino number density, energy density and radial flux, which include all velocity-dependent terms of $O(v / c)$ for radial and lateral velocity components. These equations are solved in each angular bin of the spatial grid, taking into account the lateral (and in 3D also azimuthal) coupling by corresponding derivative terms in an operator splitting step ("ray-by-ray plus" approach). Closure of the set of moments equations is achieved by using variable Eddington factors, which are computed from a model Boltzmann equation as described in Rampp \& Janka (2002). The efficiency of the code performance is increased by determining these normalized moments of the neutrino intensity, which usually exhibit relatively weak variability with lateral direction, only once for all latitudes on grounds of an angularly averaged stellar background. Because of the fact that we refer to the solution of the model Boltzmann equation for closing the set of moments equations, we named our code MUDBATH (Multi-Dimensional Boltzmann Transport and Hydrodynamics).

Our approach to deal with neutrino transport in two- (and in an analogous way in three-) dimensional environments is intended to be a valid approximation for situations where only local macroscopic inhomogeneities are present, e.g. due to convection. It is, however, likely to become poor when a large global deformation (e.g. by rapid rotation) develops and the neutrino flow is therefore not well described by an at least on average dominant radial component. Our approach is complementary concerning the employed approximations to that of Livne et al. (2004), who introduced a scheme for solving the frequency-dependent Boltzmann transport equation in axially symmetric problems, but sacrificed energy-bin coupling and the majority of the terms with dependence on the motion of the stellar plasma. It also differs from the two-dimensional multi-group flux-limited diffusion approximation employed by Walder et al. (2005), who abandon energy-bin coupling and velocity-dependent terms, too.

In this Paper I and a subsequent one (Buras et al. 2005, Paper II) we present the first two-dimensional hydrodynamic simulations with a full spectral implementation of neutrino transport, applied to the core collapse and post-bounce evolution of progenitor stars with different masses (in the range from $11.2 M_{\odot}$ to $25 M_{\odot}$ ), compared to corresponding simulations in spherical symmetry. In Paper I we shall describe in detail our method for neutrino transport in two spatial dimensions, discuss numerical aspects and tests of the code and input physics, and try to assess the limitations of our method. We will concentrate on the presentation of numerical results for the evolution of a $15 M_{\odot}$ progenitor star (Model s15s7b2 of Woosley \& Weaver 1995) with varied input physics. For the 2D (axially symmetric) simulations we confine ourselves to using a roughly 90 degree angular wedge around the equatorial plane and will focus our discussion on neutrino-driven convection in the hot bubble region and its effect on the supernova dynamics. In Paper II we shall present and compare 1D and 2D simulations for different progenitors, using different angular wedges and resolution (including a model with a full $180^{\circ}$ polar grid) and considering also core rotation. Paper II will contain a detailed analysis of the importance of convection below the neutrinosphere relative to convection in the neutrino-heating layer, and of the role of low-mode instabilities, pre-collapse perturbations in the stellar core, and rotation in the investigated models. Since our spectral treatment of neutrino transport in the 2D simulations, which cover evolution periods of up to $300 \mathrm{~ms}$ after core bounce, is very CPU-time consuming - one model requires $(2-5) \times 10^{17}$ floating point 
operations, depending on the angular resolution and wedge size -, computer resources available to us prevented us from performing simulations with a systematic variation of important but not finally determined degrees of freedom, e.g., of the magnitude and distribution of angular momentum in the progenitor core. We therefore are forced to constrain our discussion on some selective cases.

The present paper is organized as follows. Section 2 (supplemented by Appendices A-C) contains a description of the employed equations, numerical algorithm, and tests of the numerical scheme and input physics, in particular of the equation of state. Section 3 presents results for 1D (Sect. 3.1) and 2D (Sect. 3.2) core collapse and post-bounce simulations of a $15 M_{\odot}$ progenitor with Newtonian gravity and with our approximative treatment of the effects of relativistic gravity, varied neutrino-matter interactions, and neutrino transport with and without taking into account the velocitydependent terms in the neutrino momentum equation. Thus retreating from our most complete implementation of neutrino transport by neglecting these velocity-dependent terms, we get cases with interesting behaviour, which once more demonstrate the sensitivity of the supernova evolution to supposedly secondary changes of neutrino transport: in the 1D simulation (Sect. 3.1.4) we observe a large-amplitude oscillation of the neutrino-decoupling layer between neutrinosphere and accretion shock, which grows in amplitude until it leads to an explosion; this reminds us of the $\kappa$ mechanism of vibrational instability of stars. In the corresponding 2D simulation (Sect. 3.2.2) strong post-shock convection develops and triggers a neutrinopowered supernova explosion, whose properties will be compared to observational constraints. In contrast, both the 1D and 2D models with the full transport implementation (Sects. 3.1.1 and 3.2.1, respectively) do not develop explosions. In Sect. 4 we give a summary of our main results and draw conclusions.

\section{Governing equations and numerical implementation}

In order to be able to exploit symmetries of the problem we work in a system of spherical coordinates with radius $r$, latitudinal angle $\vartheta$, and longitudinal angle $\varphi$. For all simulations performed so far we have assumed that azimuthal symmetry holds with respect to the polar axis, but a generalization of the presented method to three-dimensional situations is straightforward.

Like the one-dimensional version documented by Rampp \& Janka (2002) the algorithm relies on an operator splitting approach which means that the coupled system of evolution equations is processed in two independent steps, a hydrodynamic step and a neutrino-transport/interaction step. In each timestep these two steps are solved subsequently.

\subsection{Hydrodynamics}

For an ideal (i.e. nonviscous) fluid characterized by the mass density $\rho$, the radial, lateral, and azimuthal components of the velocity vector $\left(v_{r}, v_{\vartheta}, v_{\varphi}\right)$, specific energy $\varepsilon=e+\frac{1}{2}\left(v_{r}^{2}+v_{\vartheta}^{2}+v_{\varphi}^{2}\right)$ with $e$ being the specific internal energy, and gas pressure $p$, the
Eulerian, nonrelativistic equations of hydrodynamics in spherical coordinates and azimuthal symmetry read:

$$
\begin{aligned}
\frac{\partial}{\partial t} \rho+\frac{1}{r^{2}} & \frac{\partial}{\partial r}\left(r^{2} \rho v_{r}\right)+\frac{1}{r \sin \vartheta} \frac{\partial}{\partial \vartheta}\left(\rho \sin \vartheta v_{\vartheta}\right)=0 \\
\frac{\partial}{\partial t}\left(\rho v_{r}\right) & +\frac{1}{r^{2}} \frac{\partial}{\partial r}\left(r^{2} \rho v_{r} v_{r}\right)+\frac{1}{r \sin \vartheta} \frac{\partial}{\partial \vartheta}\left(\rho \sin \vartheta v_{\vartheta} v_{r}\right) \\
& -\rho \frac{v_{\vartheta}^{2}+v_{\varphi}^{2}}{r}+\frac{\partial p}{\partial r}=-\rho \frac{\partial \Phi}{\partial r}+Q_{\mathrm{M}_{r}} \\
\frac{\partial}{\partial t}\left(\rho v_{\vartheta}\right) & +\frac{1}{r^{2}} \frac{\partial}{\partial r}\left(r^{2} \rho v_{r} v_{\vartheta}\right)+\frac{1}{r \sin \vartheta} \frac{\partial}{\partial \vartheta}\left(\rho \sin \vartheta v_{\vartheta} v_{\vartheta}\right) \\
& +\rho \frac{v_{r} v_{\vartheta}-v_{\varphi}^{2} \cot \vartheta}{r}+\frac{1}{r} \frac{\partial p}{\partial \vartheta}=-\frac{\rho}{r} \frac{\partial \Phi}{\partial \vartheta}+Q_{\mathrm{M} \vartheta} \\
\frac{\partial}{\partial t}\left(\rho v_{\varphi}\right) & +\frac{1}{r^{2}} \frac{\partial}{\partial r}\left(r^{2} \rho v_{r} v_{\varphi}\right)+\frac{1}{r \sin \vartheta} \frac{\partial}{\partial \vartheta}\left(\rho \sin \vartheta v_{\vartheta} v_{\varphi}\right) \\
& +\rho \frac{v_{r} v_{\varphi}+v_{\vartheta} v_{\varphi} \cot \vartheta}{r}=0 \\
\frac{\partial}{\partial t}(\rho \varepsilon)+ & \frac{1}{r^{2}} \frac{\partial}{\partial r}\left(r^{2}(\rho \varepsilon+p) v_{r}\right) \\
& +\frac{1}{r \sin \vartheta} \frac{\partial}{\partial \vartheta}\left((\rho \varepsilon+p) \sin \vartheta v_{\vartheta}\right)= \\
& -\rho\left(v_{r} \frac{\partial \Phi}{\partial r}+\frac{v_{\vartheta}}{r} \frac{\partial \Phi}{\partial \vartheta}\right)+Q_{\mathrm{E}}+v_{r} Q_{\mathrm{M}_{r}}+v_{\vartheta} Q_{\mathrm{M} \vartheta}
\end{aligned}
$$

where $\Phi$ denotes the gravitational potential of the fluid, and $\boldsymbol{Q}_{\mathrm{M}}=\left(Q_{\mathrm{M}_{r}}, Q_{\mathrm{M}_{\vartheta}}\right)$ and $Q_{\mathrm{E}}$ are the neutrino source terms for momentum transfer and energy exchange, respectively. Eqs. (1)-(5) are closed by the equation of state $(\mathrm{EoS})$ which yields the pressure $p$ for given $\rho, e$, and composition. In the case of nuclear statistical equilibrium (NSE) a third independent variable, the electron fraction $Y_{\mathrm{e}}$, is sufficient to characterize the composition. For this variable the evolution is computed according to a conservation equation

$$
\frac{\partial}{\partial t}\left(\rho Y_{\mathrm{e}}\right)+\frac{1}{r^{2}} \frac{\partial}{\partial r}\left(r^{2} \rho Y_{\mathrm{e}} v_{r}\right)+\frac{1}{r \sin \vartheta} \frac{\partial}{\partial \vartheta}\left(\sin \vartheta \rho Y_{\mathrm{e}} v_{\vartheta}\right)=Q_{\mathrm{N}}
$$

where the source term $Q_{\mathrm{N}} /\left(\rho / m_{\mathrm{by}}\right)$ is the rate of change of the net electron fraction (i.e. the number fraction of electrons minus that of positrons) due to emission and absorption of electron-flavor neutrinos, and $m_{\mathrm{by}}$ is the baryon mass. In case the medium is not in NSE, an equation like Eq. (6) has to be solved also for the abundance of each nucleus $k, Y_{k} \equiv n_{k} / n_{\mathrm{by}}$, using

$\frac{\partial}{\partial t}\left(\rho Y_{k}\right)+\frac{1}{r^{2}} \frac{\partial}{\partial r}\left(r^{2} \rho Y_{k} v_{r}\right)+\frac{1}{r \sin \vartheta} \frac{\partial}{\partial \vartheta}\left(\sin \vartheta \rho Y_{k} v_{\vartheta}\right)=R_{k}$,

where $n_{k}$ and $n_{\text {by }}$ are the number density of nucleus $k$ and the baryon number density, respectively, and $R_{k} \equiv \rho \delta Y_{k} / \delta t$, where $\delta Y_{k} / \delta t$ is a source term that describes the rate of composition changes by nuclear reactions for species $k$.

For the numerical integration of Eqs. (1)-(7) we employ the Newtonian finite-volume code PROMETHEUS (Fryxell et al. 1989, 2000), which was supplemented by additional problem specific features (Keil 1997) and the improvements described in Kifonidis et al. (2003). PROMETHEUS is a direct Eulerian implementation of the Piecewise Parabolic Method (PPM) of Colella \& Woodward (1984). As a time-explicit, third-order in space, second order in time Godunov scheme 
with a Riemann solver it is particularly well suited for following discontinuities in the fluid flow like shocks, contact discontinuities, or boundaries between layers of different chemical composition. A notable advantage in the present context is its capability of solving multi-dimensional problems with high computational efficiency and numerical accuracy. Our code makes use of the "Consistent Multifluid Advection" (CMA) method (Plewa \& Müller 1999) for ensuring an accurate advection of different chemical components of the fluid, and switches from the original PPM method to the HLLE solver of Einfeldt (1988) in the vicinity of strong shocks to avoid spurious oscillations (the so-called "odd-even decoupling", or "carbunkel", phenomenon) when such shocks are aligned with one of the coordinate directions in multidimensional simulations (Quirk 1994; Liou 2000; Kifonidis et al. 2003; Sutherland et al. 2003).

Although our hydrodynamic scheme is Newtonian, we have included effects of general relativistic (GR) gravity approximately in the following way: the gravitational potential used in our simulations can be symbolically written as $\Phi(r, \vartheta)=$ $\Phi_{2 \mathrm{D}}^{\mathrm{Newt}}(r, \vartheta)+\left(\Phi_{1 \mathrm{D}}^{\mathrm{GR}}(r)-\Phi_{1 \mathrm{D}}^{\mathrm{Newt}}(r)\right)$. We compute the Newtonian gravitational potential $\Phi_{2 \mathrm{D}}^{\text {Newt }}$ for the two-dimensional axisymmetric mass distribution by expanding the integral solution of the Poisson equation into a Legendre series, truncated at $l=10$ (cf. Müller \& Steinmetz 1995). General relativistic effects are approximately taken into account by the spherically symmetric correction term $\Phi_{1 \mathrm{D}}^{\mathrm{GR}}-\Phi_{1 \mathrm{D}}^{\mathrm{Newt}}$, where $\Phi_{1 \mathrm{D}}^{\mathrm{GR}}$ denotes an effective general relativistic gravitational potential as employed for spherically symmetric simulations (see Rampp \& Janka 2002, Eq. (53)) and $\Phi_{1 \mathrm{D}}^{\text {Newt }}$ is its Newtonian counterpart. The general relativistic potential $\Phi_{1 \mathrm{DR}}^{\mathrm{GR}}$ is deduced from a comparison of the Newtonian and relativistic equations of motion in spherical symmetry and includes terms due to the pressure and energy of the stellar medium and neutrinos (see Rampp \& Janka 2002). Both, $\Phi_{1 \mathrm{DR}}^{\mathrm{GR}}(r)$ and $\Phi_{1 \mathrm{D}}^{\text {Newt }}(r)$ are computed using angular averages of the evolved variables. In twodimensional simulations which cover only a limited range of latitudes $0<\vartheta_{\min } \leq \vartheta \leq \vartheta_{\max }<\pi$ around the equatorial plane we set $\Phi(r, \vartheta)=\Phi_{1 \mathrm{D}}^{\mathrm{GR}}(r)$.

The source terms $Q_{\mathrm{M} r}, Q_{\mathrm{M} \vartheta}, Q_{\mathrm{E}}$, and $Q_{\mathrm{N}}$ on the right-hand sides of Eqs. (2), (3), (5), (6) are determined by the solution of the neutrino transport equations. The source terms $R_{k}$ depend on changes of the composition according to nuclear burning. Unless stated otherwise, the EoS we apply is the same as described in detail in (Rampp \& Janka 2002, Appendix B).

Note that PROMETHEUS only solves the left-hand sides of the hydrodynamic Eqs. (1)-(7) and that the EoS is not evaluated during this procedure. The computation of the terms on the right-hand sides, i.e the gravitational, neutrino, and burning effects, as well as the evaluation of the EoS and, if necessary, the determination of the NSE composition, are done in operator split steps.

\subsection{Neutrino transport}

Solving the full two-dimensional neutrino transport equation would be the most precise way of simulating supernovae in two dimensions. On the level of the radiation moments equations this would, compared to the 1D case, lead to several new degrees of freedom and one additional moments equation, which would require five more closure relations to be introduced. Therefore we limit ourselves to a less ambitious extension of the one-dimensional moments equations to two dimensions in which new degrees of freedom (including, in case of the considered azimuthal symmetry, the lateral flux and off-diagonal pressure tensor terms which account for neutrino viscosity) are set to zero, but the additional moments equation (in our case for the lateral flux) is taken into account (in the sense that we retain the terms corresponding to the lateral neutrino pressure gradients at optically thick conditions). In the next section, we will justify why we believe this approximation is sufficient for simulating two-dimensional neutrino transport in the considered models of core-collapse supernovae, but also explain why further simplifications are not allowed.

The great advantage of our "ray-by-ray" 2D neutrino transport is that the neutrino moments equations at different latitudes (except for some terms which can be accounted for explicitly in an operator split) decouple from each other. Therefore, for each "radial ray", i.e. for all zones of same polar angle, the moments equations can be solved independently. Except for some additional terms this problem is identical to solving $N_{\vartheta}$ times the moments equations for a spherically symmetric star with $N_{\vartheta}$ being the number of grid zones in polar direction.

We further make the following standard assumptions: first, we ignore neutrino oscillations in the SN core, where neutrino effects are treated by solving the transport problem. This is justified if one ignores the results of LSND; then the parameters for atmospheric and solar neutrino oscillations predict the resonant MSW effect to be at densities far below $10^{5} \mathrm{~g} \mathrm{~cm}^{-3}$ so that this effect has no influence on the region of interest (for the possible importance of neutrino flavor conversions at high densities, see Fuller \& Qian 2005). Furthermore, nonresonant oscillations are strongly suppressed in the proto neutron star (PNS) due to first and second order refractive effects, see Hannestad et al. (2000). Second, the medium, even in the PNS, initially does not contain any muons and temperatures and densities are always too low to produce tauons, which implies small or vanishing chemical potentials for the $\mu$ and $\tau$ type neutrinos. Further, the opacities are nearly equal for $v_{\mu}, \bar{v}_{\mu}, v_{\tau}$, and $\bar{v}_{\tau}$. Therefore, we treat these four neutrino types identically and set $\mu_{\nu_{\mu}} \equiv \mu_{\nu_{\tau}} \equiv 0$. We will notate them collectively as " $v_{\mathrm{x}}$ ".

\subsubsection{Moments equations}

In a two-dimensional transport scheme assuming azimuthal symmetry, the specific intensity $\mathcal{I}(t, r, \vartheta, \varphi, \epsilon, \boldsymbol{n})$ does not depend on the azimuth $\varphi$. We describe the direction of propagation $\boldsymbol{n}$ by the angle cosine $\mu \equiv \boldsymbol{n} \cdot \boldsymbol{r} /|\boldsymbol{r}|$, measured with respect to the radius vector $\boldsymbol{r}$, and the angle $\omega$. Then azimuthal symmetry implies $\mathcal{I}(\ldots, \mu, \omega)=\mathcal{I}(\ldots, \mu,-\omega)$, see also Appendix B. Making the additional assumption that $I$ is independent of $\omega$, 
each of the angular moments of the specific intensity can be expressed by one scalar, namely

$$
\{J, H, K, L, \ldots\}(t, r, \vartheta, \epsilon) \equiv \frac{1}{2} \int_{-1}^{+1} \mathrm{~d} \mu \mu^{\{0,1,2,3, \ldots\}} \mathcal{I}(t, r, \vartheta, \epsilon, \mu)
$$

where we have used Eqs. (B.9)-(B.12) - which follow from our assumptions - to reduce the number of independent variables in the angular moments of the neutrino intensity as defined in Eqs. (B.3). As usual, $\epsilon$ denotes the energy of the neutrinos. As a consequence of the afore mentioned assumptions the set of moments equations for describing the evolution of neutrino energy and flux in the comoving frame, given by Eqs. (B.4)-(B.7) in the Newtonian, $O(v / c)$ approximation, simplifies to Eqs. (B.13) and (B.14) for the remaining independent variables $J$ and $H \equiv H_{r}$. In our approximation Eqs. (B.6) and (B.7) can be ignored because the variables the evolution of which they describe, i.e. $H_{\vartheta}$ and $H_{\varphi}$, are strictly set to zero. With $\mathcal{T}=J / \epsilon, \mathcal{H}=H / \epsilon, \mathcal{K}=K / \epsilon$, and $\mathcal{L}=L / \epsilon$, the moments equations describing the evolution of neutrino number are given by Eqs. (B.15) and (B.16).

The velocity-dependent terms, in the order of their appearance and grouping in Eqs. (B.13)-(B.16), correspond to the physical effects of radiation advection with the moving stellar fluid (expressed by terms containing $\beta_{r} \partial / \partial r$ and $\left(\beta_{\vartheta} / r\right) \partial / \partial \vartheta$ for the velocity components $v_{r}$ and $v_{\vartheta}$, respectively), radiation compression, Doppler shifting (within the energy derivatives), and finally the fluid acceleration. Note that in the current version of our computer code the $\beta_{r} \partial / \partial t$-derivatives of the moments, which appear at the end of the respective first lines of Eqs. (B.13)-(B.16), cf. Rampp \& Janka (2002), are ignored.

The system of moments Eqs. (B.13)-(B.16) is very similar to the Newtonian, $O(v / c)$ moments equations in spherical symmetry (see Rampp \& Janka 2002, Eqs. (7), (8), (30), (31)). We have set the additional terms arising from our approximative generalization to two dimensions in boldface. Adding general relativistic (GR) effects in the spirit of Rampp \& Janka (2002) to our approximative 2D transport proves not to alter the boldface terms, so that the corresponding equations are Eqs. (54)-(57) in the latter paper, which handle the GR effects for 1D transport, plus those terms in Eqs. (B.13)-(B.16) that are typeset in boldface. The equations are closed by substituting $K=f_{K} \cdot J$ and $L=f_{L} \cdot J$, where $f_{K}$ and $f_{L}$ are the variable Eddington factors.

In order to discretize Eqs. (B.13)-(B.16), the computational domain $\left[0, r_{\max }\right] \times\left[\vartheta_{\min }, \vartheta_{\max }\right]$ is covered by $N_{r}$ radial and $N_{\vartheta}$ angular zones, where $\vartheta=0, \pi$ correspond to the polar axis and $\vartheta=\pi / 2$ to the equatorial plane of the spherical grid. All neutrino variables are defined on the angular centres of the zones with the coordinate $\vartheta_{k+\frac{1}{2}} \equiv \frac{1}{2}\left(\vartheta_{k}+\vartheta_{k+1}\right)$ being defined as the arithmetic mean of the corresponding interface values. The equations are solved in two operator-split steps corresponding to a lateral and a radial sweep.

In a first step, we treat the boldface terms in the respectively first lines of Eqs. (B.13)-(B.16), which describe the lateral advection of the neutrinos with the stellar fluid, and thus couple the angular moments of the neutrino distribution of neighbouring angular zones. For this purpose we solve the equation

$$
\frac{1}{c} \frac{\partial \Xi}{\partial t}+\frac{1}{r \sin \vartheta} \frac{\partial\left(\sin \vartheta \beta_{\vartheta} \Xi\right)}{\partial \vartheta}=0
$$

where $\Xi$ represents one of the moments $J, H, \mathcal{J}$, or $\mathcal{H}$. After integration over the volume of a zone $\left(i+\frac{1}{2}, k+\frac{1}{2}\right)$, where $i$ and $k$ are the indices of the radial and lateral zones, the finitedifferenced version of Eq. (9) reads:

$$
\begin{aligned}
\frac{\Xi_{i+\frac{1}{2}, k+\frac{1}{2}}^{n+1}-\Xi_{i+\frac{1}{2}, k+\frac{1}{2}}^{n}}{c t^{n+1}-c t^{n}} & \\
+\frac{1}{\Delta V_{i+\frac{1}{2}, k+\frac{1}{2}}} & \left(\Delta A_{i+\frac{1}{2}, k+1} \beta_{\vartheta_{i+\frac{1}{2}, k+1}} \Xi_{i+\frac{1}{2}, k_{i+1 / 2}(k+1)}^{n}\right. \\
& \left.-\Delta A_{i+\frac{1}{2}, k} \beta_{\vartheta_{i+\frac{1}{2}, k}} \Xi_{i+\frac{1}{2}, k_{i+1 / 2}(k)}^{n}\right)=0,
\end{aligned}
$$

with the volume element $\Delta V_{i+\frac{1}{2}, k+\frac{1}{2}}=\frac{2 \pi}{3}\left(r_{i+1}^{3}-r_{i}^{3}\right)\left(\cos \vartheta_{k}-\right.$ $\left.\cos \vartheta_{k+1}\right)$ and the surface element $\Delta A_{i+\frac{1}{2}, k}=\pi\left(r_{i+1}^{2}-r_{i}^{2}\right) \sin \vartheta_{k}$. Note that additional indices of $\Xi$ which label energy bins and the different types of neutrinos are suppressed for clarity. To guarantee monotonicity, upwind values of the moments $\Xi_{i+\frac{1}{2}, \kappa_{i+1 / 2}(k)}^{n}$, with

$\kappa_{i+1 / 2}(k) \equiv\left\{\begin{array}{l}k-\frac{1}{2} \text { for } \beta_{\vartheta}{ }_{i+\frac{1}{2}, k}>0, \\ k+\frac{1}{2} \text { else, }\end{array}\right.$

are used for computing the lateral fluxes across the interfaces of the angular zones. The time-step limit enforced by the CourantFriedrichs-Lewy (CFL) condition is

$\Delta t_{\mathrm{CFL}}=\min _{i, k} \frac{\Delta x_{\vartheta ; i+\frac{1}{2}, k+\frac{1}{2}}}{\left|v_{\vartheta ; i+\frac{1}{2}, k+\frac{1}{2}}\right|}$

where $\Delta x_{\vartheta}$ is the zone width in lateral direction. It turns out that this condition is not restrictive in our simulations. In practice, the numerical time-step is always limited by other constraints.

Now, in the second step, the radial sweep for solving Eqs. (B.13)-(B.16) is performed. Considering a radial ray with given $\vartheta_{k+\frac{1}{2}}$, the radial discretization of the equations (and of their general relativistic counterparts) proceeds exactly as detailed in Rampp \& Janka (2002). The terms in boldface not yet taken into account in the lateral sweep do not couple the the neutrino distribution function of neighbouring angular zones and thus can be included into the discretization scheme of the radial sweep in a straightforward way.

\subsubsection{Eddington factors}

In analogy to the treatment of the moments equations in the previous subsection the calculation of the variable Eddington factors could in principle also be done on (almost) decoupled radial rays. However, such a procedure would require a sizeable amount of computer time. On the other hand, the Eddington factors are normalized moments of the neutrino phase space distribution function and thus, in the absence of persistent global deformation of the star, should not show significant variation with the angular coordinate (cf. Rampp 2000; 
Rampp \& Janka 2002). Therefore we have decided to determine the variable Eddington factors only once for an "angularly averaged" radial ray instead of computing them for each radial ray separately. The corresponding reduction of the computational load can be up to a factor of 10 (Rampp \& Janka 2002).

In analogy to the spherically symmetric case the code solves a time-step of the one-dimensional neutrino transport on a spherically symmetric image of the stellar background. The latter is defined as the angular averages of the structure variables $\xi \in\left\{\rho, T, Y_{\mathrm{e}}, \beta_{r}, \ldots\right\}$ according to $\xi(t, r) \equiv$ $\frac{1}{b-a} \int_{a}^{b} \mathrm{~d} \cos \vartheta \xi(t, r, \vartheta) w(t, r, \vartheta)$, where $a=\cos \left(\vartheta_{\max }\right)$ and $b=$ $\cos \left(\vartheta_{\min }\right)$ and $w$ is a weighting function which can be either $\rho$ or 1 depending on which choice is more appropriate. The computation of the 1D transport step proceeds exactly as described for the spherically symmetric version of the code (see Rampp \& Janka 2002), i.e. the coupled set of the 1D moments equations and a 1D model Boltzmann equation are iterated to convergence to obtain solutions for the variable Eddington factors $f_{\mathrm{K}}$ and $f_{\mathrm{L}}$. These variable Eddington factors are used for all latitudes $\vartheta$ of the multidimensional transport grid when solving the two-dimensional moments equations. We will discuss and try to estimate the possible errors associated with our approximate 2D transport treatment in Sect. 2.3.

\subsubsection{Coupling to the hydrodynamics}

The system of neutrino transport Eqs. (B.13)-(B.16) is coupled with the equations of hydrodynamics (1-6) by virtue of the source terms

$$
\begin{aligned}
Q_{\mathrm{N}} & =\sum_{v}-4 \pi m_{\text {by }} \operatorname{sgn}(v) \int_{0}^{\infty} \mathrm{d} \epsilon \mathfrak{C}_{v}^{(0)}(\epsilon), \\
Q_{\mathrm{E}} & =\sum_{v}-4 \pi \int_{0}^{\infty} \mathrm{d} \epsilon C_{v}^{(0)}(\epsilon), \\
Q_{\mathrm{M} r} & =\sum_{v}-\frac{4 \pi}{c} \int_{0}^{\infty} \mathrm{d} \epsilon C_{r, v}^{(1)}(\epsilon), \\
Q_{\mathrm{M}_{\vartheta}} & =\sum_{v}-\frac{4 \pi}{c} \int_{0}^{\infty} \mathrm{d} \epsilon C_{\vartheta, v}^{(1)}(\epsilon),
\end{aligned}
$$

where $m_{\text {by }}$ denotes the baryon mass, $\mathfrak{c}_{v}^{(0)}(\epsilon) \equiv \epsilon^{-1} C_{v}^{(0)}(\epsilon)$, and $C_{\nu}^{(0)}(t, r, \vartheta, \epsilon) \equiv(4 \pi)^{-1} \int \mathrm{d} \Omega C_{v}(t, r, \vartheta, \epsilon, \boldsymbol{n})$ and $\boldsymbol{C}_{v}^{(1)}(t, r, \vartheta, \epsilon)=$ $\left(C_{r, v}^{(1)}, C_{\vartheta, v}^{(1)}\right)=(4 \pi)^{-1} \int \mathrm{d} \Omega \boldsymbol{n} C_{v}(t, r, \vartheta, \epsilon, \boldsymbol{n})$ are angular moments of the collision integral of the Boltzmann equation, $C_{v}$. Note that in Eqs. (13)-(16) the moments of the collision integral are summed over all neutrino types $v \in$ $\left\{v_{\mathrm{e}}, \bar{v}_{\mathrm{e}}, v_{\mu}, \bar{v}_{\mu}, v_{\tau}, \bar{v}_{\tau}\right\}$, and $\operatorname{sgn}(v)=+1$ for neutrinos and -1 for antineutrinos. Remember that we treat $v_{\mu}, \bar{v}_{\mu}, v_{\tau}$, and $\bar{v}_{\tau}$ identically because their matter interactions are nearly equal. They do not transport electron lepton number and therefore do not contribute to $Q_{\mathrm{N}}$. In the following we suppress the index $v$.

Our simplification of the neutrino transport equations enforces a radial flux vector, i.e. the angular flux component $H_{\vartheta} \equiv 0$. However, as we shall demonstrate in Sect. 2.3.2, the corresponding lateral component of the momentum transfer from neutrinos to the stellar medium, described by the source term $Q_{\mathrm{M} \vartheta}$, can not be neglected in the Euler equation of the stellar fluid, Eq. (3), when the neutrinos are tightly coupled to the medium. This implies that we should solve the moments equation for the lateral transport of neutrino momentum (B.6) which, using the assumptions Eqs. (B.9)-(B.12), simplifies to

$$
\begin{aligned}
C_{\vartheta}^{(1)}(\epsilon)= & \left(\frac{\partial \beta_{\vartheta}}{\partial r}-\frac{\beta_{\vartheta}}{r}\right) H+\frac{1}{2 r} \frac{\partial(J-K)}{\partial \vartheta}+\frac{1}{2 c} \frac{\partial \beta_{\vartheta}}{\partial t}(3 J-K) \\
& +\frac{\beta_{\vartheta}}{2 c} \frac{\partial(J-K)}{\partial t}-\frac{\partial}{\partial \epsilon}\left\{\epsilon \left[\frac{1}{2 c} \frac{\partial \beta_{\vartheta}}{\partial t}(J-K)\right.\right. \\
& \left.\left.+\frac{1}{2}\left(\frac{\partial \beta_{\vartheta}}{\partial r}+\frac{1}{r} \frac{\partial \beta_{r}}{\partial \vartheta}-\frac{\beta_{\vartheta}}{r}\right)(H-L)\right]\right\}
\end{aligned}
$$

On the other hand, at the conditions present in the optically thick PNS an isotropic neutrino distribution (i.e. $J \equiv 3 K$ ) and diffusion are good approximations. Concerning the velocity dependent first term in Eq. (17), which is of order $\frac{v}{c} \frac{\lambda}{r}$ where $\lambda$ is the neutrino mean free path, see the discussion about first order and 2nd order diffusion in Mihalas \& Mihalas (1984, Sect. 97, p. 458ff). Omitting this term means that we ignore all effects of neutrino viscosity. Thus the above equation simplifies considerably to (assuming also stationary conditions, i.e. $\partial / \partial t \equiv 0$ )

$\int_{0}^{\infty} \mathrm{d} \epsilon C_{\vartheta}^{(1)}(\epsilon)=\int_{0}^{\infty} \mathrm{d} \epsilon \frac{1}{2 r} \frac{\partial(J-K)}{\partial \vartheta}=\int_{0}^{\infty} \mathrm{d} \epsilon \frac{1}{3 r} \frac{\partial J}{\partial \vartheta}$,

where the terms with the energy derivative in Eq. (17) vanish when integrating over the neutrino energy. Given $J(r, \vartheta)$ as the solution of the moments Eqs. (B.13), (B.14), Eq. (18) together with the definition Eq. (16) allows us to compute an approximation for the momentum exchange rate, $Q_{\mathrm{M}_{\vartheta}}$, between neutrinos and the stellar fluid.

Finally, Eq. (B.7) would result in a momentum transfer from the neutrinos to the medium in azimuthal direction, $C_{\varphi}^{(1)}$, in the presence of rotation, $\beta_{\varphi} \neq 0$. Using our usual assumptions Eqs. (B.9)-(B.12) we obtain for Eq. (B.7)

$$
\begin{aligned}
C_{\varphi}^{(1)}(\epsilon)= & \left(\frac{\partial \beta_{\varphi}}{\partial r}-\frac{\beta_{\varphi}}{r}\right) H+\frac{1}{2 c} \frac{\partial \beta_{\varphi}}{\partial t}(3 J-K)+\frac{\beta_{\varphi}}{2 c} \frac{\partial(J-K)}{\partial t} \\
& -\frac{\partial}{\partial \epsilon}\left\{\epsilon \left[\frac{1}{2 c} \frac{\partial \beta_{\varphi}}{\partial t}(J-K)\right.\right. \\
& \left.\left.+\frac{1}{2}\left(\frac{\partial \beta_{\varphi}}{\partial r}-\frac{\beta_{\varphi}}{r}\right)(H-L)\right]\right\} .
\end{aligned}
$$

However, again assuming diffusion, stationary conditions, and ignoring neutrino viscosity, all terms of this expression vanish.

The numerical discretization of Eq. (18) reads

$$
C_{\vartheta}^{(1)}{ }_{i+\frac{1}{2}, k+\frac{1}{2}}=\frac{J_{i+\frac{1}{2}, k+\frac{3}{2}}-J_{i+\frac{1}{2}, k-\frac{1}{2}}}{3 r_{i+\frac{1}{2}}\left(\vartheta_{k+\frac{3}{2}}-\vartheta_{k-\frac{1}{2}}\right)}
$$

Since Eq. (18) is valid only in the limit of an optically thick medium we set $C_{\vartheta}^{(1)}{ }_{i+\frac{1}{2}, k+\frac{1}{2}}=0$ if the density $\rho_{i+\frac{1}{2}, k+\frac{1}{2}}$ in a zone drops below $10^{12} \mathrm{~g} \mathrm{~cm}^{-3}$. The chosen cut-off value is obviously specific to the core-collapse supernova problem where outside of this density the neutrino pressure gradients, in particular in the lateral direction, turn out to be negligibly small. 


\subsection{Discussion and tests of the numerical scheme}

In the last subsection we described our implementation of an approximative neutrino transport scheme for two-dimensional configurations using spherical coordinates and azimuthal symmetry.

Besides adopting the approximations of general relativistic effects from the spherically symmetric VERTEX code of Rampp \& Janka (2002) we made two major approximations of the transport equations. First, the dependence of the specific intensity on the direction of propagation $\boldsymbol{n}$ is replaced by a dependence on only one angle cosine $\mu$. Secondly and closely related to the first approximation, we use scalar variable Eddington factors. These are obtained from the solution of the one-dimensional transport equations on a spherically symmetric image of the stellar background. Note, however, that our treatment described here has been extended considerably by additional terms (cf. Sects. 2.2.1, 2.2.3) compared to the simpler ray-by-ray transport scheme suggested in Rampp \& Janka (2002, Sect. 3.8).

In the following we point out limitations of our approach and try to critically assess their influence on the results obtained with our method.

\subsubsection{Treatment of general relativity}

The radial neutrino transport contains gravitational redshift and time dilation, but ignores the distinction between coordinate radius and proper radius. This simplification is necessary for consistently coupling the transport code to our basically Newtonian hydrodynamics. Of course, one would ultimately have to work in a genuinely multi-dimensional GR framework which, among other complications (see e.g. Cardall \& Mezzacappa 2003), entails abandoning the use of the Lindquist metric.

Tests showed that in spherically symmetric simulations our approximations seem to work satisfactorily well (Liebendörfer et al. 2005; Marek et al. 2006), at least as long as the infall velocities do not reach more than $10-20 \%$ of the speed of light in decisive phases of the evolution. Unless very extreme conditions are considered (e.g. very rapid rotation) gravity in supernovae is dominated by radial gradients. We therefore expect that effects from a fully multi-dimensional GR treatment are small and disregarding them is acceptable in view of the other approximations made in the multi-dimensional treatment of the transport. Some tests of applicability of our 2D approximation of relativistic gravity can be found in Marek et al. (2006).

It should be noted that the effective relativistic gravitational potential which we use in multi-dimensional simulations (see Sect. 2.1) cannot guarantee strict conservation of momentum (for a detailed explanation see Marek et al. 2006). However, in practical applications the size of the violation turns out to be very small, see Fig. 1. In a $2 \mathrm{D}$ model with lateral periodic boundary conditions, Model s15Gio_32.b, the total momentum of the matter on the computational grid (which should remain zero) reaches moderate values (corresponding to velocities of at most $100 \mathrm{~km} \mathrm{~s}^{-1}$ for a mass of $1 M_{\odot}$ ). However, due to the periodic boundary conditions the center of mass does not

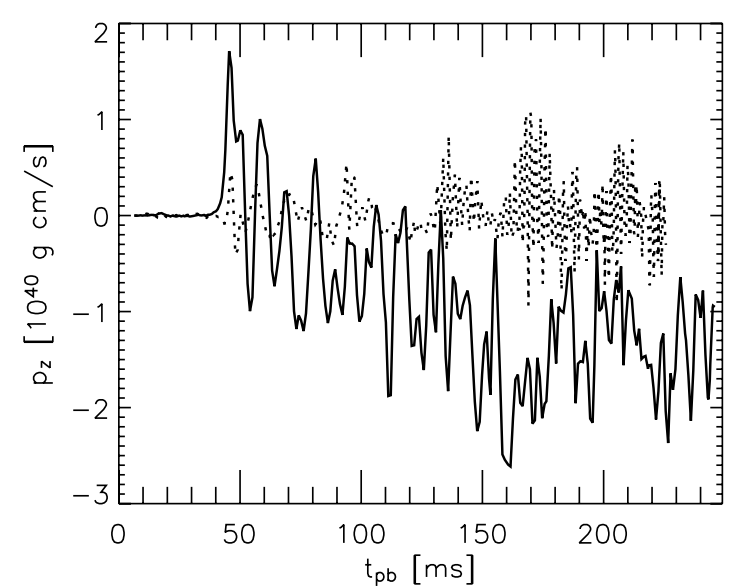

$\mathbf{a}$

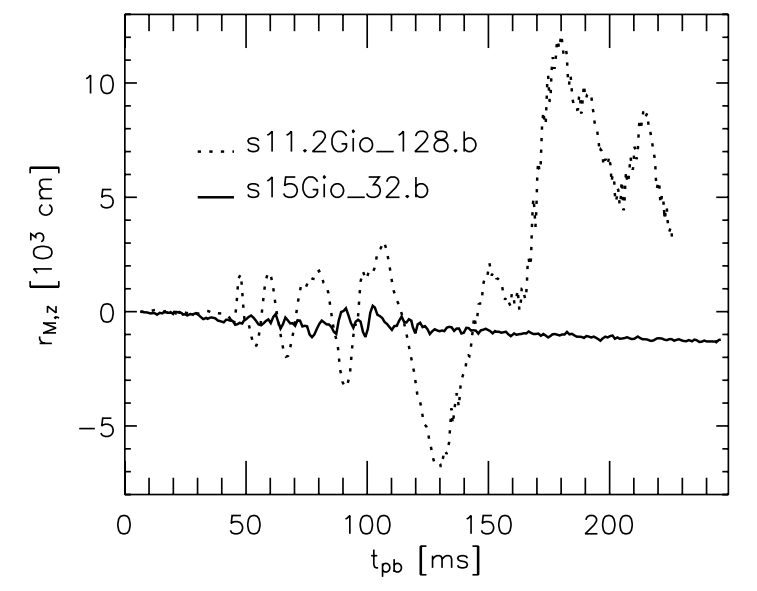

Fig. 1. a) Component of the linear momentum in polar axis direction of all matter on the computational grid. The solid line shows its time evolution for Model s15Gio_32.b, in which a lateral wedge around the equator and with periodic boundary conditions was used (this model is described in detail in Sect. 3.2.1). The dotted line belongs to Model s11.2Gio_128.b, which is a model with a full $180^{\circ}$ grid and thus with reflecting boundary conditions at the poles (in lateral direction); this model will be described in Buras et al. (2005). b) Distance between the center of the grid and the center of mass for the same two models.

move significantly away from the center of the grid because the matter which flows out of the grid through one lateral boundary reappears at the opposite grid boundary. In a model with lateral reflecting boundary conditions, Model s11.2Gio_128.b (in this model the lateral grid reaches from pole to pole; this model will be described in detail in Buras et al. 2005), no such shift of mass occurs, so that the center of mass moves according to the total momentum. However, as can be seen in Fig. 1, for this model the total momentum remains much smaller than in Model s15Gio_32.b (corresponding to velocities of at most $50 \mathrm{~km} \mathrm{~s}^{-1}$ for $1 M_{\odot}$ ), and the time average of the velocity is close to zero. Consequently, the distance between the center of the grid and the center of mass remains far below $1 \mathrm{~km}$. This behaviour is explained by the fact that the direction of the artificial "force" creating the violation of momentum will usually be from the center of mass towards the center of the grid. In summary, the nonconservation of total momentum due to the employed treatment of the effective relativistic 
gravitational potential remains a very small perturbation which has no influence on our simulations.

\subsubsection{Lateral coupling and neutrino pressure gradients}

Including the terms printed in boldface in Eqs. (B.13)-(B.16) and taking into account the acceleration of the stellar fluid in the lateral direction by neutrino pressure gradients, we extend our non-equilibrium transport scheme beyond a simple ray-byray description. This extension is consistent with our fundamental assumption of azimuthal symmetry of the intensity $\mathcal{I}$ (which implies $H_{\vartheta} \equiv 0$ ) and makes sure that our transport description produces the correct behaviour in important limiting cases.

First, in optically thick regions where neutrinos are tightly coupled to the stellar fluid, neutrinos must be allowed to be carried along with (laterally) moving fluid elements. This assures the conservation of the total lepton number $\left(Y_{\text {lep }}=Y_{\mathrm{e}}+Y_{v}\right)$ in these fluid elements in the absence of neutrino transport relative to the medium $\left(D Y_{\text {lep }} / D t=0\right.$ for the Lagrangian time derivative). Below we will demonstrate that if this conservation is violated by omitting the terms describing lateral advection, radiation compression, and Doppler effects in the neutrino moments equations, fluctuations in the lepton number are artificially induced, which grow and trigger macroscopic fluid motions, e.g., by buoyancy-driven rise of high- $Y_{\mathrm{e}}$ domains. Secondly, when neutrinos yield a significant contribution to the pressure (as is the case in the dense interior of the hot, nascent neutron star) the inclusion of lateral neutrino pressure gradients is again important to prevent artificial acceleration of the fluid by gradients of the gas pressure and to provide a restoring force which damps the motion of the fluid when it transports neutrinos from one place to another.

In contrast, the omission of angular flux components $\left(H_{\vartheta}=\right.$ 0 ) means the disregard of "active propagation" of neutrinos relative to the stellar fluid. Although it is not clear that this is a good assumption (for example, the effects of neutrino diffusion might be underestimated, see below), it is unlikely to lead to fundamental inconsistencies, because it is the correct physical limit for situations where the opacity is very high. In the same spirit also off-diagional terms of the neutrino pressure tensor $\left(P_{i j}\right.$ with $\left.i \neq j\right)$ can be dropped, implying that effects of neutrino viscosity are ignored.

We point out here that another inconsistency is imported into our treatment of 2D transport. The omission of angular flux components causes the problem that the correct limit at large radii and small optical depth may not be accurately reproduced because the evolution of the radiation moments $J$ and $H$ even at large radius depends on lateral gradient terms that include $\beta_{\vartheta}$. Fortunately, the specifics of the supernova problem help us justifying our approach: at large radii and low optical depth the lateral component $v_{\vartheta}$ of the fluid velocity is usually small (in particular relative to the radial component of the velocity, which determines relevant timescales) so that all terms scaling with $\beta_{\vartheta}=v_{\vartheta} / c$ become of minor importance. Secondly, multi-dimensional effects of neutrino transport usually lead to a neutrino distribution outside of the neutrinosphere, which is more isotropic than the stellar structure (Livne et al. 2004), an effect which our transport treatment tends to underestimate anyway. Thirdly, at large radii the non-cancelling $\beta_{\vartheta}$-terms are suppressed by a factor $1 / r$, and the small remaining lateral redistribution of the neutrino flux is irrelevant because the coupling between neutrinos and stellar medium (e.g. neutrino heating and cooling) vanishes.

Criterion for instability. In the absense of neutrino diffusion the hydrodynamic stability in the neutrino trapping regime of the PNS is tested by the Ledoux criterion,

$C_{\mathrm{L}} \equiv\left(\frac{\partial \rho}{\partial s}\right)_{Y, P} \frac{\mathrm{d} s}{\mathrm{~d} r}+\left(\frac{\partial \rho}{\partial Y}\right)_{s, P} \frac{\mathrm{d} Y}{\mathrm{~d} r}$

with $s=s+s_{\nu}$ being the entropy including the neutrino entropy $s_{v}$, and $Y=Y_{\text {lep }}=Y_{\mathrm{e}}+Y_{v}$ being the total lepton number. Stability means $C_{\mathrm{L}}<0$. For $2 \mathrm{D}$ models Eq. (21) is evaluated on a spherically symmetric image of the star as defined in Sect. 2.2.2. The corresponding Brunt-Väisälä frequency

$\omega_{\mathrm{BV}} \equiv \operatorname{sign}\left(C_{\mathrm{L}}\right) \sqrt{-\frac{g}{\rho}\left|C_{\mathrm{L}}\right|}$

where $g=-\mathrm{d} \Phi / \mathrm{d} r$ is the gravitational acceleration, is closely related to $C_{\mathrm{L}}$ and denotes the growth rate of fluctuations, if positive (unstable modes), and the negative of the oscillation frequency, if negative (stable modes).

Recently, Bruenn et al. (2004) presented a more elaborate discussion of hydrodynamic stability in the PNS including the effects of neutrino diffusion (an extension of a previous analysis by Bruenn \& Dineva 1996). They argue that local perturbations in the lepton number will be reflected in the neutrino phase space and thus cause a net neutrino diffusion which tries to wash out the perturbation, an effect which can be accounted for by a "response function". Since neutrinos also carry entropy the neutrino diffusion that smoothes the lepton number perturbation will create an entropy perturbation. This effect is characterized by a "cross response function". Of course, entropy perturbations will analogously induce an equilibrating net neutrino diffusion which at the same time carries lepton number, corresponding to another "response function" and a "cross response function". Bruenn et al. (2004) found in a numerical analysis that perturbation-induced neutrino diffusion transports lepton number more efficiently than entropy, and that the transport of lepton number reacts faster to entropy perturbations than to lepton number perturbations. For such a situation convective instability should set in at most stellar conditions, even when the fluid is Ledoux stable. In particular, Bruenn et al. (2004) describe two kinds of instabilities in the presence of neutrino diffusion, "lepto-entropy finger" (LEF) convection and "leptoentropy semi-convection" (LESC).

Unfortunately, Bruenn et al. (2004) did not provide detailed information about the values of the (cross) response functions in the different regions of the PNS, and the calculation of these response functions appears to be quite involved. Although one might use their approximate values for the response functions as an estimate, Bruenn et al. (2004) show that these depend 
on which interaction rates are taken into account. They further mention that the existence of LESC is sensitive to the exact values of the cross response functions. Due to these uncertainties we refrain from applying their stability analysis here. Instead, in order to account for efficient transport of lepton number happening in convective 2D models between buoyant fluid elements and their surroundings, we extend Eq. (21) to a "QuasiLedoux" criterion, following Wilson \& Mayle (1993) and Keil (1997):

$C_{\mathrm{QL}} \equiv\left(\frac{\partial \rho}{\partial s}\right)_{\langle Y\rangle,\langle P\rangle} \frac{\mathrm{d}\langle s\rangle}{\mathrm{d} r}+\left(\frac{\partial \rho}{\partial Y}\right)_{\langle s\rangle,\langle P\rangle}\left(\frac{\mathrm{d}\langle Y\rangle}{\mathrm{d} r}-\beta \frac{\mathrm{d} Y}{\mathrm{~d} r}\right)$.

Here, the brackets \langle\rangle denote angular averaging to emphasize that the last term, $\mathrm{d} Y / \mathrm{d} r$, is evaluated locally. The additional parameter $\beta$ has been introduced as a measure of the efficiency of the exchange of lepton number between a perturbed fluid element and its surroundings. From simulations it was found that values of $\beta \simeq 0.5-1.5$ are typical, and that the criterion is not very sensitive to the exact value of $\beta$ (Keil 1997). Therefore, we will use $\beta=1$.

Different from convection inside the PNS (i.e. below the neutrinosphere) exchange of entropy and lepton number by neutrino transport between moving fluid elements and their surroundings does not play an important role in the gain layer, where neutrinos and stellar fluid are much less tightly coupled. Therefore we apply Eq. (21) using the medium entropy $s$ and $Y=Y_{\mathrm{e}}$ when evaluating the stability criterion, or the BruntVäisälä frequency, at densities lower than $10^{12} \mathrm{~g} \mathrm{~cm}^{-3}$, considering the latter as the density below which neutrinos begin decoupling.

Stability analysis. In order to assess the importance of the lateral gradient terms which we take into account in our neutrinohydrodynamics treatment, we performed three test calculations which were started by imposing random seed perturbations in the density with a maximum amplitude of $\pm 2.5 \%$ on an early post-bounce model which was taken from a spherically symmetric simulation. The perturbed model was then evolved for a few milliseconds (corresponding to a multiple of the relevant dynamical timescale) in order to test different variants of the 2D transport code described above.

Figure 2 a shows the standard deviation of density fluctuations in lateral direction,

$\sigma_{\rho}(r) \equiv \sqrt{\frac{1}{N} \sum_{k} \Delta \mathrm{c} \vartheta_{k} \cdot\left[\frac{\rho_{k}(r)-\langle\rho(r)\rangle_{\vartheta}}{\langle\rho(r)\rangle_{\vartheta}}\right]^{2}}$,

where $\Delta \mathrm{c} \vartheta_{k} \equiv \cos \left(\vartheta_{k-\frac{1}{2}}\right)-\cos \left(\vartheta_{k+\frac{1}{2}}\right), N \equiv \sum_{k^{\prime}} \Delta \mathrm{c} \vartheta_{k^{\prime}}$, and $\langle\rho(r)\rangle_{\vartheta} \equiv \frac{1}{N} \sum_{k^{\prime}} \rho_{k^{\prime}}(r) \Delta \mathrm{c} \vartheta_{k^{\prime}}$, as a function of radius after $3.6 \mathrm{~ms}$ of $2 \mathrm{D}$ evolution. The quantity $\sigma_{\rho}$ serves as a convenient measure to specify the magnitude of the density fluctuations. Employing the full implementation of our neutrino treatment, which takes into account both lateral gradients in the neutrino moments equations as well as lateral gradients of the neutrino pressure in the fluid equations, the initial perturbations do not grow to large instabilities anywhere inside the PNS, which is in accordance with the prediction by our Quasi-Ledoux criterion. The residual fluctuations which can be discerned at radii

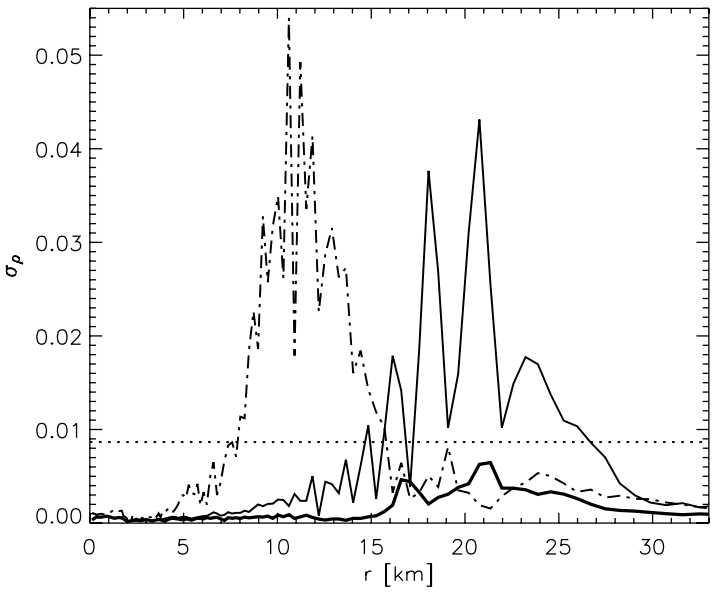

a

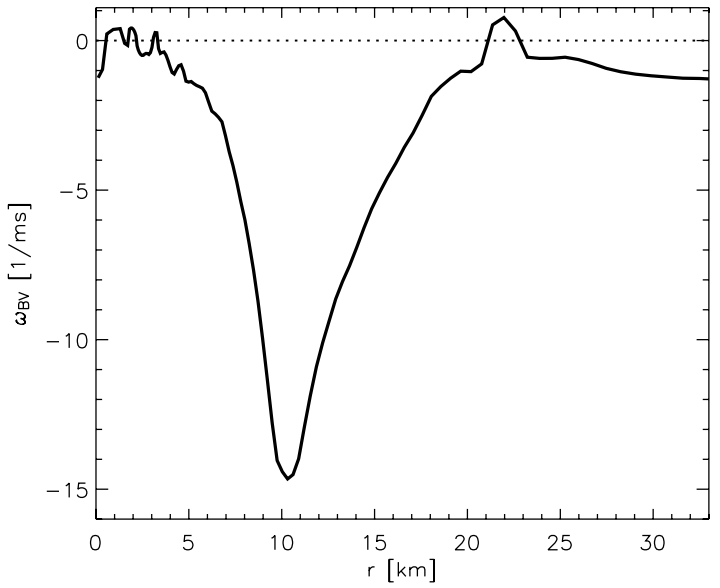

Fig. 2. a) Standard deviation of the density $\sigma_{\rho}$ (see Eq. (24)) indicating convective activity inside the neutron star. The 1D Model s15Gio_1d.b was mapped to $2 \mathrm{D}$ (16 zones with a resolution of $\left.2.7^{\circ}\right) 27 \mathrm{~ms}$ after core bounce and the density distribution $\rho$ was perturbed. The plot shows the situation after $3.6 \mathrm{~ms}$ of dynamical evolution computed with different implementations of the 2D transport equations. The dotted line is the initial value of $\sigma_{\rho}$, the thick solid line shows the standard deviation $\sigma_{\rho}$ when the lateral terms are included in our scheme as described in Sects. 2.2.1 and 2.2.3. For comparison, the thin solid line shows $\sigma_{\rho}$ when running with pure ray-by-ray transport (i.e., without all boldface terms in Eqs. (B.13)-(B.16) and without the lateral component of neutrino pressure gradients), and the dash-dotted line corresponds to a simulation where the lateral terms of Sect. 2.2.1 and Appendix B were taken into account but not the neutrino momentum transfer to the fluid discussed in Sect. 2.2.3. b) Brunt-Väisälä frequency for the same model at the beginning of the test calculations, derived from the Quasi-Ledoux criterion, Eq. (23), using $s=s+s_{v}$ and $Y=Y_{\text {lep. }}$. Negative $\omega_{\text {BV }}$ indicates convectively stable regions.

between $15 \mathrm{~km}$ and $27 \mathrm{~km}$ seem to be of physical origin because the Quasi-Ledoux criterion predicts instability in part of this region, see Fig. $2 b$.

When switching off the effects of the lateral component of the neutrino pressure gradient we notice a strong amplification of the initial perturbations in a region where the damping should be strongest according to the Quasi-Ledoux criterion. A naive ray-by-ray scheme, which in addition to lateral neutrino pressure differences also disregards the lateral gradients in the neutrino moments equations (including those terms 
which account for the lateral advection of neutrinos), produces spurious convective activity in a broad region between $15 \mathrm{~km}$ and $25 \mathrm{~km}$. The latter phenomenon is not Ledoux convection because it transports lepton number and entropy in the wrong direction (i.e. against the gradients). While lateral fluid motions (adequately modelled by the two-dimensional hydrodynamics scheme) tend to damp fluctuations, the resulting advection of electron number produces artificial flucuations of lepton number and therefore obviously instigates buoyancy motions, if the advection of electrons is not accompanied by the corresponding advection of neutrinos (i.e. if the total lepton number is not preserved in the moving fluid elements even when diffusion of neutrinos is unimportant). This is the case when the terms accounting for lateral advection in the neutrino moments equations are neglected. Neutrino pressure differences, on the other hand, produce restoring forces which help damping lateral motions after local fluctuations have been reduced by fluid advection.

We have not attempted to perform a very detailed analysis of all effects of lateral advection and pressure gradients of neutrinos to elaborate our understanding beyond the more qualitative insights described above. However, we interprete our tests as a demonstration that approximations of multi-dimensional transport schemes must be tested carefully for the possibility of producing spurious convective activity (or suppression of convection) in the newborn neutron star, where neutrinos contribute significantly to the total pressure and total lepton number density.

\subsubsection{Lateral propagation of neutrinos}

A number of our two-dimensional supernova simulations, in particular those which produced lively hot-bubble convection, showed transient neutrino-bursts when narrow downflows of accreted stellar gas entered the cooling region and penetrated down to the vicinity of the neutrinosphere. In our simulations such bursts occurred roughly every $20 \mathrm{~ms}$, typically persisting for a few ms. The neutrinos of such bursts, which are the result of locally enhanced neutrino emission, would naturally propagate in all directions in a fully multi-dimensional treatment and would therefore illuminate the surroundings in all directions. Our code ignores the lateral propagation of these neutrinos, thus the burst is radiated away in the radially outward direction and its lateral width is essentially constrained to the layers above the hot, radiating area. Here we shall argue that although locally and transiently the neutrino heating rates can be incorrect by up to a factor of two (in very rare, extreme cases) due to the disregard of lateral neutrino propagation, the transfer of energy between neutrinos and stellar gas is not significantly changed on larger spatial and temporal scales, and therefore the global dynamics of our supernova simulations is not likely to be affected significantly.

Basically, our simplified neutrino transport overestimates the heating in the radial direction at polar latitudes where "hot spots" occur, while adjacent "rays" experience less heating than in a $2 \mathrm{D}$ neutrino transport which includes lateral neutrino fluxes. Truly two-dimensional transport tends to redistribute neutrinos in lateral direction, in particular in the semi-transparent and transparent regimes where the mean free path becomes large. This can lead to a more uniform spatial distribution of neutrinos exterior to the neutrinosphere than in case of our ray-by-ray treatment, in particular in the presence of local hot spots (see also the discussion in Livne et al. 2004).

To test the implications of such a lateral neutrino redistribution for the neutrino heating behind the shock, we performed a post-processing analysis in which we averaged the frequencydependent neutrino densities over all polar angles at a given radius and time and used the result to recalculate the local net heating rates. Then we compared the recalculated heating rate with the actual heating rate of the simulation, both integrated over the respective gain layer, for different times. Hereby the gain layer was defined for each grid value of $\vartheta$ separately as the region between the shock and the gain radius, i.e. the innermost radial point where net heating occurs. For this purpose, the position of the gain radius was also redetermined for the recalculated heating rates.

We have carried out this analysis for an $11.2 M_{\odot}$ progenitor (s11.2, Woosley et al. 2002) computed with 32 angular zones and $\sim 90^{\circ}$ lateral wedge during its post-bounce evolution (Buras et al. 2003b), a model which showed powerful hot bubble convection. The evaluation was started at $t_{\mathrm{pb}}=100 \mathrm{~ms}$; before that time hot bubble convection was weak and no strong local bursts of accretion luminosity happened. We found that the angular averaging of the neutrino densities hardly changes the total net heating rate in the gain layer, $\delta_{t} E_{\mathrm{gl}}$, see Fig. 3, upper panel. At very few instants of evaluation we discovered a significant increase of $\delta_{t} E_{\mathrm{gl}}$ by at most $30 \%$. In the temporal average this difference is reduced to only a few percent. For the average net heating per baryon in the gain layer (Fig. 3, middle panel), which, concerning global supernova dynamics, we consider to be the more decisive quantity (Janka 2001), the values for the two heating rates are almost indistinguishable.

Interestingly, the differences are somewhat larger if we separately analyze downflows of cold material and hot bubbles in the gain layer; a zone of the numerical grid is attributed to a downflow if the negative velocity is more than 1.5 times the angle-averaged (negative) velocity $\langle v\rangle_{\vartheta}$ at a given radius $r$, otherwise we define the zone to belong to a high-entropy bubble. For the downflows (see also Fig. 3, upper panel, lower curve), the time-averaged total net heating decreases by $14 \%$ in case of the angle-averaged neutrino density. On the other hand it increases by $12 \%$ for the high-entropy bubbles.

The results from this analysis should be read carefully: it does not take into account the dynamical consequences of the altered heating rates. Also, the angle-averaging of the neutrino density overestimates the spreading of the burst neutrinos in all directions, especially close to the gain radius, where we obtain by far most of the heating. The nevertheless modest sensitivity of the integral quantities to the described averaging of the neutrino distribution, however, gives us some confidence that our approximation of the moments equations is a reasonable step towards fully consistent multidimensional simulations.

Even more difficult to assess quantitatively is the effect of replacing the Eddington tensors (cf. Appendix B) by scalar variable Eddington factors which are calculated from a 


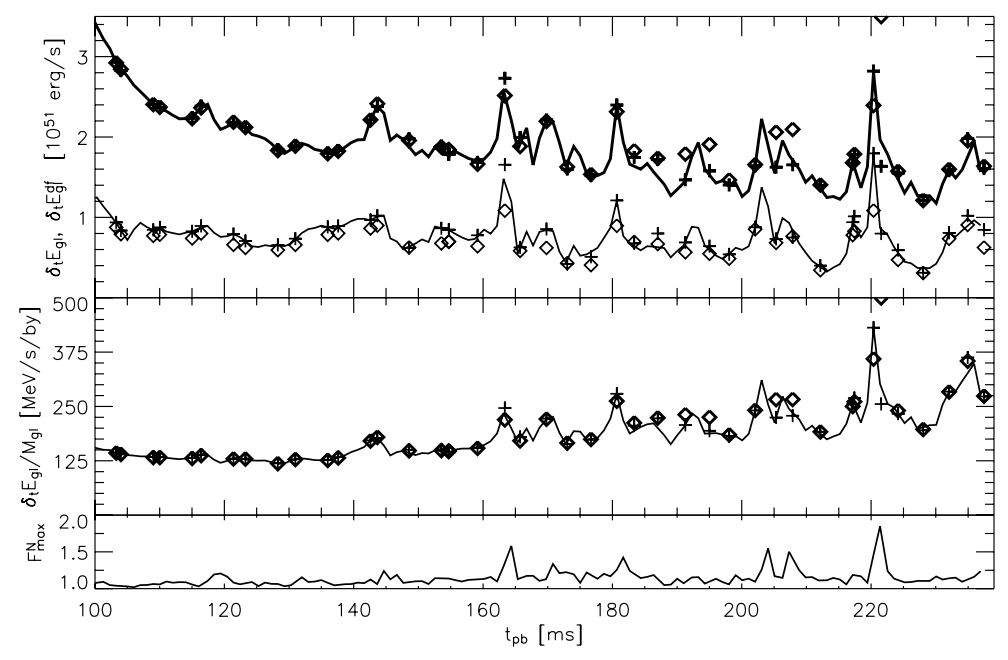

Fig. 3. The upper panel compares total (volume integrated) neutrino heating rates in the gain layer $\delta_{t} E_{\mathrm{gl}}$. The bold upper solid line shows the evolution of $\delta_{t} E_{\mathrm{gl}}$ for the $11.2 M_{\odot}$ supernova model of Buras et al. (2003b). The post-processed heating rate obtained by averaging the neutrino densities over all latitudes is represented by diamond-like symbols. For a consistency check crosses are drawn for the postprocessed neutrino heating rate without using the lateral averages of the specific neutrino density. Ideally the latter postprocessed results should be equal to the values returned from the model run. Remaining differences result from the fact that the post-processing may yield a gain radius shifted by one radial zone. For drawing the second curve (thin solid line) and the corresponding symbols the analysis was restricted to downflows in the gain layer, i.e. for regions with velocity $v<1.5\langle v\rangle_{\vartheta}<0$. The middle panel shows the average net heating rate per baryon (the symbols have the same meaning as in the upper panel), i.e. the total neutrino heating rate in the gain layer divided by the total mass $M_{\mathrm{gl}}$ contained in this region. The lower panel shows the maximum neutrino flux as measured slightly above the gain radius normalized to the flux average over all latitudes at the same radius, $F_{\max }^{\mathrm{N}}=\max _{\vartheta}(F) /\langle F\rangle_{\vartheta}$. The values indicate the relative strength of localized luminosity outbursts. solution of the one-dimensional transport equations using a spherically symmetric image of the star. We have to rely on the fact that these variables are normalized moments of the neutrino phase space distribution and thus, in the absence of persistent global deformation of the star, should not show significant variation with the angular coordinate (cf. Rampp 2000; Rampp \& Janka 2002). Anyway, as we have seen, ignoring the lateral propagation of neutrinos does not seem to largely affect the neutrino-matter coupling in the semi-transparent region. Further, Thompson et al. (2005) have recently shown that neutrino viscocity, which is connected with the non-diagonal elements of the Eddington tensors, is of minor importance. Therefore we hope that the transport is also sufficiently insensitive to our approximation concerning the variable Eddington factors. Final answers can only be expected from simulations employing a fully multidimensional treatment of the neutrino transport.

\subsection{Equation of state}

All calculations presented in this paper were run with the equation of state (EoS) described in detail in Rampp \& Janka (2002, Appendix B). The EoS of Lattimer \& Swesty (1991) (LS EoS) is employed to treat matter at densities above $6 \times 10^{7} \mathrm{~g} \mathrm{~cm}^{-3}$. Recently it became clear that this EoS underestimates the fraction of alpha particles (due to an error in the definition of the alpha particle rest mass; Lattimer, personal communication) and concerns were expressed that deficiencies of this EoS in the density regime between $10^{8} \mathrm{~g} \mathrm{~cm}^{-3}$ and $10^{11} \mathrm{~g} \mathrm{~cm}^{-3}$ could have consequences for the possibility of an explosion, which we fail to get with the LS EoS (Fryer, personal communication).

In order to test this we have implemented a new EoS in our code. This EoS is similar to the low-density EoS (Janka 1999) described by Rampp \& Janka (2002, Appendix B), but the very approximative handling of nuclear dissociation and recombination in nuclear statistical equilibrium (NSE) in that EoS is now replaced by a composition table in the $\left(\rho, T, Y_{\mathrm{e}}\right)$-space. This table was computed assuming matter to be in NSE and solving the Saha equations for equilibrium of a mixture of free neutrons and protons, ${ }^{4} \mathrm{He}$, and ${ }^{54} \mathrm{Mn}$ as a representative heavy nucleus (Janka 1991). We use the table for $T>5 \times 10^{9} \mathrm{~K}$, where NSE is a reasonably good assumption. Given the nuclear composition, variables such as pressure and entropy can be calculated from the EoS of Janka (1999).

A comparison of this simple 4-species NSE table with a sophisticated NSE solver that takes into account 32 different species of heavy nuclei showed excellent agreement in entropy, pressure, and helium mass fraction for typical conditions met in the post-shock layer. Note that the 4-species table overestimates the mass fraction of free nucleons in parameter regions where heavy nuclei dominate the composition and $Y_{\mathrm{e}}$ is far from $25 / 54 \simeq 0.463$ (e.g. for $T=1 \mathrm{MeV}, Y_{\mathrm{e}}=0.3$ this is the case at $\rho>3 \times 10^{10} \mathrm{~g} \mathrm{~cm}^{-3}$, see Fig. 4). This problem originates from the restriction to one fixed, representative heavy nucleus and the necessity to fulfill charge neutrality. However, NSE in the post-shock layer of a supernova is characterized by conditions where essentially all heavy nuclei are dissociated into free neutrons, protons, and $\alpha$-particles.

Comparing our composition table with data from the LS EoS (Fig. 4) we indeed observe a significantly larger helium mass fraction for certain combinations of density and temperature. Moreover, the region where helium contributes significantly to the composition is larger. This also affects the pressure and entropy, especially at low temperatures and entropies (Fig. 4).

To test whether the sizeable differences between the EoS using our NSE table at densities below $10^{11} \mathrm{~g} \mathrm{~cm}^{-3}$ and the LS EoS have any influence on supernova simulations we ran the 
R. Buras et al.: Two-dimensional hydrodynamic core-collapse supernova simulations with spectral neutrino transport. I.

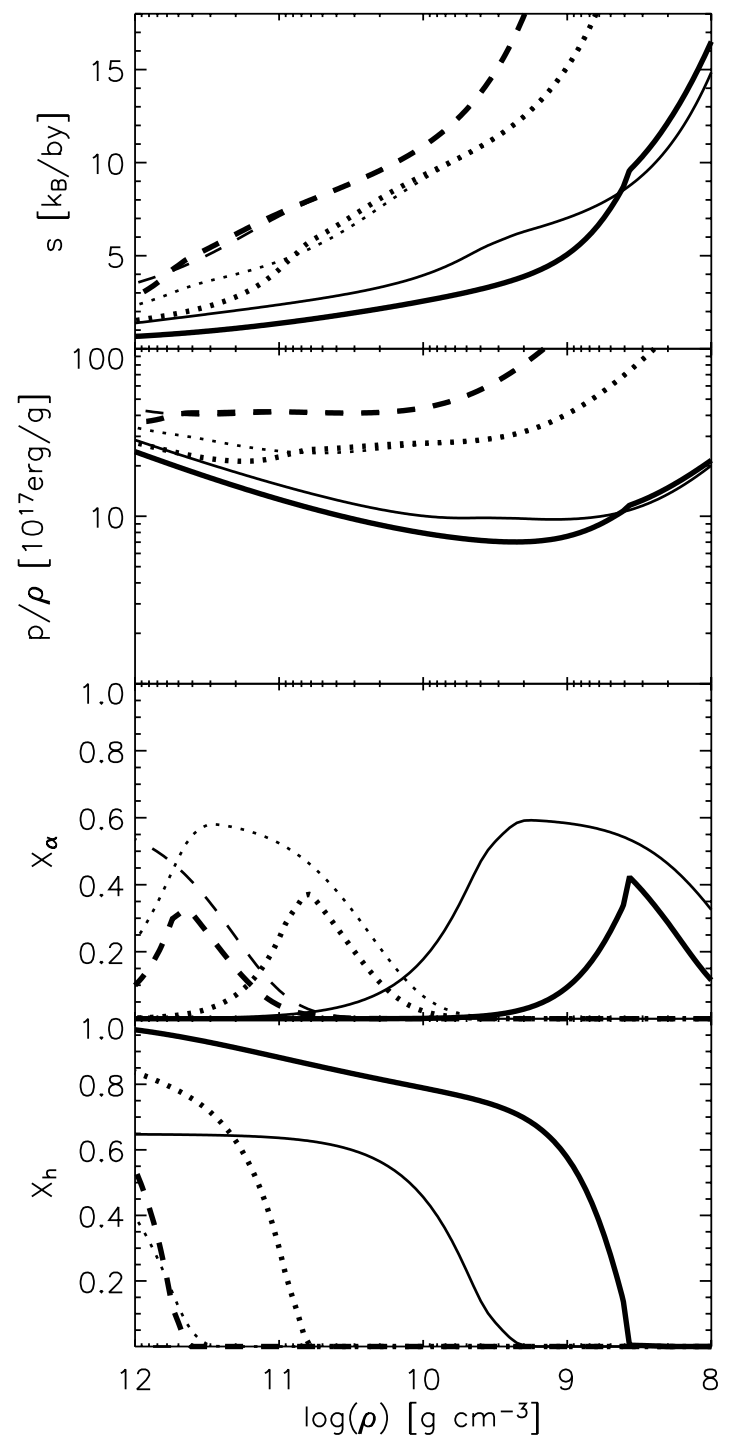

Fig. 4. Comparison of the LS EoS (thick lines) with the four-species NSE table introduced here (thin lines), for $Y_{\mathrm{e}}=0.3$ and $T=1$ (solid), 2 (dotted), and 3 (dashed) MeV. $X_{\alpha}$ and $X_{\mathrm{h}}$ are the mass fractions of $\alpha$-particles and of the representative heavy nucleus, respectively.

spherically symmetric $11.2 M_{\odot}$ model of Buras et al. (2003b) with both equations of state. This comparison directly tests the influence of the different EoS implementations on the dynamical evolution of the supernova.

Surprisingly, both runs showed virtually no difference in the post-bounce history of the supernova (Fig. 5) and only small differences in the post-shock structure (Figs. 6 and 7), even though a post-processing analysis where values of $T, \rho$, $Y_{\mathrm{e}}$ were crosswise fed into the two variants of the EoS revealed differences in entropy and pressure of up to $15 \%$ and $10 \%$, respectively, below the shock (Fig. 8). This phenomenon has a simple explanation: on the one hand the relatively high entropies behind the shock in our models imply that the influence of $\alpha$-particles is in general moderate (Fig. 4). On the other hand the accretion layer behind the supernova shock can be considered as an approximately hydrostatic situation where the velocities of the shocked medium and of the shock itself are much

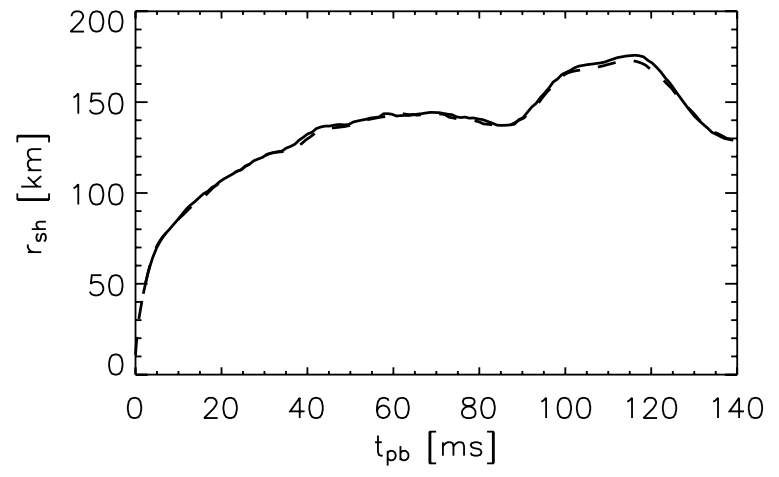

Fig. 5. Shock positions for the two dynamical simulations of the postbounce evolution of an 11.2 $M_{\odot}$ star with the LS EoS (dashed) and the four-species NSE table (solid) used in the density regime below $10^{11} \mathrm{~g} \mathrm{~cm}^{-3}$.

smaller than the sound speed $c_{\mathrm{s}}$. In this case the pressure profile behind the shock is determined by constraining boundary conditions, i.e. the mass and radius of the proto neutron star (and therefore its gravitational field) on the one hand, and the mass accretion rate at the shock on the other (Janka 2001). Keeping in mind that the Rankine-Hugoniot conditions connect the flow in the infall region with the conditions in the post-shock layer, the pressure profile in the accretion layer is fully determined.

Thus, for a given pressure profile other variables, such as $\rho$ and $T$, may vary for different EoSs (see Fig. 7) without changing the dynamical evolution of the supernova. Only if these variables have an immediate influence on the evolution, e.g. by temperature-dependent neutrino emission, do we have to worry about the differences and consequences of the composition. Neutrino heating, however, is dynamically not very important in the gain layer of our non-exploding models, while in the cooling region the composition differences between the EoSs are negligibly small.

We conclude that, although the LS EoS has problems with the $\alpha$-mass fraction and other dependent variables in the lowdensity regime at supernova conditions, we do not find a major effect on the dynamics of our simulations.

\subsection{Entropy wiggles}

All the models presented here feature a numerical problem, the origin of which we found only recently. In all profiles of 1D calculations, and also partly in 2D calculations (when convective effects did not wash out the effect), artificial wiggles appear behind the shock. These wiggles are most pronounced in the entropy and pressure profiles with amplitudes of up to $10 \%$, see e.g. Fig. 8.

The problem is connected with the use of the specific en$\operatorname{ergy} \varepsilon=e+e_{\text {kin }}$ in the hydrodynamics Eqs. (1)-(7), which are solved in the PROMETHEUS code with a Riemann solver technique, see Sect. 2.1, and the corresponding calculation of the required index $\widetilde{\Gamma}_{e} \equiv \frac{p}{e \rho}+1$ with an arbitrarily normalized energy $e$ instead of the internal energy $e_{\text {int }}$. The Riemann solver expects $\widetilde{\Gamma}_{e}$ to be calculated with the internal energy $e_{\text {int }}$, because for thermodynamical consistency between $\widetilde{\Gamma}_{e}$ and the adiabatic index $\Gamma_{\mathrm{ad}}=(\mathrm{d} \ln p / \mathrm{d} \ln \rho)_{s}$, the energy $e$ and the 


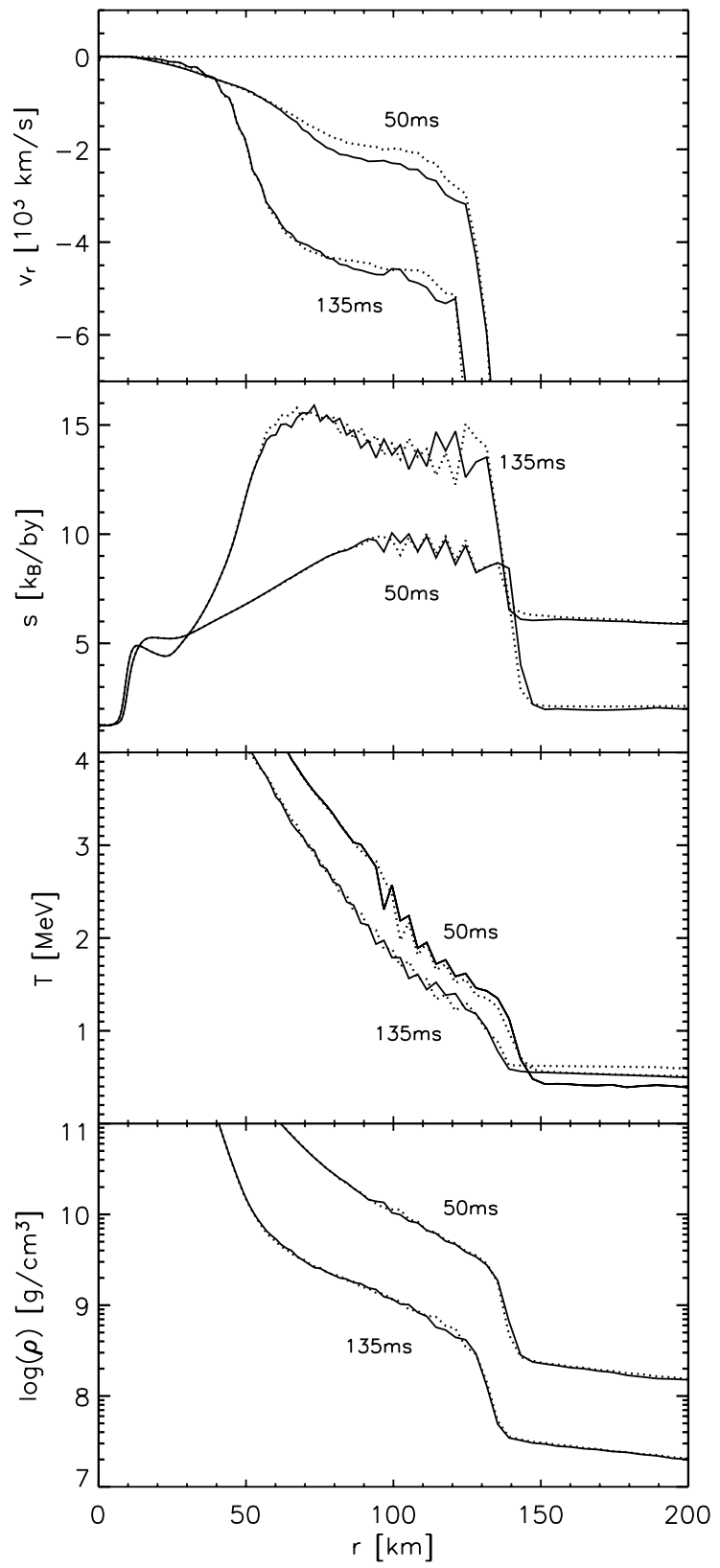

Fig. 6. Comparison of the profiles for the two dynamical simulations of the post-bounce evolution of an 11.2 $M_{\odot}$ star with the LS EoS (dotted) and the four-species NSE table (solid) used in the density regime below $10^{11} \mathrm{~g} \mathrm{~cm}^{-3}$ at two post-bounce times.

pressure $p$ should go to zero simultaneously. On the other hand, the physics should only depend on energy differences, and the energy normalization should not play a role, i.e., the EoS as well as the hydrodynamics should remain unchanged, independent of the chosen zero point of the energy. Therefore it is allowed to add an arbitrary normalization to $e$, still retaining the validity of Eqs. (1)-(7). In our code, we use the definition

$e=e_{\text {int }}+e_{\mathrm{rm}}+e_{0}$,

where $e_{\mathrm{rm}}$ is the specific baryon and electron rest mass energy and $e_{0}$ is a constant offset chosen to be in accordance with the energy definition in our high-density EoS provided by Lattimer \& Swesty (1991).

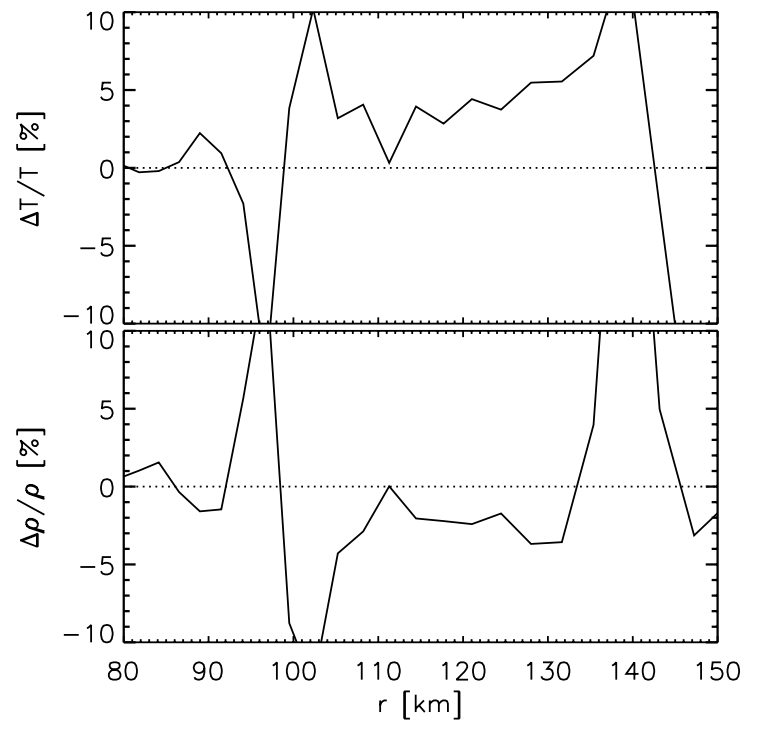

Fig. 7. Relative difference of temperature and density between the simulations described in Fig. 6 at $t=50$ ms post-bounce. $\Delta T>0$ means higher $T$ in the simulation with the four-species NSE table (solid line in Fig. 6) used in the density regime below $10^{11} \mathrm{~g} \mathrm{~cm}^{-3}$, dito for $\Delta \rho$.

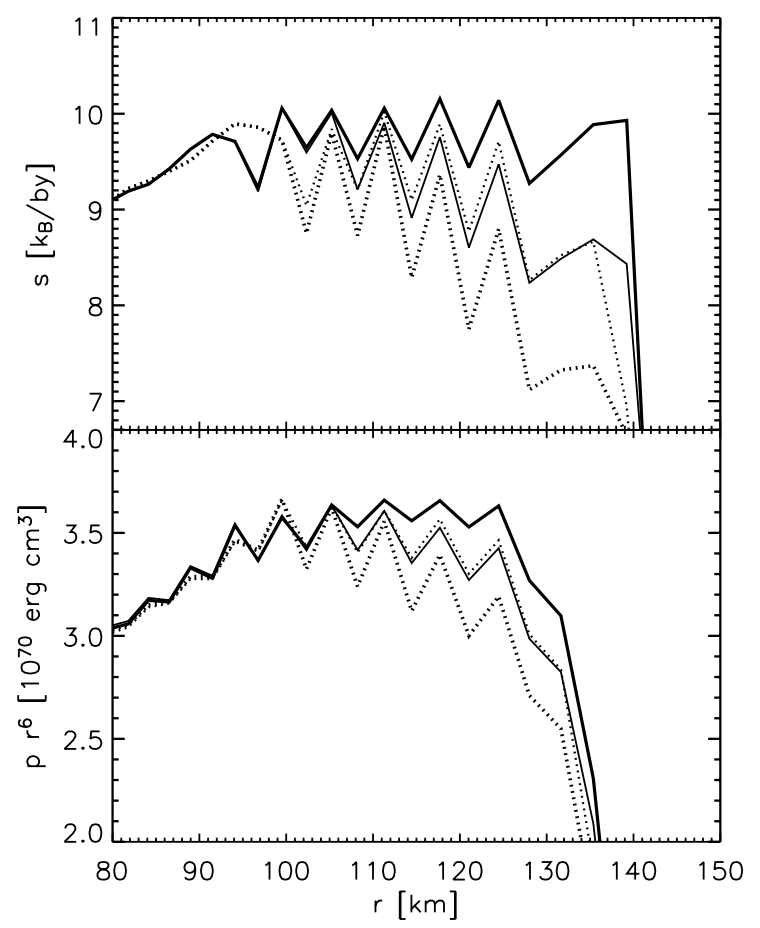

Fig. 8. Enlarged comparison of the entropy and the pressure (renormalized with $r^{6}$ ) between the two simulations shown in Fig. 6 at $t=50 \mathrm{~ms}$. The thin lines correspond to the actual values, the thick lines correspond to a post-processing analysis of the profiles using the respectively other variant of the EoS.

$\widetilde{\Gamma}_{e}$ as defined above is therefore different from the thermodynamical index $\Gamma_{e}=\frac{p}{e_{\text {int } \rho}}+1$, which is expected to be fed into the Riemann solver. In particular, in regions with relatively low internal energy and large value of $\left(e_{\mathrm{rm}}+e_{0}\right)$ (e.g., just behind the shock where the matter consists mostly of free nucleons), $\widetilde{\Gamma}_{e}$ with our chosen normalization of $e$ can have values which are significantly below the physically meaningful lower bound 
$\mathbf{a}$

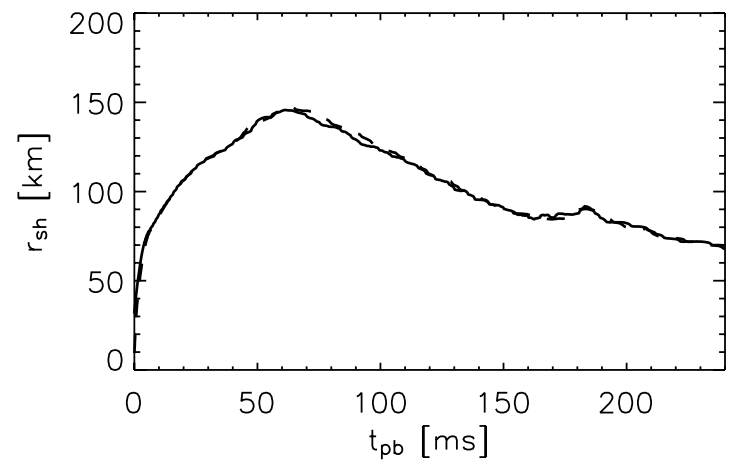

b

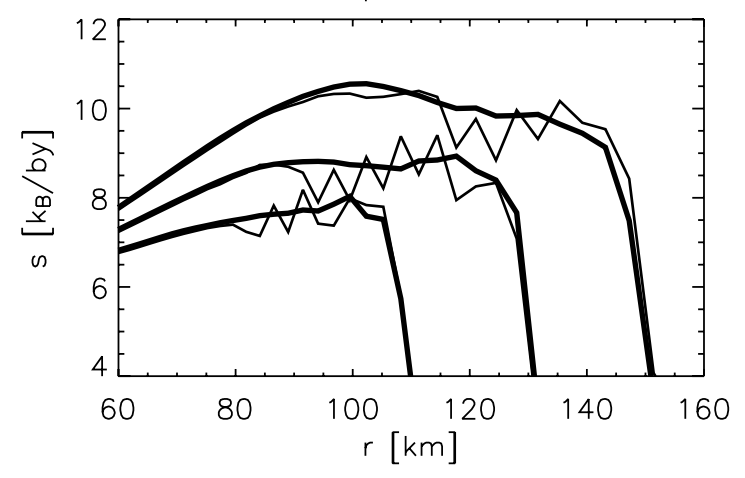

Fig. 9. a) Shock trajectories of Model s15Gio_1d.b, described in Sect. 3.1.1 (solid), and a calculation with identical micro-physics but with our new definition of the energy $e$ (dashed). Note that the visible very small differences actually originate from slight differences of the gravitational potentials used in both simulations. b) Entropy profiles for Model s15Gio_1d.b (thin) and the simulation with our new definition of the energy $e$ (thick) at the times $20 \mathrm{~ms}, 40 \mathrm{~ms}$, and $60 \mathrm{~ms}$ after bounce (from bottom to top).

of $4 / 3$. Empirically, we found that the numerical noise appears whenever $\widetilde{\Gamma}_{e}<4 / 3$, which turned out to be the case in the post-shock region. However, we did not make efforts to locate the source of these numerical fluctuations more precisely than linking it to the use of a Riemann solver technique in cases where $\widetilde{\Gamma}_{e}$ adopts unphysical values.

We have solved this problem by renormalizing the energy used in the integration scheme of the hydrodynamics equations relative to $e$ by subtracting from $e$ the additional terms $e_{\mathrm{rm}}+e_{0}$ of Eq. (25). The numerical procedure is described in detail in Appendix C. In a test calculation with this improved handling, the wiggles turned out to disappear, see Fig. 9b.

Figure 9 also shows that the dynamics and global evolution of the models are not affected by the wiggles. This confirms that the hydrodynamics solutions given by PROMETHEUS are not globally flawed even if $e$ from Eq. (25) and $e_{\text {int }}$ deviate significantly from each other. Therefore the simulations presented in this paper are sound despite the wiggles in the postshock profiles (see also Liebendörfer et al. 2005 for a confirmation). Of course, future calculations will be performed using the improved treatment of Appendix C.

\section{Results}

Here we present results from one-dimensional and from first two-dimensional simulations with our "coupled ray-by-ray" approximation of spectral neutrino transport and with an implementation of neutrino opacities which is improved compared to Bruenn (1985). First, we will discuss the differences resulting from our enlarged set of opacities in the context of spherically symmetric (1D) models. We will also discuss the influence of general relativistic corrections on the core collapse and post-bounce evolution and will investigate the importance of velocity-dependent terms in the neutrino momentum equation, especially for a quantitatively correct description of neutrino heating and cooling between proto neutron star (PNS) and supernova shock. In this context we shall present an interesting 1D model which shows largeamplitude oscillations of the shock position and neutrino luminosities. Then we will elaborate on our first two-dimensional (2D) neutrino-hydrodynamical simulation with spectral neutrino transport. Finally, we present a 2D model that develops an explosion when we retreat from the most complete version of our neutrino transport treatment by neglecting the velocitydependent terms in the neutrino momentum equation.

Progenitor and model notation: the names of our models are chosen with the aim to provide information about the employed input physics. All models in this paper were started from the progenitor "s15s7b2", a star with a main-sequence mass of $15 M_{\odot}$ kindly provided to us by Woosley (Woosley \& Weaver 1995), see Fig. 10. Thus our model names start with "s15". The simulations were performed either with Newtonian gravity ("N") or with our approximative implementation of general relativistic gravity ("G"). While most simulations included the most advanced description of neutrino interactions ("io"), we performed a number of calculations with the standard opacities of Bruenn (1985) plus nucleon-nucleon bremsstrahlung ("so"). One-dimensional models are labelled with "_1d", the names of the two-dimensional models give information about the number of lateral zones $N_{\vartheta}$ (in this paper only “_32"). Combining this with the chosen size of the angular wedge allows one to infer the equidistant angular zoning of the model. Finally, it is very important whether a model employed our full implementation of the neutrino transport, Case B (".b"), or whether the radial velocity-dependent terms (except for the advection terms) in the neutrino momentum equation were omitted, Case A (".a"). The models presented in this paper are listed in Table 1. The collapse phase was calculated with a Lagrangian grid, the post-bounce phase with a Eulerian grid, and the simulations were run with 400 radial hydro zones and 230 radial neutrino transport zones, both with geometric spacing. The number of tangent rays employed was 250 . Inside of $400 \mathrm{~km}$, the hydro and transport zones were chosen to be identical, outside of $400 \mathrm{~km}$ only a few transport zones were added to correctly simulate the neutrino propagation out to the outer boundary of the grid at $10^{4} \mathrm{~km}$. All models were run with 17 geometrically spaced neutrino energy bins between $2 \mathrm{MeV}$ and $380 \mathrm{MeV}$. When necessary, the radial grids 


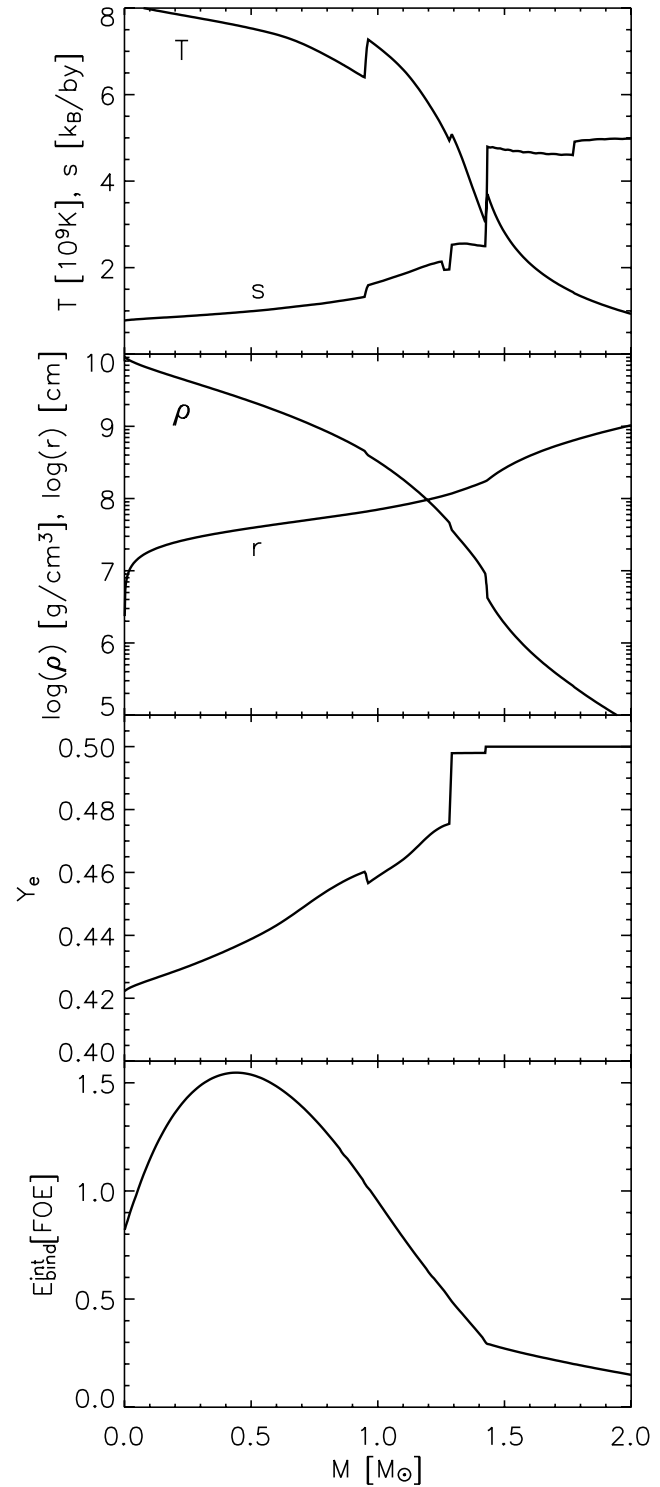

Fig. 10. Progenitor structure. Temperature $T$, entropy per baryon $s$, electron fraction $Y_{\mathrm{e}}$, density $\rho$, radius $r$, and shell binding energy $E_{\mathrm{bind}}^{\text {shell }}$, defined in Eq. (26), as functions of the enclosed mass. At $1.42 M_{\odot}$ the interface between the silicon shell and the oxygen-rich silicon shell is located. $T, Y_{\mathrm{e}}$, and $\rho$ are taken from model $\mathrm{s} 15 \mathrm{~s} 7 \mathrm{~b} 2$ of Woosley $\&$ Weaver (1995), while the $s$ and $E_{\text {bind }}^{\text {shell }}$ are computed by applying our EoS.

were refined to account for the steepening density gradient at the surface of the PNS.

Definitions: we define the shock radius $r_{\text {shock }}$ as the radial position where the radial velocity in the shock "discontinuity" is half of the minimum preshock value of the velocity. The gain radius $r_{\text {gain }}$ is the zone interface below the innermost radial zone (at given value of $v$ ) outside of which no zones with net cooling exist. Thus the gain layer is between $r_{\text {gain }}$ and $r_{\text {shock }}$ while the cooling region is below $r_{\text {gain }}$ and includes the PNS. The total net energy transfer rates between neutrinos and medium, $\delta_{t} E_{\mathrm{cool}}$ and $\delta_{t} E_{\mathrm{gl}}$ in the cooling region and gain layer are defined as integrals over the respective regions. An interesting quantity is the "shell binding energy" $E_{\text {bind }}^{\text {shell }}$, i.e. the energy needed to lift all material above a considered radius out of the gravitational potential of the mass enclosed by this radius and thus move the material to infinity. In the approximation with Newtonian 1D gravity (which is a very good assumption for the progenitor) its definition is

$$
\begin{aligned}
& E_{\text {bind }}^{\text {shell }}(r) \equiv \frac{4 \pi}{\cos \vartheta_{\max }-\cos \vartheta_{\text {min }}} \\
& \quad \times \int_{r}^{\infty} \int_{\cos \vartheta_{\text {min }}}^{\cos \vartheta_{\text {max }}} \varepsilon_{\text {bind }}^{\text {shell }}\left(r^{\prime}, \vartheta\right) \rho\left(r^{\prime}, \vartheta\right) r^{\prime 2} \mathrm{~d} \cos \vartheta \mathrm{d} r^{\prime},
\end{aligned}
$$

where $\varepsilon_{\text {bind }}^{\text {shell }}$ is our so-called "local specific binding energy",

$\varepsilon_{\text {bind }}^{\text {shell }}(r, \vartheta) \equiv e_{\text {int }}(r, \vartheta)+\frac{1}{2}\left(v_{r}^{2}+v_{\vartheta}^{2}+v_{\varphi}^{2}\right)(r, \vartheta)+\Phi_{1 \mathrm{D}}^{\text {enclosed }}(r)$.

The gravitational potential $\Phi_{1 \mathrm{D}}^{\text {enclosed }}(r)$ is calculated taking into account only the mass inside of the radius $r$ and assuming it to be distributed spherically symmetrical. These energies, as well as the neutrino luminosities, are generally given in the unit of FOE, the abbreviation for "ten to the fifty-one erg". Finally, the spectrally averaged transport optical depth is evaluated according to the formula

$$
\begin{aligned}
\tau_{v}(r) & \equiv \int_{r}^{\infty}\left\langle\kappa_{v}\right\rangle\left(r^{\prime}\right) \mathrm{d} r^{\prime} ; \\
\left\langle\kappa_{v}\right\rangle\left(r^{\prime}\right) & \equiv \int_{0}^{\infty} \lambda_{v}^{-1}\left(r^{\prime}, \epsilon\right) H_{v}\left(r^{\prime}, \epsilon\right) \mathrm{d} \epsilon \mid \int_{0}^{\infty} H_{v}\left(r^{\prime}, \epsilon\right) \mathrm{d} \epsilon,
\end{aligned}
$$

where $\lambda_{v}^{-1}\left(r^{\prime}, \epsilon\right)=\sum_{i} \lambda_{v, i}^{-1}$ is the inverse mean free path for neutrinos of energy $\epsilon$ and type $v$ including all scattering and absorption processes. The different contributions to the sum are taken from the RHS of the neutrino momentum equation as implemented in our transport code. We define an "energyaveraged" neutrinosphere (radius) by the condition $\tau_{v}\left(r_{v}\right)=1$. Where needed, the energy-dependent neutrinosphere is defined by $\tau_{v}\left(r_{v}(\epsilon), \epsilon\right)=\int_{r}^{\infty} \lambda_{v}^{-1}\left(r^{\prime}, \epsilon\right) \mathrm{d} r^{\prime} \equiv 1$. Many properties of 2D models are evaluated from 1D radial profiles, obtained by laterally averaging the $2 \mathrm{D}$ data, see Sect. 2.2.2 for the definition of this procedure. This also includes the definition of "mass shells" in $2 \mathrm{D}$, i.e. a mass shell corresponds to the radius of the sphere which encloses this mass. For 2D simulations, the criterion of convective instability (see Sect. 2.3.2) is also evaluated with laterally averaged variables.

\subsection{Spherically symmetric models}

\subsubsection{The reference model}

This one-dimensional model, s15Gio_1d.b, was run with our most elaborate "state-of-the-art" description of neutrino opacities described in Appendix A and references therein. This treatment of neutrino-matter interactions is improved compared to the rates of Bruenn (1985), which we consider as the "standard case", by including effects of nucleon recoil and thermal motions, weak magnetism corrections (Horowitz 2002), and nucleon-nucleon correlations (Burrows \& Sawyer 1998, 1999) in neutrino-nucleon interactions. Moreover, the reduction of the effective nucleon mass at nuclear densities (Reddy et al. 1999) 
Table 1. Input physics for the sample of computed models presented here.

\begin{tabular}{lllllll}
\hline \hline Model & Dim. & Gravity & $v$ Reactions & Transport & Wedge $^{a}$ & $\vartheta$-zones \\
\hline s15Nso_1d.b & 1D & Newtonian & standard & Case B & & \\
s15Gso_1d.b & 1D & approx. GR & standard & Case B & & \\
s15Gso_1d.b* & 1D & approx. GR & standard $^{b}$ & Case B & & \\
s15Gio_1d.a & 1D & approx. GR & improved $^{c}$ & Case A & & \\
s15Gio_32.a & 2D & approx. GR & improved & Case A & $\pm 43.2^{\circ}$ & 32 \\
s15Gio_1d.b & 1D & approx. GR & improved & Case B & & \\
s15Gio_32.b & 2D & approx. GR & improved & Case B & $\pm 43.2^{\circ}$ & 32 \\
\hline
\end{tabular}

${ }^{a}$ Angular wedge of the spherical coordinate grid around the equatorial plane.

${ }^{b}$ Calculation without neutrino-pair creation by nucleon-nucleon bremsstrahlung.

${ }^{c}$ Calculation without the neutrino-antineutrino processes of Buras et al. (2003a).

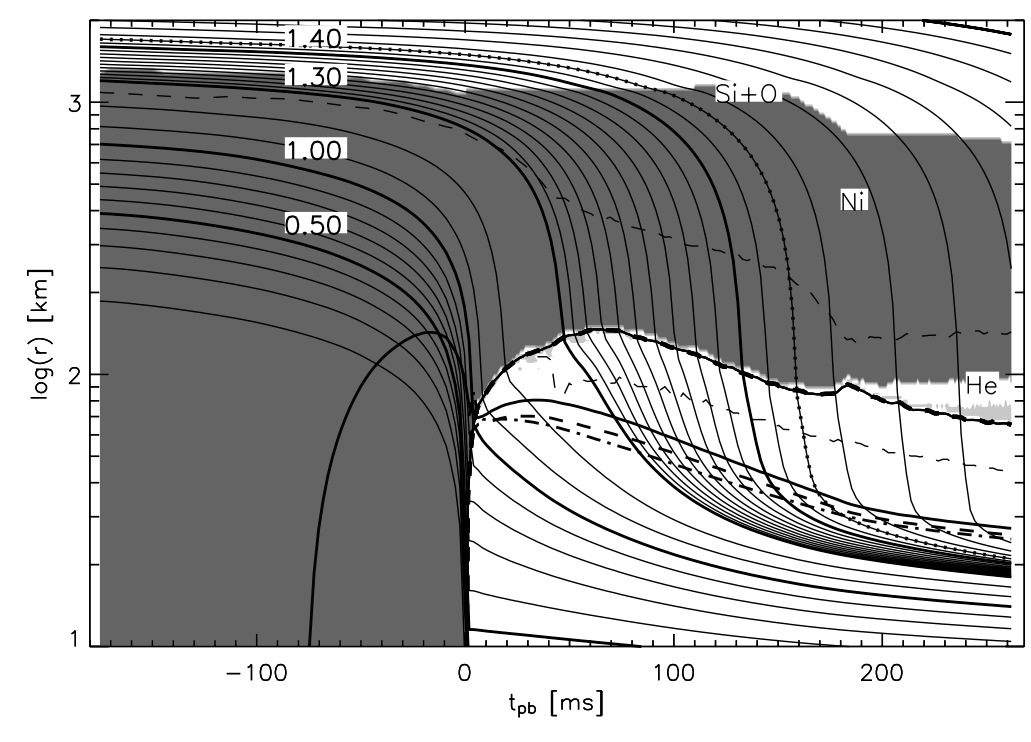

Fig. 11. Trajectories of mass shells for Model s15Gio_1d.b. The plot also shows the neutrinospheres for $v_{\mathrm{e}}$ (thick solid line), $\bar{v}_{\mathrm{e}}$ (thick dashed), and $v_{\mathrm{x}}$ (thick dash-dotted), the mass shell at which the silicon shell becomes oxygen-rich (knotted solid line, at $1.42 M_{\odot}$ ), and the shock (thick solid line with superimposed dashes). Further we have marked the regions with a mass fraction of more than $60 \%$ in iron-group elements (dark shaded). Also shown are regions with a mass fraction between $30 \%$ and $60 \%$ in ${ }^{4} \mathrm{He}$ (light shaded). Note that the white region at $t_{\mathrm{pb}}>200 \mathrm{~ms}$ and $r \sim 90 \mathrm{~km}$ corresponds to $X_{\mathrm{Fe}}<0.6$ and $X_{\mathrm{He}}<0.3$. Finally, the lower thin dashed line marks the gain radius, while the upper one marks the interface between our high-density and low-density EoSs (i.e. the upper thin dashed line corresponds to a density of $\left.\rho=6 \times 10^{7} \mathrm{~g} \mathrm{~cm}^{-3}\right)$. and the quenching of the axial-vector coupling in nuclear matter (Carter \& Prakash 2002) are taken into account. In addition, we include nucleon-nucleon bremsstrahlung and neutrinoneutrino interactions like scattering as well as pair creation processes between neutrinos of different flavors. The full list of included rates and corresponding references can be found in Table A.1.

We applied the approximations of general relativity described in Sects. 2.1 and 2.3.1 (see also Rampp \& Janka 2002; and Marek et al. 2006). Finally, the simulation was done including the velocity-dependent terms in the neutrino momentum equation, i.e. the terms proportional to $\beta_{r}$ in Eqs. (B.14), (B.16). Despite of being formally small $\left(\beta_{r} \leq 0.1\right)$ these terms turn out to be important in supernova simulations as we will elaborate in Sect. 3.1.3.

The evolution of this model can be seen in Fig. 11. The progenitor needs $174 \mathrm{~ms}$ to reach core bounce. The typical time evolution of the central values of $Y_{\text {lep }}$ and $s$ during collapse can be seen as functions of the central density in Fig. 12. The shock is created at an enclosed mass of $M_{\mathrm{sc}}=0.49 M_{\odot}$ and a radius of $r_{\mathrm{sc}}=10.6 \mathrm{~km}$. Its prompt expansion stalls after about $1 \mathrm{~ms}$ at $r=32 \mathrm{~km}$, turning the shock into an accretion front. Initially the high mass accretion rate through the shock leads to matter being accumulated between the PNS and the shock; the energy via neutrino cooling is not quick enough to allow this material to settle quickly so that the shock continues to move outward. After $6 \mathrm{~ms}$, when the shock reaches $80 \mathrm{~km}$, this expansion slows down because the mass accretion rate drops and neutrino cooling has become more efficient, leading to an "equilibrium" between mass accretion through the shock and mass settling onto the PNS. The shock now slowly expands to $140 \mathrm{~km}$ at $60 \mathrm{~ms}$, see Fig. 13. Then, however, the shock slowly retreats again as a consequence of the high ram pressure. The neutrino heating in a relatively narrow gain layer (see also $\delta_{t} E_{\mathrm{gl}}$ in Fig. 14) is too weak to support shock expansion to larger radii. At $170 \mathrm{~ms}$, when the shock has reached a radius of less than $80 \mathrm{~km}$, we see a transient reexpansion of the shock, which is stopped again after a few $\mathrm{km}$. This feature results from a sudden drop of the density in the progenitor (see Fig. 10) and thus of the mass accretion rate (Fig. 17) and of the ram pressure, and is connected with the "interface" between the silicon shell and the oxygen-rich silicon shell (i.e. the $\mathrm{Si}-\mathrm{SiO}$ interface at $1.42 M_{\odot}$ ). Such a phase of transient shock expansion can be seen in all our models, with strongly differing consequences (even explosions, see Sect. 3.2.2). In Model s15Gio_1d.b the shock finds a new equilibrium radius at only $90 \mathrm{~km}$ to then follow the subsequent contraction of the nascent neutron star (visible from the decreasing neutrinosphere radii, see Fig. 15). 


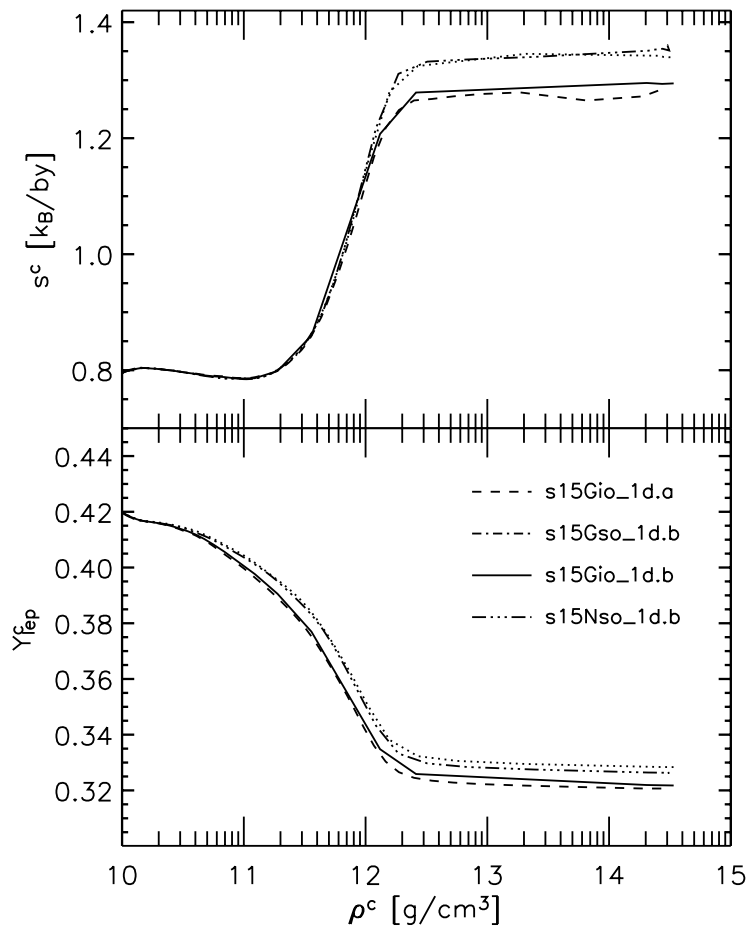

Fig. 12. Evolution of the central values of the entropy (top) and lepton number (bottom) as functions of the central density during the collapse of Models s15Nso_1d.b (dash-triple-dotted), s15Gso_1d.b (dotted), s15Gio_1d.b (solid), and s15Gio_1d.a (dashed).

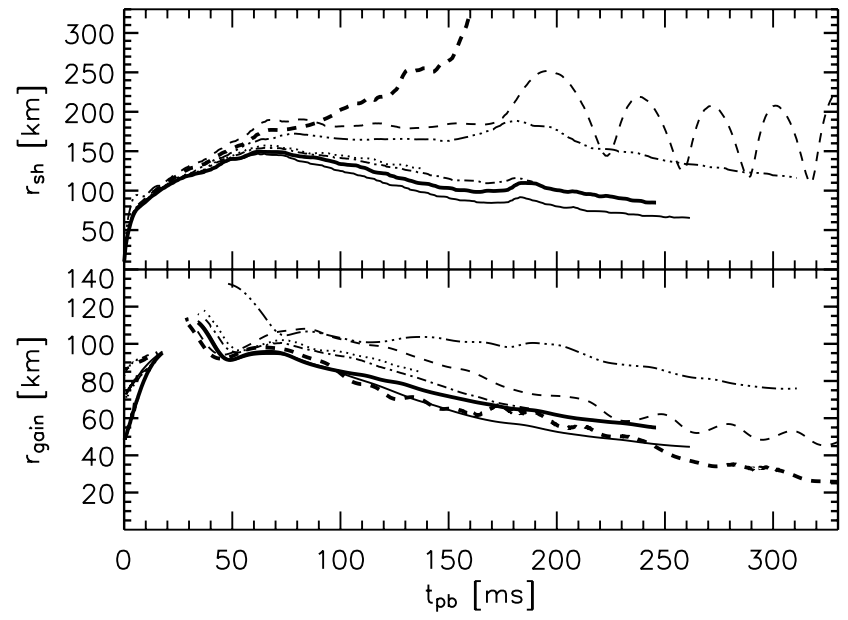

Fig. 13. The top plot shows the shock positions versus time for all presented models. Thin lines are for 1D models, thick lines for 2D, see Fig. 14 for the labelling. The plot below shows the gain radius which separates the neutrino cooling from the neutrino heating layer farther out. Note that the curves in the lower plot were smoothed over intervals of 5ms. The evolution of Models s15Gio_1d.a and s15Gio_32.a was followed to later times. Their complete evolution can be seen in Figs. 28, 36, and 42.

The neutrino emission reflects the different phases of the evolution: after the collapse phase, during which only $v_{\mathrm{e}}$ are emitted in significant numbers, the prompt $v_{\mathrm{e}}$ burst is created few ms after bounce (see Fig. 19; note that the signal is delayed by approximately $1 \mathrm{~ms}$ due to the time-of-flight). The burst lasts approximately $25 \mathrm{~ms}$, has a fwhm of $6 \mathrm{~ms}$, and

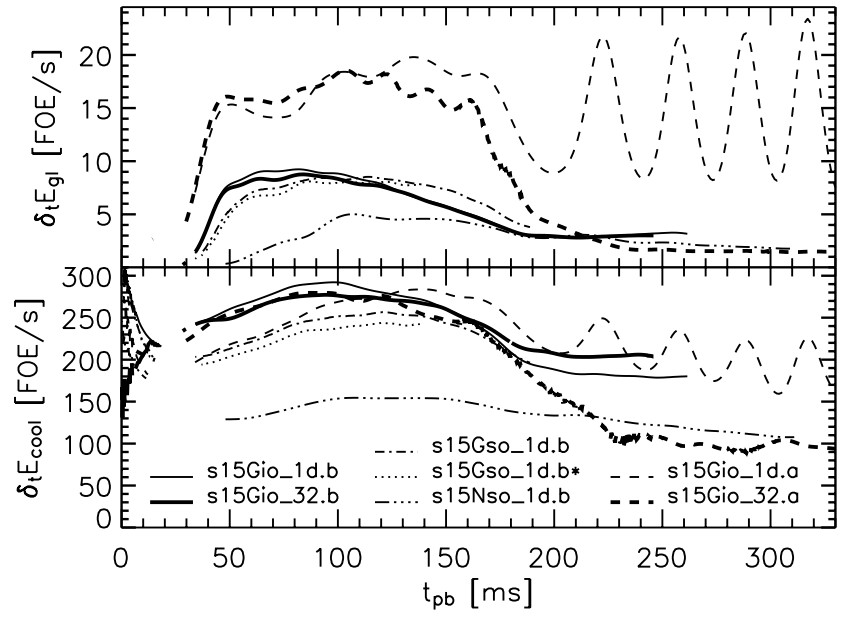

Fig. 14. The top plot shows the total net heating rate in the gain layer, i.e. between the gain radius and the shock. The lower plot shows the total cooling rate below the gain radius. Note that the curves were smoothed over intervals of $5 \mathrm{~ms}$.

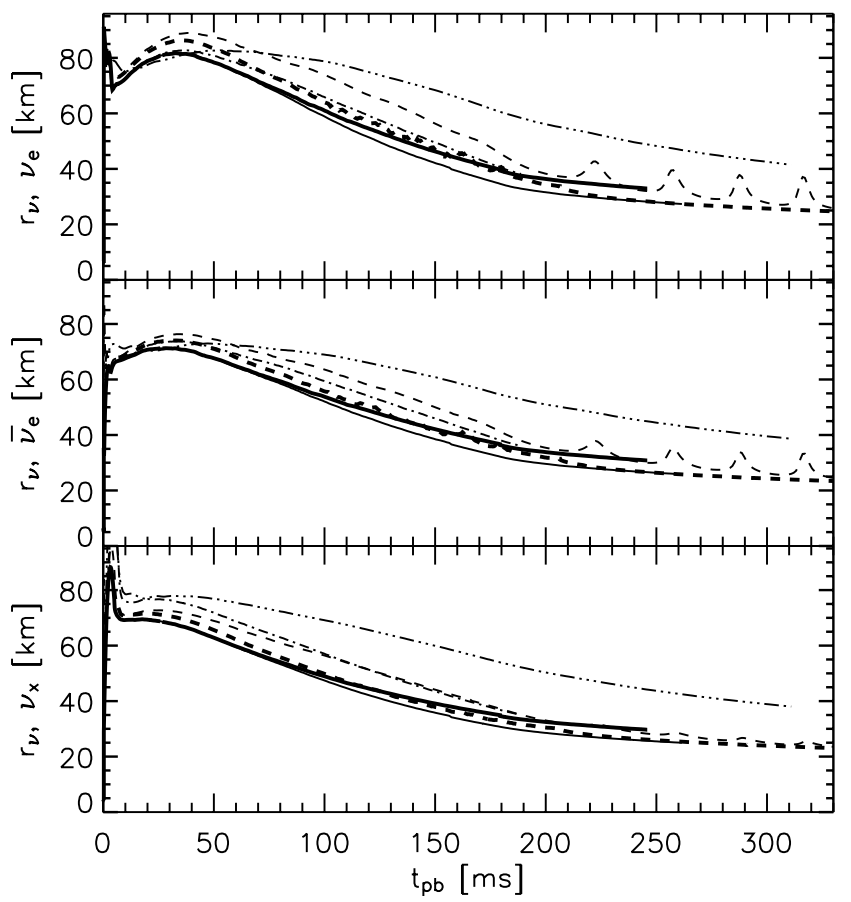

Fig. 15. The three plots show the neutrinosphere radii of all presented models for $v_{\mathrm{e}}$ (top), $\bar{v}_{\mathrm{e}}$ (middle), and $v_{\mu, \tau}$ and $\bar{v}_{\mu, \tau}$ (bottom), see Fig. 14 for the labelling. Note that Model s15Gso_1d.b* is missing in these plots (because of data loss).

reaches a peak luminosity of about $350 \mathrm{FOE} / \mathrm{s}(\mathrm{FOE}=$ ten to the fifty-one ergs), see Fig. 19. Simultaneously, the newly created PNS starts emitting $\bar{v}_{\mathrm{e}}$ and $v_{\mathrm{x}}$. Their rise in luminosity takes $35 \mathrm{~ms}$ and $15 \mathrm{~ms}$, respectively. In the following accretion phase, the emitted neutrinos come from two regions, from a layer below the neutrinosphere "inside" the cooling PNS and to a smaller part from the layer of newly accreted material between the PNS surface and the gain layer. The luminosity produced by the latter source depends sensitively on the mass accretion rate as can be seen in the drop of the luminosity when the $\mathrm{Si}-\mathrm{SiO}$ interface enters the cooling region. Interestingly, the 
mean energies of $\bar{v}_{\mathrm{e}}$ and $v_{\mathrm{x}}$ become very similar after about $100 \mathrm{~ms}$ post-bounce. This fact has been discussed in detail in Keil et al. (2003) and will be addressed in more detail below (Sect. 3.1.2).

Looking more closely at our simulation of Model s15Gio_1d.b, we find that its outcome is not very surprising. After shock stagnation and during the whole subsequent evolution we see that the shock is unable to stop the rapid infall of the accreted material: behind the shock, the matter still has negative velocities of several $1000 \mathrm{~km} \mathrm{~s}^{-1}$, thus falling quickly through the narrow gain layer. As an example, at the time of maximal shock expansion at $72.5 \mathrm{~ms}$, the infall velocity behind the shock is still $4 \times 10^{3} \mathrm{~km} \mathrm{~s}^{-1}$ (see Fig. 20), and the short distance between shock and gain radius of at most $50 \mathrm{~km}$ corresponds to an advection timescale through the gain layer of less than $15 \mathrm{~ms}$. With a moderate neutrino heating rate of $300 \mathrm{MeV} / \mathrm{s}$ per baryon only about $4.5 \mathrm{MeV}$ per baryon can be deposited in these $15 \mathrm{~ms}$, an amount which is clearly insufficient to promote shock expansion: at the high entropies $\left(>10 k_{\mathrm{B}} /\right.$ by) behind the shock nuclei are nearly completely dissociated to nucleons. Only a small fraction of $\alpha$-particles (less than 20\%) survive the shock passage, and are quickly dissociated during infall. Thus, in the shock the infalling matter loses 8-9 MeV per baryon due to nuclear photo-dissociation.

\subsubsection{Variations of the input physics}

"Standard" opacities vs. improved opacities. We have performed most of our calculations either using a set of neutrino interactions which we call "standard opacities" (Case so) or our fully updated ("state-of-the-art") description of neutrino interactions which we call "improved opacities" (Case io) ${ }^{1}$. The treatment of neutrino interactions in Case io is described in Appendix A. Case so uses the neutrino interactions from Bruenn (1985) and Mezzacappa \& Bruenn (1993a,b), implemented as described by Rampp \& Janka (2002), but supplemented by neutrino pair creation and annihilation due to nucleon-nucleon bremsstrahlung. In neither of our simulations we take into account neutrino pair creation by the plasmon process because this process is negligible for $v_{\mathrm{e}}$ and $\bar{v}_{\mathrm{e}}$, and for $v_{\mathrm{x}}$ it is either dominated by nucleon-nucleon bremsstrahlung (at high densities) or by the flavor coupling $v \bar{v}$ pair process $\left(v_{\mathrm{x}} \bar{v}_{\mathrm{x}} \leftrightarrow v_{\mathrm{e}} \bar{v}_{\mathrm{e}}\right.$; at low densities). For a recent calculation of the plasmon process at SN-relevant conditions see the paper by Ratković et al. (2003).

In order to compare the properties of the different interaction rates we have plotted in Figs. 21 and 22 the opacities (inverse mean free paths) of all reactions included in Model s15Gio_1d.b for $v_{\mathrm{e}}, \bar{v}_{\mathrm{e}}$, and heavy-lepton neutrinos $\left(v_{\mathrm{x}}\right)$ for two different energies and for the conditions present in the model at two different post-bounce times. For completeness, we also show the rates for neutrino scattering and absorption on nucleons as used in Case so, i.e. the rates with

1 The new treatment of electron captures on nuclei during core collapse with rates from Langanke et al. (2003), however, is not yet included in any of our models presented here. the approximations of ignoring nucleon recoil and thermal motions, weak magnetism, effective nucleon masses, and nucleonnucleon correlations. During the discussion, remember that absorption and emission are linked by detailed balance. Phase space blocking of neutrinos (and other leptons) in the final channels of the reactions is included in all reactions.

Charged-current absorption processes with nucleons are the dominant mode of destroying $v_{\mathrm{e}}$ and $\bar{v}_{\mathrm{e}}$ after bounce, when the post-shock material is essentially fully dissociated. However, we can see that in the PNS core, the improved rates are reduced significantly compared to the "standard" rates of Case so, for $v_{\mathrm{e}}$ by a factor of up to 30 , for $\bar{v}_{\mathrm{e}}$ even by up to 100 . In the region around the neutrinosphere, including the gain layer, recoil and weak magnetism reduce the $\bar{v}_{\mathrm{e}}$ absorption rate by $10-20 \%$, while the effects nearly compensate each other in case of $v_{\mathrm{e}}$ (compare also Appendix A and Horowitz \& Li 1999). Absorption on nuclei is dwarfed by nucleon absorption even in the pre-shock material. However, our rates for $v_{\mathrm{e}}$ absorption by nuclei are still rather approximative (not taking into account recent advances of electron capture rate calculations by Langanke et al. 2001) and $\bar{v}_{\mathrm{e}}$ capture by nuclei is neglected. Improving this treatment is desirable.

Neutrino-antineutrino pairs can be produced and destroyed in different charged- and neutral-current reactions. One of these is nucleon-nucleon bremsstrahlung, which contributes significantly to the emitted flux of heavy-lepton neutrinos $v_{\mathrm{x}}$ (Thompson et al. 2000; Keil et al. 2003; Thompson et al. 2003) because this process dominates the production of $v_{\mathrm{x}}$ at high densities. Interestingly, bremsstrahlung is also the dominant absorption reaction for $\bar{v}_{\mathrm{e}}$ in the PNS core, where the electron degeneracy is very high and therefore $v_{\mathrm{e}}$ are orders of magnitudes more abundant than $\bar{v}_{\mathrm{e}}$. It is also the dominant production rate since positrons are very rare in the degenerate region, leading to a strongly suppressed rate of $\mathrm{e}^{+}+\mathrm{n} \rightarrow \bar{v}_{\mathrm{e}}+\mathrm{p}$. The second pair process is the annihilation of $v \bar{v}$ pairs to $\mathrm{e}^{+} \mathrm{e}^{-}$pairs. The inverse reaction dominates bremsstrahlung in creating $\bar{v}_{\mathrm{e}}$ at low densities and is also a very important process for the generation of $v_{\mathrm{x}}$. This rate, however, is surpassed by the rate of $v \bar{v}$ pair conversion between different flavors, which in most regimes is approximately a factor of two more important for producing $v_{\mathrm{x}} \bar{v}_{\mathrm{x}}$ pairs than $\mathrm{e}^{+} \mathrm{e}^{-}$annihilation. Both rates dominate the $v_{\mathrm{x}}$ production around the neutrinosphere, thus being crucial in the spectrum formation process of these neutrinos (cf. Keil et al. 2003; Buras et al. 2003a). For higher energies, the leptonic pair production rates become increasingly important relative to bremsstrahlung.

Finally, scattering processes contribute to the neutrino opacity. Scattering of neutrinos on nuclei dominates the scattering rates above the shock. Scattering on nucleons are the dominant rates in the NS core and postshock layer. Dense medium effects (see Appendix A), however, reduce the rate by a factor of 2-3 at high densities below the neutrinosphere and phase space blocking effects lead to a dramatic reduction for scattering rates for $v_{\mathrm{e}}$ in case of nucleon recoil being included. Again, weak magnetism and recoil produce a $10 \%$ difference for $\bar{v}_{\mathrm{e}}$-nucleon scattering around the neutrinosphere, and a much smaller effect for $v_{\mathrm{e}}$ (see Appendix A). Scattering 


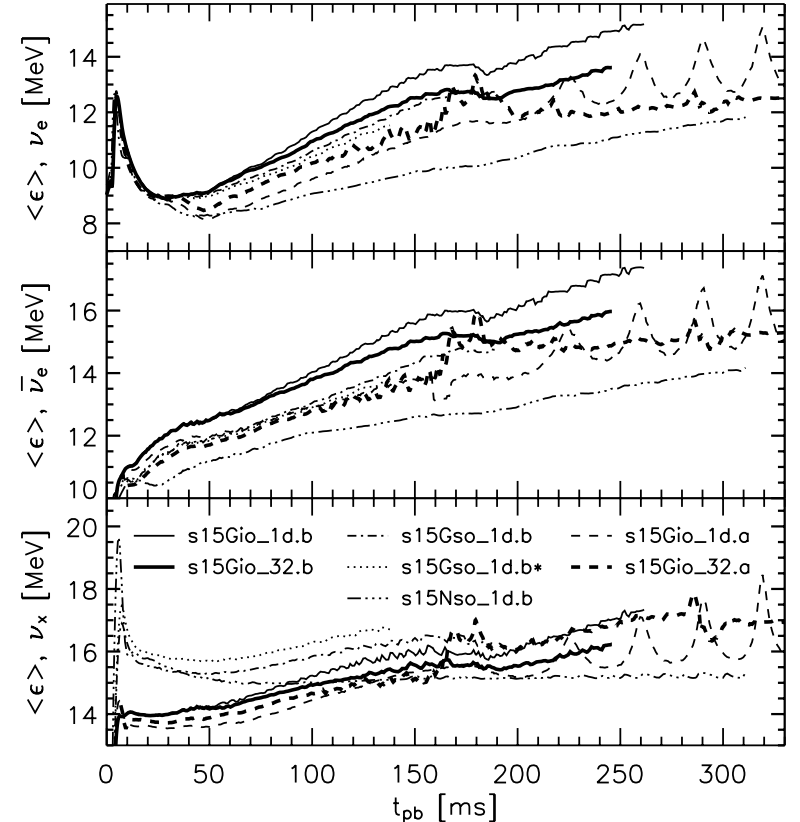

Fig. 16. The three plots show the average energies of the emitted $v_{\mathrm{e}}$ (top), $\bar{v}_{\mathrm{e}}$ (middle), and $v_{\mu, \tau}$ or $\bar{v}_{\mu, \tau}$ (bottom) as measured by an observer at rest at $400 \mathrm{~km}$.

off electrons and positrons and off $v_{\mathrm{e}}, \bar{v}_{\mathrm{e}}$ are of similar importance for $v_{\mathrm{x}}$ (see also Buras et al. 2003a) ${ }^{2}$.

At higher neutrino energies the different opacities show similar behaviour (Fig. 21). The suppression of neutrino absorption on nucleons at high densities is weaker (because phase space blocking is less strong), and bremsstrahlung processes are less important compared to other processes. At later times the temperature in the postshock layer increases and $\mathrm{e}^{-}$degeneracy is reduced so that the $\mathrm{e}^{+} \mathrm{e}^{-}$pair process and $v \mathrm{e}^{ \pm}$scattering become more important (compare Figs. 22 with 21).

The influence of the neutrino interactions can be seen in dynamical simulations with different input physics (Figs. 13-16, 18, 19). In addition to the above presented Model s15Gio_1d.b with our set of "improved" opacities, we have performed two dynamical simulations s15Gso_1d.b and s15Gso_1d.b*, which both were run with the traditional approximations for the opacities. In the latter case bremsstrahlung was also neglected in order to use the rate input of previous simulations by other groups. The influence of nucleon-nucleon bremsstrahlung was already discussed by Keil et al. (2003) and Thompson et al. (2003), the importance of interactions between neutrinos of different flavors was pointed out by Buras et al. (2003a).

The collapse phase is hardly influenced by the use of our improved description of neutrino-nucleon interactions, because free nucleons are not very abundant during core

${ }^{2}$ Note that the strong decrease of the $v_{\mathrm{x}} v \rightarrow v_{\mathrm{x}} v$ rate at large radii $(\gtrsim 80 \mathrm{~km}$ ) where the process is essentially unimportant is due to an artificial suppression of this rate with a factor of $\left(1+\rho_{10}^{-3}\right)^{-1}$ where $\rho_{10}=\rho /\left(10^{10} \mathrm{~g} \mathrm{~cm}^{-3}\right)$. This procedure was necessary because our rate implementation works correctly only when electron neutrinos are in chemical equilibrium.

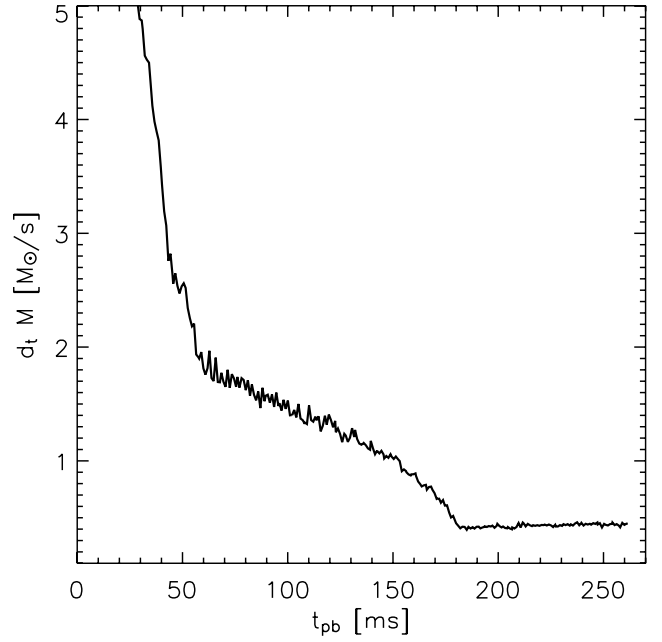

Fig. 17. Mass accretion rate through the shock for Model s15Gio_1d.b. For the other models its evolution is nearly the same, unless an explosion occurs.

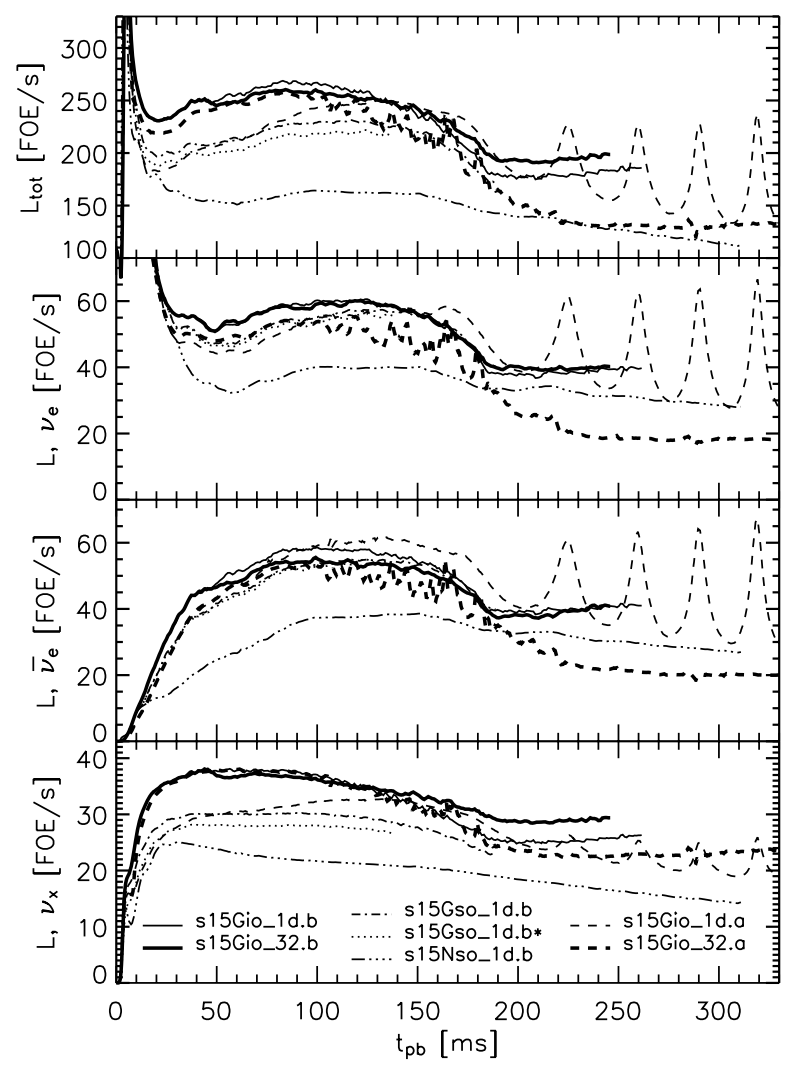

Fig. 18. Comparison of the neutrino luminosities for all models for an observer at rest at $400 \mathrm{~km}$. The plot on top shows the total luminosities, the three lower ones those for the different $v$ types. The plot at the bottom gives the luminosities of $v_{\mu}, \bar{v}_{\mu}, v_{\tau}$, or $\bar{v}_{\tau}$, which are all assumed to be equal.

collapse $\left(Y_{\mathrm{n}}+Y_{\mathrm{p}} \simeq 10^{-3}\right)^{3}$. The improvement of the electron capture rate on protons slightly increases the $v_{\mathrm{e}}$ production below the trapping density (see Fig. A.4). With the coherent scattering rate, which dominates the opacity during infall, not being

\footnotetext{
${ }^{3}$ During core collapse until bounce, our simulations were run without $\bar{v}_{\mathrm{e}}$ and $v_{\mathrm{x}}$, both being irrelevant during this phase.
} 

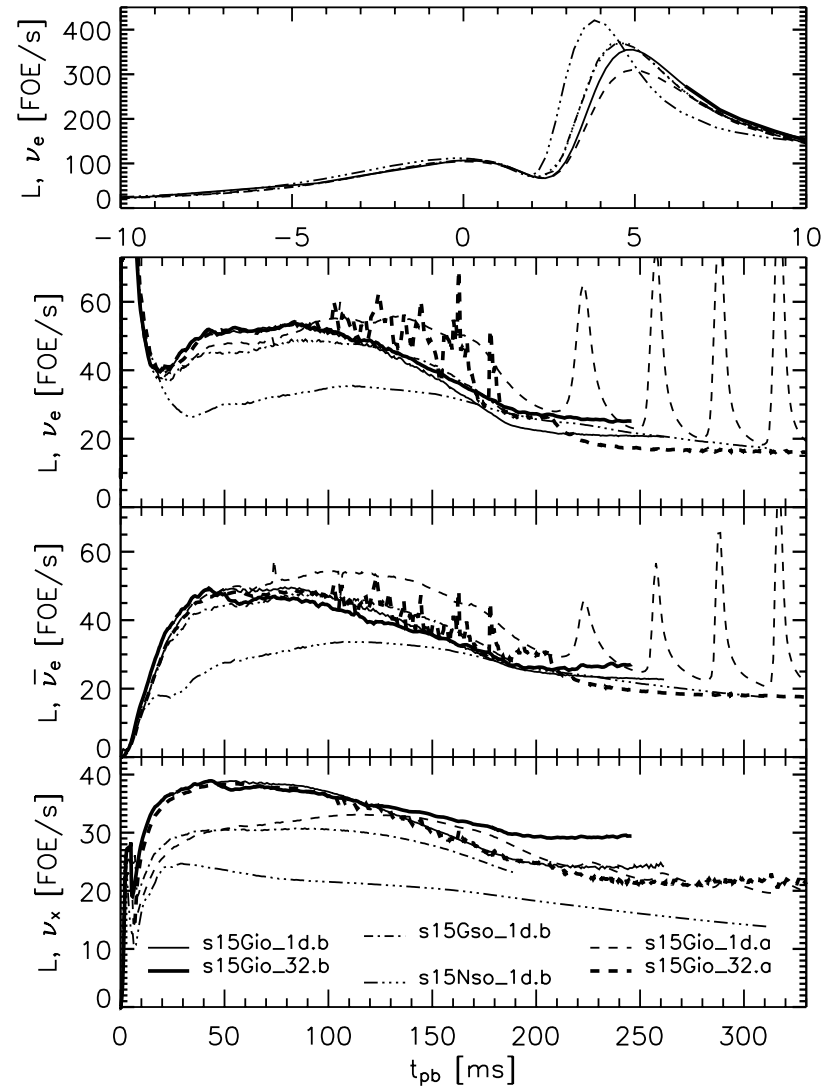

Fig. 19. The uppermost plot shows the electron neutrino burst, again for an observer at rest at $400 \mathrm{~km}$. Note the different scale on the abscissa of this plot. The lower three plots display the neutrino luminosities of $v_{\mathrm{e}}, \bar{v}_{\mathrm{e}}$, and heavy-lepton neutrinos individually, evaluated at their respective neutrinospheres (for a comoving observer). Note that the differences compared to the luminosities shown in Fig. 18 come mostly from neutrino emission and absorption in the cooling and heating layers outside of the neutrinosphere (and not from observer frame motion). Also note that Model s15Gso_1d.b* is missing in the lower three plots (due to missing data).

changed, more $v_{\mathrm{e}}$ are created and escape before the matter becomes optically thick. As a consequence, the simulation with improved opacities yields slightly lower entropies $(|\Delta s| \simeq 0.06)$ and a slightly decreased lepton fraction $\left(\left|\Delta Y_{\text {lep }}\right| \lesssim 0.01\right)$ in the core after trapping compared to the simulation with standard opacities (Fig. 12). Therefore the collapse time also decreases $\left(\left|\Delta t_{\text {coll }}\right| \simeq 2.3 \mathrm{~ms}\right)$ and the homologous core becomes slightly smaller, which reduces the mass enclosed by the shock formation radius $\left(\left|\Delta M_{\mathrm{sc}}\right| \simeq 0.02\right)$.

After bounce only the first $50 \mathrm{~ms}$ are suited for comparing the models, in this early phase the structure of the PNS (and the above lying layer where the neutrinospheres are located) is still very similar in all general relativistic models.

The lowest panel in Fig. 16 shows that the models cluster into two categories concerning the mean $v_{\mathrm{x}}$ energies. The decrease by $\sim 2 \mathrm{MeV}$ can be explained mainly by the effects of the neutrino-nucleon interactions (without bremsstrahlung) when energy transfer by nucleon recoil in $v-\mathrm{n}$, p scattering is taken into account (see also Raffelt 2001). Bremsstrahlung (compare models s15Gso_1d.b* and s15Gso_1d.b) and the flavor-coupling neutrino reactions (compare models ${ }^{4}$ s15Gio_1d.a and s15Gio_1d.b) have a small effect on $\left\langle\epsilon_{v_{x}}\right\rangle(\sim 0.5 \mathrm{MeV})$. The mean electron type neutrino energies are hardly changed by the improvements of the neutrino opacities, only the mean energy of $\bar{v}_{\mathrm{e}}$ increases slightly by $\sim 0.7 \mathrm{MeV}$.

Similarly, the $v_{\mathrm{e}}$ spectrum is not altered by the improvements of the opacities, see Fig. 23. For $\bar{v}_{\mathrm{e}}$ the high-energy flux increases by a factor of three, being consistent with the increase of $\left\langle\epsilon_{\bar{v}_{\mathrm{e}}}\right\rangle$. The most significant change can again be seen for $v_{\mathrm{x}}$. We observe a pinching of the spectrum (higher peak, steeper drop at high energies).

Concerning the luminosities, see Fig. 18, we again find a clustering of the models in case of the $v_{\mathrm{x}}$ luminosity. Here, however, the increase by $25-30 \%$ comes from the interactions between the neutrinos of different flavors, whereas the improved neutrino-nucleon interactions have a minor $(<10 \%)$ influence (compare s15Gso_1d.b* with s15Gio_1d.a). Again, the effects on $v_{\mathrm{e}}$ and $\bar{v}_{\mathrm{e}}$ are much smaller. For both $v_{\mathrm{e}}$ and $\bar{v}_{\mathrm{e}}$ the luminosities increase by less than $10 \%$.

As a direct consequence of the increased luminosities, the cooling rate of the PNS, $\partial_{t} E_{\text {cool }}$ in Fig. 14, is $25 \%$ higher in case the interactions between neutrinos of different flavors are turned on. The effect on the evolution of the shock, however, is very weak and becomes visible only later than $100 \mathrm{~ms}$ after bounce when the enhanced contraction of the PNS in models including the flavor-coupling neutrino interactions has altered the structure of the star between neutrinosphere and shock.

Newtonian vs. general relativistic simulations. Finally we discuss the differences arising between simulations with our approximative treatment of general relativity (GR) and Newtonian calculations by comparing Models s15Gso_1d.b with s15Nso_1d.b. In the Newtonian simulation, the gravitational potential is less deep and the onset of core collapse is not supported by relativistic destabilization. Hence the collapse time (until shock formation) of $196.1 \mathrm{~ms}$ is longer compared to $177.7 \mathrm{~ms}$ for the relativistic potential. Nevertheless the central lepton fraction after trapping is nearly the same (it is smaller by only $\Delta Y_{\text {lep }} \simeq 0.002$ in the Newtonian case, see Fig. 12), suggesting a very similar enclosed mass at shock formation. However, due to the lower gravitational forces and infall velocities the shock forms at an enclosed mass of $M_{\mathrm{sc}}=$ $0.62 M_{\odot}$ and a radius of $r_{\mathrm{sc}}=12.5 \mathrm{~km}$ in the Newtonian case, whereas it is launched at $M_{\mathrm{sc}}=0.51 M_{\odot}$ and $r_{\mathrm{sc}}=10.8 \mathrm{~km}$ in the GR simulation.

The less compact structure of the PNS in the run with the Newtonian gravitational potential strongly influences the postbounce evolution: the neutrinospheres and the accretion shock stand at larger radii, the increase of temperature and entropy in the gain layer is slower, and the neutrino luminosities are lower, see Figs. 13, 15, 18, and 24. These results agree with the more elaborate discussion of differences between Newtonian and GR simulations by Liebendörfer et al. (2001).

\footnotetext{
${ }^{4}$ Note that the omission of $\beta$-dependent terms has no noticeable influence on the layers of neutrino emission at early post-bounce times.
} 

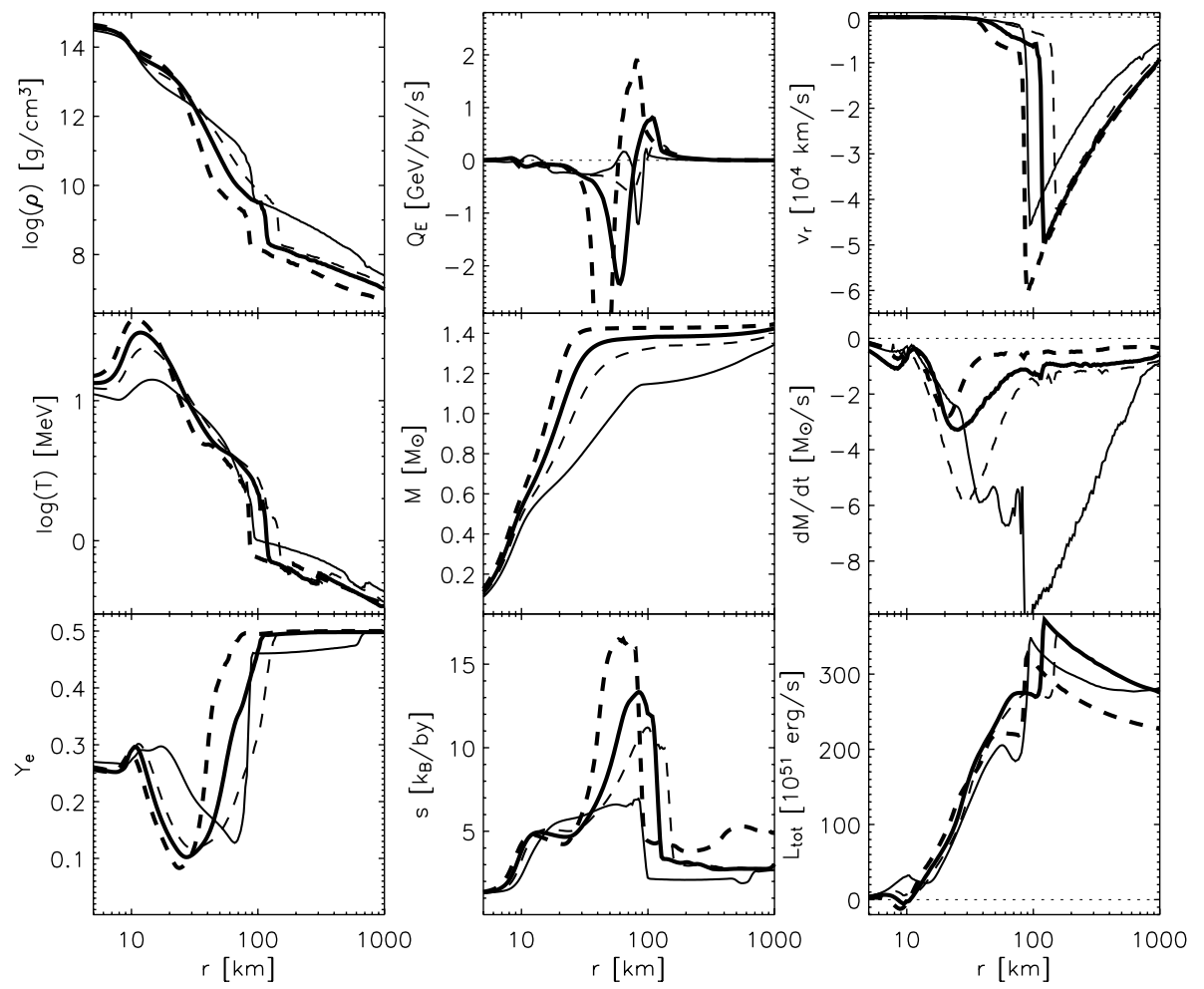

Fig. 20. Stellar profiles of different quantities at post-bounce times of $11.6 \mathrm{~ms}$ (solid), $72.5 \mathrm{~ms}$ (dashed), $113.7 \mathrm{~ms}$ (thick solid), and $170.7 \mathrm{~ms}$ (thick dashed) versus radius for our reference Model s15Gio_1d.b. For the latest time the cooling rate reaches a maximum value of $Q_{\mathrm{E}}=$ $-10 \mathrm{GeV} / \mathrm{s}$ per baryon.

\subsubsection{Velocity-dependent terms in the first order moments equation}

Terms which contain $\beta$ in the first order moments equation of the transport equation (except for the $\beta \partial / \partial r$-terms) are usually considered to be small and therefore negligible for many problems when $v / c \ll 1$ (Mihalas \& Mihalas 1984; see also the discussion in Rampp \& Janka 2002). However, in the presented supernova simulations, we found that the velocities are sufficiently large in the neutrino decoupling and heating layers, which are crucial for the explosion, that these " $\beta$-terms" can make a big effect. With " $\beta$-terms" we mean here all terms depending on $\beta_{r}$, except the advection terms, which are proportional to $\beta_{r} \partial / \partial r$ and which are included in all calculations. The omission of these $\beta$-terms in the neutrino momentum equation turned out to change the dynamical evolution of the supernova and to lead to an artificial explosion in 1D and 2D (Sects. 3.1.4 and 3.2.2). Having in mind that in the $O(v / c)$ approach all velocity-dependent first-order terms in the radiation energy equation are known to be relevant (see Mihalas \& Mihalas 1984 ) and the importance of the $\beta_{\vartheta}$-terms was recognized in Sect. 2.3.2, we therefore conclude that all terms of first order in the fluid velocity must be taken into account in supernova simulations ${ }^{5}$. Doing so, we found that our transport code produces results in very good agreement with spherically symmetric simulations with the Oak Ridge/Basel AGILE-BOLTZTRAN code

${ }^{5}$ We point out that some authors think that going even beyond the $O(v / c)$ approach of our work is desirable (cf. Cardall et al. 2005). for Newtonian as well as relativistic gravity (Liebendörfer et al. 2005; Marek et al. 2006).

We want to investigate the role of the $\beta$-terms in the neutrino momentum equation, Eq. (B.14), more closely with a snapshot of Model s15Gio_1d.b at $t_{\mathrm{pb}}=114 \mathrm{~ms}$, for which we have evaluated these terms at energies near the spectral maxima of neutrinos and antineutrinos, see Fig. 25. For $v_{\mathrm{e}}$ and $\bar{v}_{\mathrm{e}}$ the $\beta$-terms account for up to $20-30 \%$ of the LHS of Eq. (B.14) in the neutrino-heated region between 70 and $110 \mathrm{~km}$; for muon neutrinos, which do not contribute to the heating at a significant level, the $\beta$-terms can even dominate the LHS of Eq. (B.14). The individual size of these terms at $114 \mathrm{~ms}$ after core bounce is visible in Fig. 25d. One can see that the different terms have different relative importance in different regions of the star. We emphasize that it can therefore not be concluded that any of these terms can be safely ignored for all situations.

The role of the $\beta$-terms can be made more transparent if we write them on the RHS and compare them with the source term for momentum exchange. In regions with negative velocity and negative velocity gradient, the $\beta$-terms have the opposite sign of the source terms in Eq. (B.14) and therefore effectively reduce the coupling between matter and neutrino flux. Thus, neglecting the $\beta$-terms has the opposite effect, leading to a less rapid streaming of neutrinos and an increased energy density of neutrinos above and below the gain radius. Note that for higher energies, the velocity terms become less important.

These statements are confirmed by Fig. 26, where we show stationary transport solutions calculated for the considered stellar profile both with and without the $\beta$-terms. The neutrino 


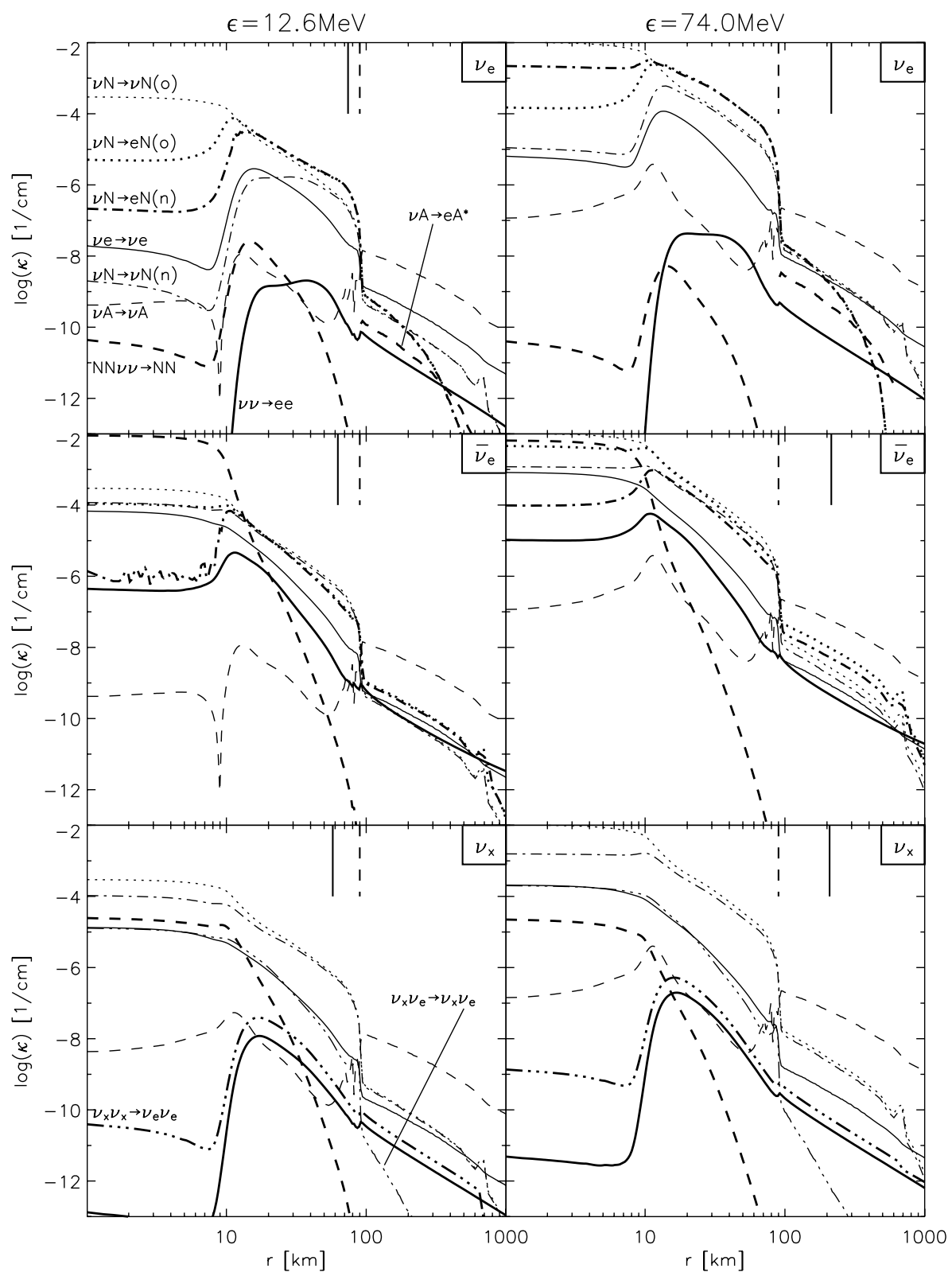

Fig. 21. Opacities for the neutrino interactions taken into account in Model s15Gio_1d.b at $t=11.6$ ms post-bounce for two representative neutrino energies. Neutrino (" $v$ ") reactions with electrons ("e", solid), nucleons ("N", dash-dotted), nuclei ("A", dashed), and electron-type neutrinos (" $v_{\mathrm{e}}$ ", dash-triple-dotted, for $v_{\mathrm{x}}$ only) are shown. For comparison the "standard" opacities for the neutrino-nucleon interactions are also displayed (dotted). Thin lines represent scattering processes, thick lines correspond to absorption processes. Note that different from this convention nucleon-nucleon bremsstrahlung (important only below the shock) is also represented by thick dashed lines. (See also caption of Fig. 22).

flux $H$ and the flux factor $H / J$ are larger in the solution with the $\beta$-terms, and the neutrino density is smaller, which directly decreases the neutrino heating (Fig. 26b). From the plots, it can also be seen that the solution with the $\beta$-terms is consistent with physical requirements: since the neutrino quantities are given in a comoving frame, both luminosity and energy density reveal a larger blue-shifting ahead of the shock where the infall velocities are higher. The corresponding "step" at the shock, however, is absent in case of $J$ in the solution without $\beta$-terms (Fig. 26a, left panel). The flux factor is affected by these differences. Without $\beta$-terms it clearly disagrees with results of transport calculations with an $S_{N}$ solver for the Boltzmann equation (see
Liebendörfer et al. 2005). In contrast, the variable Eddington factor $f_{\text {ed }}=K / J$ turns out not to reveal the same degree of sensitivity to the $\beta$-terms.

The $\beta$-terms were found to have a dramatic dynamical effect. In Fig. 13 we see the evolution of Models s15Gio_1d.a and s15Gio_1d.b, which mostly differ in the fact that only Model " $\mathrm{b}$ " includes the $\beta$-terms ${ }^{6}$. We see that the shock positions evolve increasingly differently. Initially, in Model "a" the post-shock heating by neutrinos is higher, see Fig. 14.

${ }^{6}$ The disregard of flavor-coupling neutrino processes in Model s15Gio_1d.a is not very relevant in the present context. 


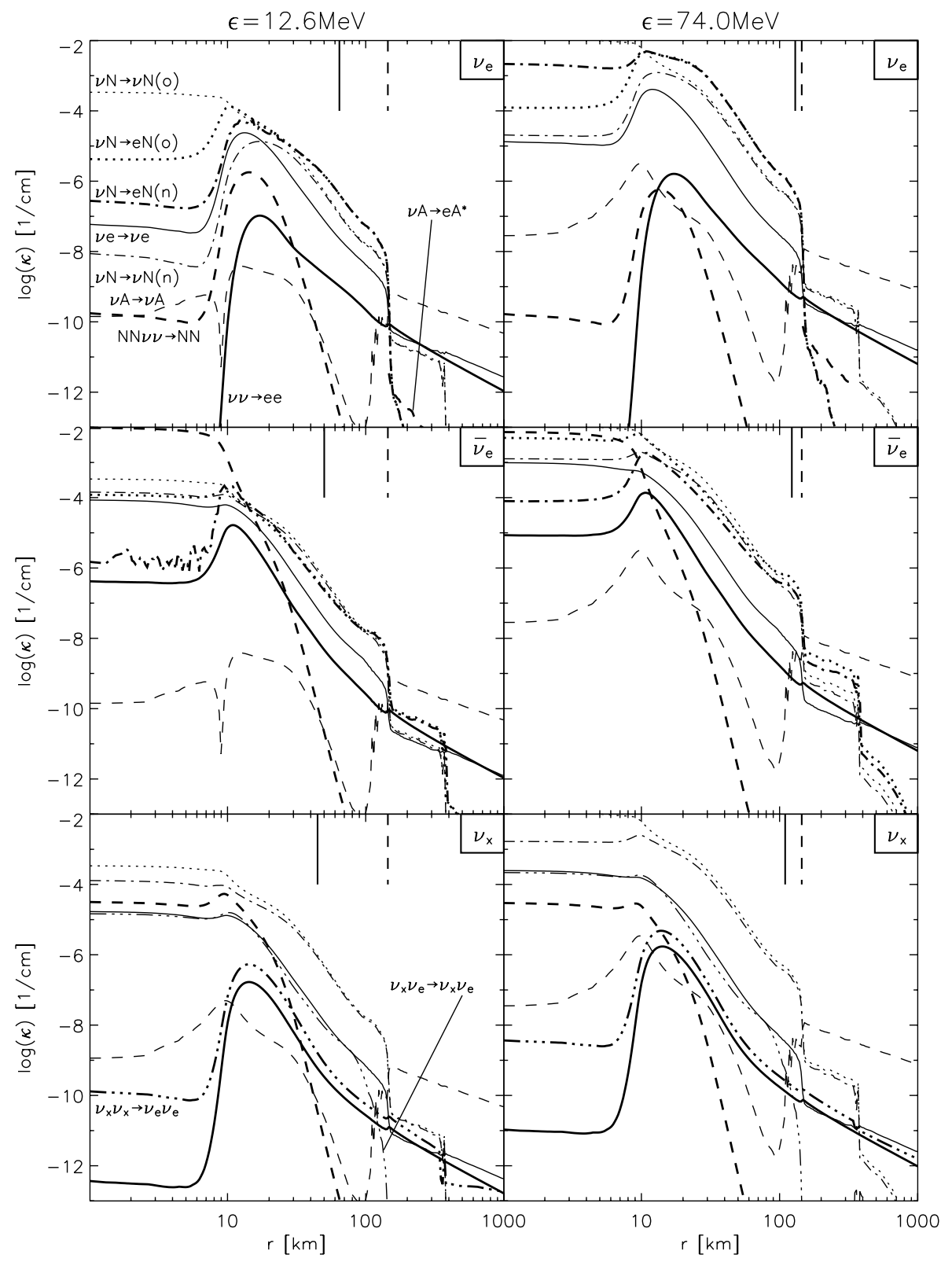

Fig. 22. Same as Fig. 21, but for $t=73.4 \mathrm{~ms}$ after bounce. The opacities include processes with all possible interaction partners: " $\mathrm{N}$ " stands for nucleons and can represent protons and/or neutrons, "e" can be electrons and/or positrons, "A" represents all nuclei, " $v$ " can be any type of neutrino or antineutrino, " $v_{\mathrm{e}}$ " can be electron neutrinos and/or antineutrinos, and " $v_{\mathrm{x}}$ " can be any type of heavy lepton neutrino or antineutrino. The opacities include all phase-space blocking factors. The short vertical lines on the top of the plots indicate the radii of the neutrinosphere for the given neutrino type and energy (solid) and of the shock (dashed).

At later times, the increased heating has driven the shock farther out, thus increasing the size and mass of the gain layer. The resulting positive feedback between increasing gain layer and thus increasing heating on the one hand and expanding shock radius on the other leads to a much larger shock radius than in Model "b", where the contracting shock causes the gain layer to become more and more narrow. During the phase of rapid accretion until about $180 \mathrm{~ms}$ post-bounce, the total heating rate in the gain layer is larger in Model s15Gio_1d.b by a factor of about two, and even larger at later times.

This analysis is confirmed when Models s15Gio_1d.b (with $\beta$-terms) and s15Gio_1d.a are compared at $t=73 \mathrm{~ms}$ postbounce (Fig. 27): the flux factor $f_{H} \equiv H / J$ in the former model increases much faster in the regions of effective neutrino cooling and heating than in Model s15Gio_1d.a. The lower neutrino energy density corresponds to a significantly reduced integral rate of neutrino heating and a much reduced rate of cooling below the gain radius.

\subsubsection{A model with marginal behaviour}

Although Model s15Gio_1d.a was computed with reduced accuracy in the neutrino transport (the $\beta$-terms in the first order moments equation were neglected), its evolution deserves a discussion. It reveals an oscillating shock behaviour with growing amplitude (similar to what was previously seen in models of 


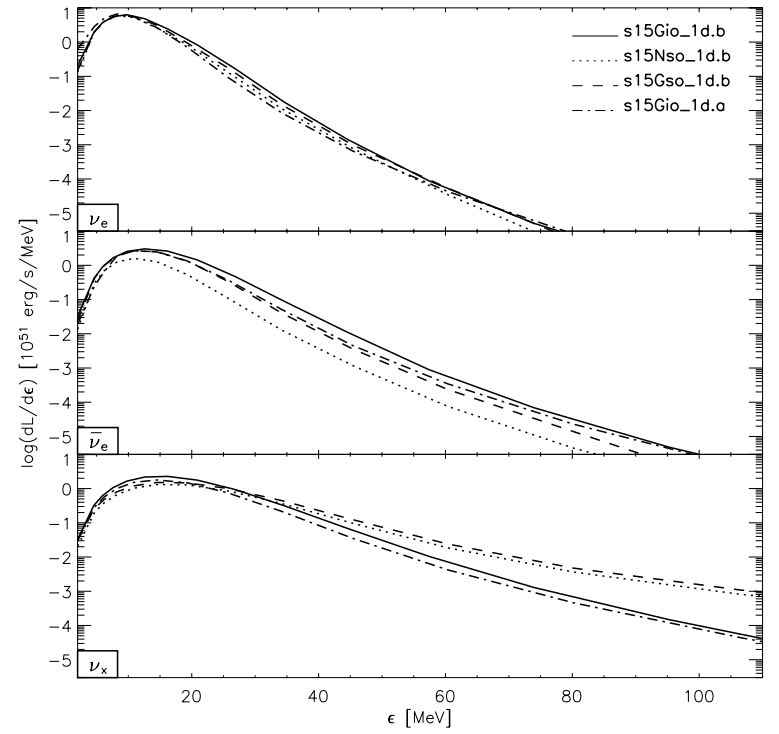

Fig. 23. Neutrino flux spectra for an observer at rest, evaluated at a radius of $400 \mathrm{~km}$ at a time of $30 \mathrm{~ms}$ after bounce. The different panels display results for $v_{\mathrm{e}}, \bar{v}_{\mathrm{e}}$, and $v_{\mathrm{x}}$, respectively. The different lines correspond to different $1 \mathrm{D}$ models as labeled in the plot.

the Livermore group, see e.g. Wilson et al. 1986), which finally leads to an explosion.

As discussed in Sect. 3.1.3, the omission of the $\beta$-terms causes increased neutrino heating behind the stalled SN shock. The shock is therefore driven farther out before it stagnates at about $180 \mathrm{~km}$. When the interface between silicon layer and oxygen-rich silicon shell arrives at the shock, a sudden decrease of the ram pressure occurs and instigates a sequence of periodic phases of shock expansion and contraction (Figs. 28, 29). Whenever the shock expands and the mass infall is decelerated, the neutrino luminosity drops with a slight delay as a consequence of the decreased mass accretion rate towards the cooling region. In response to the decreased luminosity the heating almost simultaneously drops, partly also because the accreted material expands behind the shock and stays away from the gain radius where heating is most efficient. With the energy input being quenched the matter behind the shock quickly falls inward. Being drained of its pressure support the shock expansion comes to a halt again and the shock retreats. The sudden increase of the mass flow into the cooling region raises the accretion luminosity extremely, and the heating rate reaches up to $20 \%$ of the cooling rate, also enhanced by more mass moving into the region just above the gain radius. The shock bounces and is driven out in a new phase of expansion. This cycle is repeated several times. Obviously, the feedback of the cycle is positive, since the amplitude of the shock expansion increases with time and the cycle finally leads to an explosion.

It is beyond the scope of this work to determine exactly the criterion when such a feedback cycle is obtained and why Model s15Gio_1d.a but no other of our models shows such a vertiginous fate. The fact that the $\beta$-terms are neglected certainly increases the heating, and a sufficiently large driving force seems crucial for entering the oscillatory mode.
We see two possibilities for explaining the phenomenon, however make no effort to perform a detailed analysis of any of these. On the one hand the physical conditions which characterize the expansion and contraction phases resemble the conditions that allow for non-adiabatic vibrational instability $(\kappa$-mechanism) in stellar atmospheres where the outward-going heat flow is modulated by the rhythm of the pulsation and instability occurs if during adiabatic compression the absorption coefficient increases (see, e.g. Kippenhahn \& Weigert 1990, Chap. 39.1).

On the other hand, the model might reveal the action of the so-called acoustic-advective cycle proposed by Foglizzo \& Tagger (2000) for adiabatic accretion flows to black holes. In this scenario, acoustic waves created at the PNS surface propagate to the shock and cause entropy fluctuations there. When these fluctuations are advected to the PNS surface by the accretion flow, they create new acoustic waves and so on. If the feedback is positive, an $l=0$ mode can be built up, as seen in our model. A preliminary analysis by Foglizzo (2003, personal communication) revealed that our oscillating model has favorable conditions for developing an $l=0$ instability by the advective acoustic cycle. However, in our model neutrino heating and cooling dominate and the accretion flow is not at all adiabatic. A more reliable analysis has to account for this fact and remains to be done.

\subsection{Two-dimensional models}

An inspection of the stability criterion (see Sect. 2.3.2 for our specific definition of the stability criterion) for the 1D models (Fig. 30) tells us that the gain layer as well as an extended region in the PNS are convectively unstable, so that multi-dimensional simulations become mandatory. Here, we present the first two-dimensional simulations of convection in supernova cores using a multi-frequency treatment of neutrino transport. The two Models s15Gio_32.a and s15Gio_32.b correspond to the one-dimensional Models s15Gio_1d.a and s15Gio_1d.b, respectively, presented in Sects. 3.1.1 and 3.1.4. Both 2D simulations include our most elaborate description of neutrino-matter interactions (Case "io") as described in Appendix A of this paper and an approximative, spherically symmetric treatment of general relativity (Sect. 2.1). Remember that Model "b" is the one with the full treatment of neutrino transport, while Model "a" incorrectly neglects the $\beta$-terms in radial direction (see Sect. 3.1.3). In the angular direction both models employ 32 zones with a resolution of $2.7^{\circ}$, covering a wedge around the equator from $-43.2^{\circ}$ to $+43.2^{\circ}$. Both models were followed through core collapse in one dimension and mapped to the 2D grid around $7 \mathrm{~ms}$ after the bounce. Simultaneously, perturbations were seeded by randomly changing the radial velocity by up to $\pm 1 \%$ in the entire star. The treatment of the lateral neutrino transport was carried out as described in Sect. 2.2. Note that in Model s15Gio_32.b the inner $2 \mathrm{~km}$ were treated spherically symmetrically to maintain a reasonably large CFL time step. Model s15Gio_32.a did not include the lateral momentum transfer to the fluid, Eq. (18), for which reason the core inside a radius of $25 \mathrm{~km}$ had to 

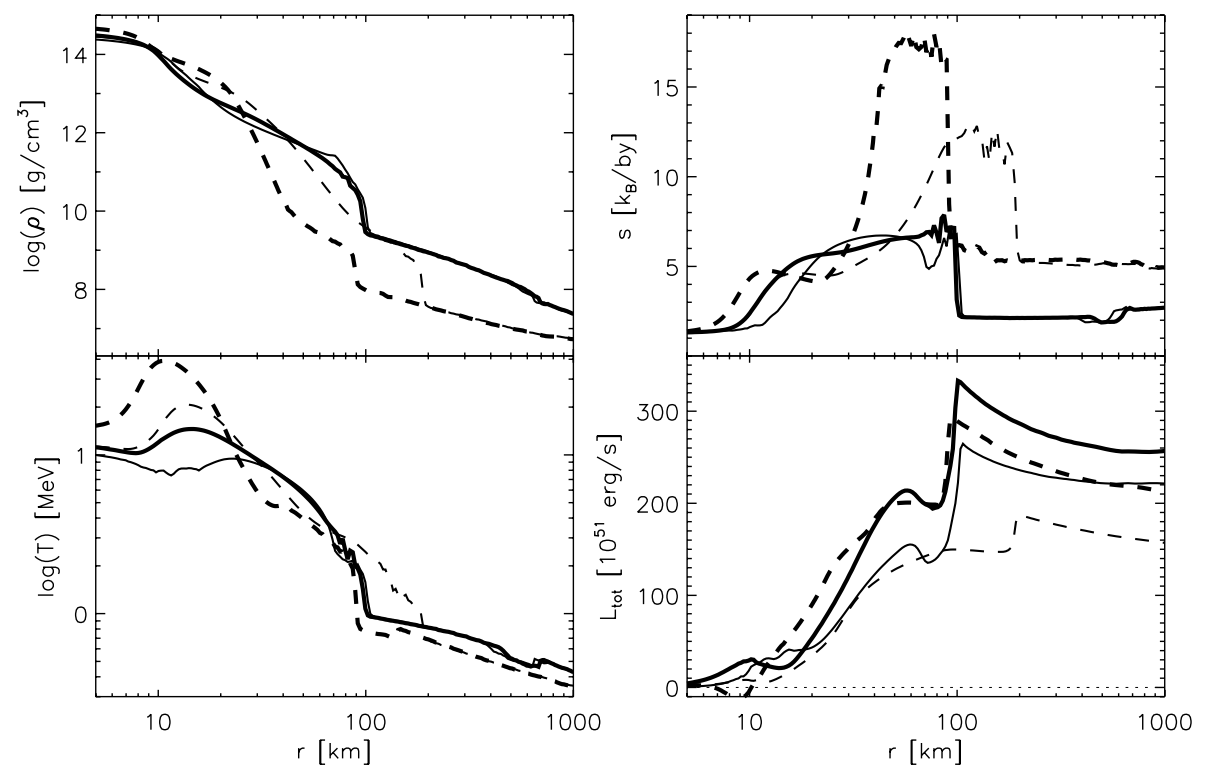

Fig. 24. Comparison of radial profiles for different variables at post-bounce times of $12.3 \mathrm{~ms}$ (solid) and $177.7 \mathrm{~ms}$ (dashed) for Models s15Nso_1d.b (thin) and s15Gso_1d.b (thick). $L_{\text {tot }}$ is the total neutrino luminosity, i.e. the sum of contributions from neutrinos and antineutrinos of all flavors.

be calculated in spherical symmetry. Thus artificial hydrodynamic instability could be avoided, but also the physical PNS convection would not be followed in a layer below the neutrinospheres. Note that the entropy wiggles in the postshock layer discussed in detail in Sect. 2.5 were present in both 2D simulations presented here and introduce artificial perturbations of the stellar models in radial directions. More recent calculations with our improved scheme of treating rest-mass effects in the EoS (Sect. 2.5 and Appendix C), however, showed that these numerical perturbations on the entropy profile did not have an appreciable influence on the development and growth rates of convection in the hot bubble region.

\subsection{1. s15Gio_32.b: a model with full transport treatment}

Model s15Gio_32.b represents a 2D supernova computation with the presently most complete and detailed implementation of spectral neutrino transport in such models. It fails to explode. In Fig. 31 we show the evolution of shock trajectory and "mass shells" of this model. Note that a "mass shell" trajectory of a 2D simulation does not represent the evolution of Lagrangian mass elements as in spherical symmetry but describes the time evolution of the radius of the spherical shell enclosing a certain value of the mass. After the prompt expansion, the shock is pushed slowly outward by accumulating mass until it reaches a maximum radius of $150 \mathrm{~km}$ around $60-70 \mathrm{~ms}$, and then starts to slowly retreat again. This is quite similar to the $1 \mathrm{D}$ case (cf. Fig. 13). Convection in the neutrino-heated layer and in the PNS shifts the shock position farther out by just several $10 \mathrm{~km}$.

The initial hope in performing 2D calculations was that the "hot bubble" (HB) convection in the gain layer would strengthen the shock sufficiently to lead to an explosion, similar to what was found previously by Herant et al. (1994) and Burrows et al. (1995) with neutrino transport being described by grey, flux-limited diffusion. Hot bubble convection was found to be helpful for shock revival by means of transporting energy to the shock, thus enhancing the postshock pressure and increasing the efficiency of neutrino energy deposition: strongly heated material close to the neutrinosphere is carried to the shock in bubbles rising due to buoyancy forces. At the same time narrow downflows passing besides the bubbles feed the region of strong neutrino heating with cool material. 2D calculations also get rid of another big disadvantage of 1D calculations: when the shock starts expanding in 1D calculations, the accretion of fresh material into the cooling region is quenched, the sudden drop in luminosity and thus heating reduces further support for the expanding shock and thus can be fatal for an explosion.

In the current model, although the whole gain layer is convectively unstable from $t_{\mathrm{pb}}=40 \mathrm{~ms}$ on (see Fig. 32a), the convection has difficulties developing: becoming distinct at $60 \mathrm{~ms}$ after bounce, the HB convection (Fig. 33) has not yet grown strong and formed large-scale structures when the shock begins retreating again. Therefore HB convection is unable to push the shock significantly farther out relative to the shock in the 1D simulation. Also during the later evolution the HB convection cannot develop strength because within only a few milliseconds the matter accreted by the shock is advected into the cooling layer and buoyancy forces are not strong enough to enforce significant rise against the rapid infall of the postshock flow. The shock itself shows almost no response to the convection and remains spherically symmetric throughout the run (Fig. 33). The HB convection in this model leads to only small quantitative differences compared to the one-dimensional results.

Eventually, the transient shock expansion correlated with the sudden decrease of the ram pressure when the oxygen-rich silicon shell meets the shock allows the hot bubble convection 

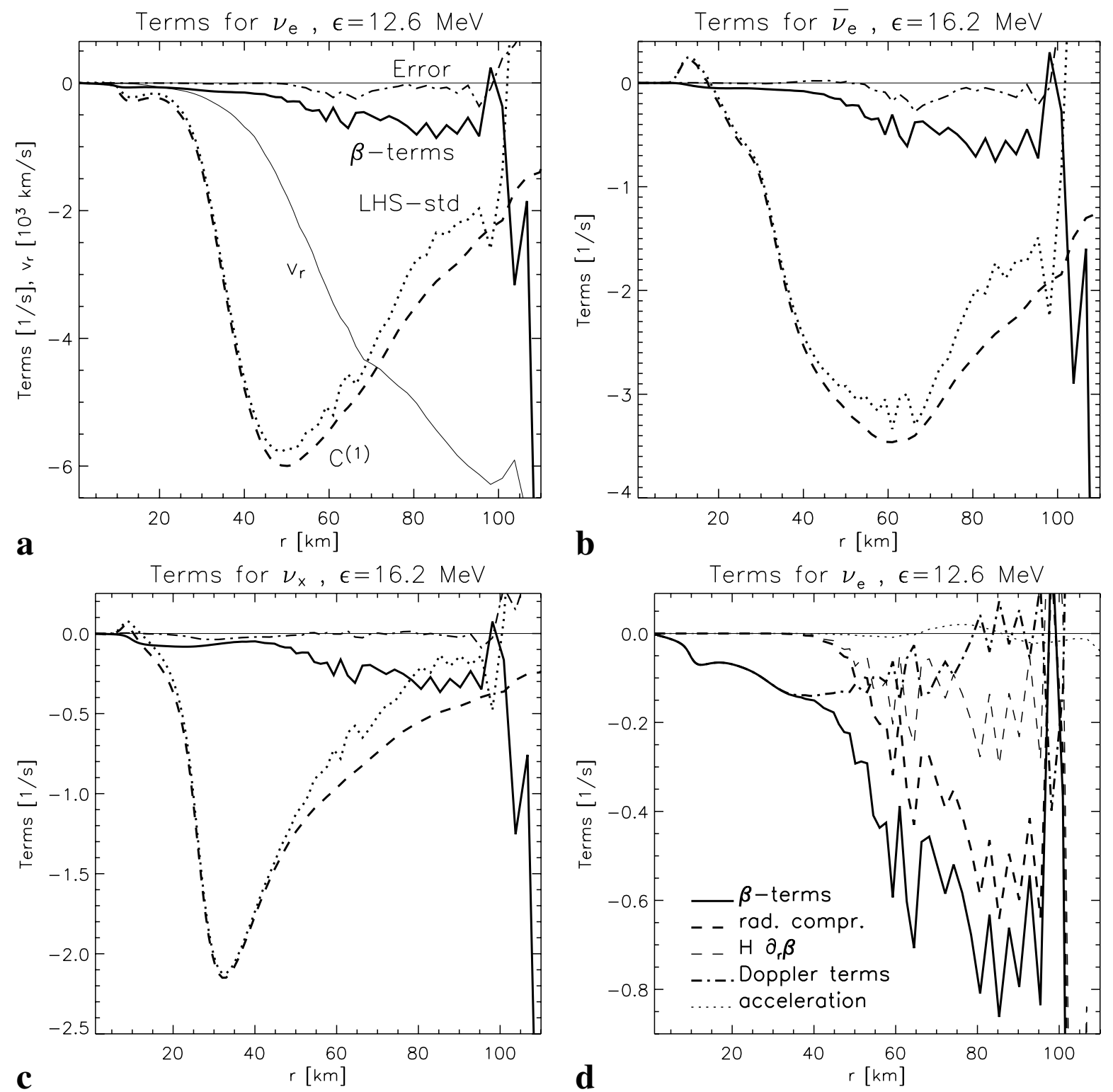

Fig. 25. Comparison of the different terms in the neutrino momentum equation (Eq. (B.14), multiplied by $m_{\text {by }} / \rho$ ) for Model s15Gio_1d.b at $t_{\mathrm{pb}}=114 \mathrm{~ms}$ for $v_{\mathrm{e}}$ (top left), $\bar{v}_{\mathrm{e}}$ (top right), and heavy-lepton neutrinos, $v_{\mathrm{x}}$ (bottom left), at the indicated energies. The different lines represent the $\beta$-terms (thick solid), the remaining terms on the LHS of Eq. (B.14) (dotted), and the source term on the RHS (dashed). We have also plotted the sum of all three (dash-dotted), which reveals minor deviations from zero as a consequence of the post-processing evaluation of the discretized gradients. Finally, the thin solid line in the upper left plots shows the velocity profile. Panel d) displays for $v_{\mathrm{e}}$ the different $\beta_{r}$-terms in the neutrino momentum equation (Eq. (B.14), multiplied by $m_{\text {by }} / \rho$ ) individually. These correspond to the physical effects of radiation compression, $H r^{-2} \partial_{r}\left(r^{2} \beta_{r}\right)$ (thick dashed) and the term $H \partial_{r} \beta_{r}$ (thin dashed), Doppler effects (thick dash-dotted), and fluid acceleration (thin dotted).

to strengthen because the accretion velocities behind the shock are reduced. At this time $(180 \mathrm{~ms})$, however, the shock has already retreated to below $100 \mathrm{~km}$, and the infall velocity is too large to be inverted by the outward acceleration of buoyant, neutrino-heated matter.

Figure $32 \mathrm{~b}$ is suitable for showing the effects arising from convection. While entropy fluctuations develop due to the convective activity after convection has become distinct about $60 \mathrm{~ms}$ after bounce, convection is too weak to noticeably perturb the entropy profile about $80 \mathrm{~ms}$ later. The perturbations grow again when convection is transiently revived after the $\mathrm{Si}-\mathrm{SiO}$ interface has passed the shock. Interestingly, the entropy perturbations persist for some time when they are advected downstream into the convectively stable cooling region (compare with Fig. 32a).

A feature visible in Fig. 32 betwen 30 and $50 \mathrm{~km}$ needs to be explained: large lateral velocities associated with matter advected downward from the HB to a convective zone in the PNS show up between 100 and $150 \mathrm{~ms}$ post-bounce. This phenomenon is caused by the periodic lateral boundary conditions applied in our simulation, which allow rings of uniform, lateral velocity to develop and to be stable. In case of reflecting 

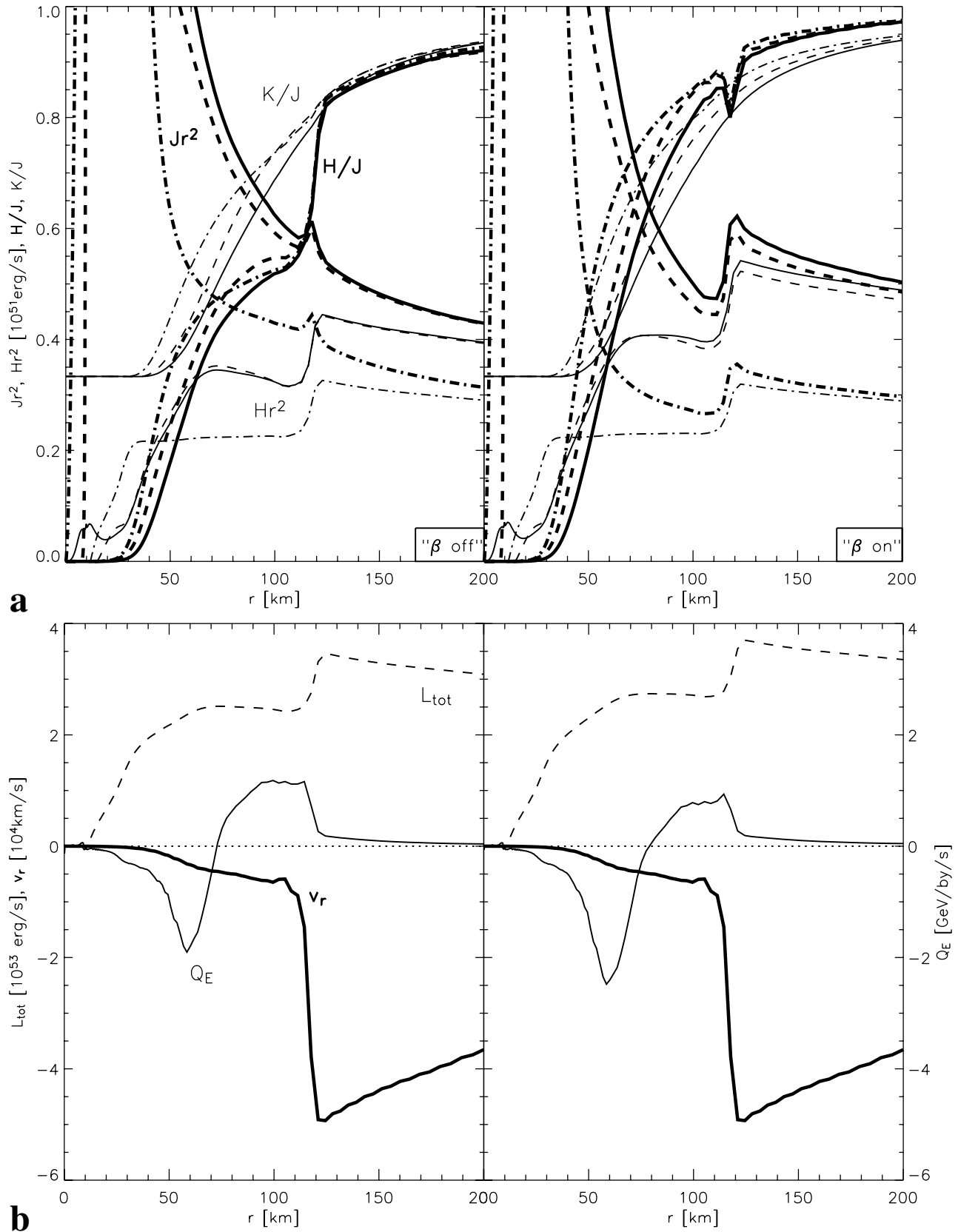

Fig. 26. a) Comparison of steady-state transport solutions without (left) and with the $\beta$-terms in the neutrino momentum equation, computed for a background profile from Model s15Gio_1d.b at $t_{\mathrm{pb}}=114 \mathrm{~ms}$. The neutrino energy density $J$ (times $r^{2}$ ), the flux factor $f_{H}=H / J$ (both with thick lines), the neutrino energy flux $H$ (times $r^{2}$ ), and the variable Eddington factor $f_{\text {ed }}=K / J$ (both thin) are given for electron neutrinos (solid), electron antineutrinos (dashed) and muon neutrinos (dash-dotted) for an observer comoving with the stellar fluid. The kink of $f_{H}$ within the shock is a numerical artifact produced by the interpolation of $J$ and $H$ between the grid zones of a staggered mesh. b) Corresponding velocity profile (thick), total neutrino luminosities (as the sum of the luminosities of neutrinos of all flavors; dashed) and the energy source terms (neutrino heating minus cooling; thin solid line).

boundary conditions such an effect would be suppressed. This reminds us that in two-dimensional simulations the chosen lateral boundary conditions determine the results to some extent. In the present case the kinetic energy stored in the rings of lateral motion is negligible compared to the energy needed to trigger an explosion and we are therefore confident that this numerical phenomenon has no macroscopic dynamical consequences.
Figure 32 shows that a second region of convective activity is present inside the PNS and persistently encompasses a layer between $10-15 \mathrm{~km}$ and about $30 \mathrm{~km}$. The layer of large lateral velocities is somewhat more extended in the radial direction than the layer of convective instability due to over- and undershooting of buoyant matter. The absence of visible entropy fluctuations in Fig. 32b is explained by the very high efficiency with which the PNS convection transports and redistributes 

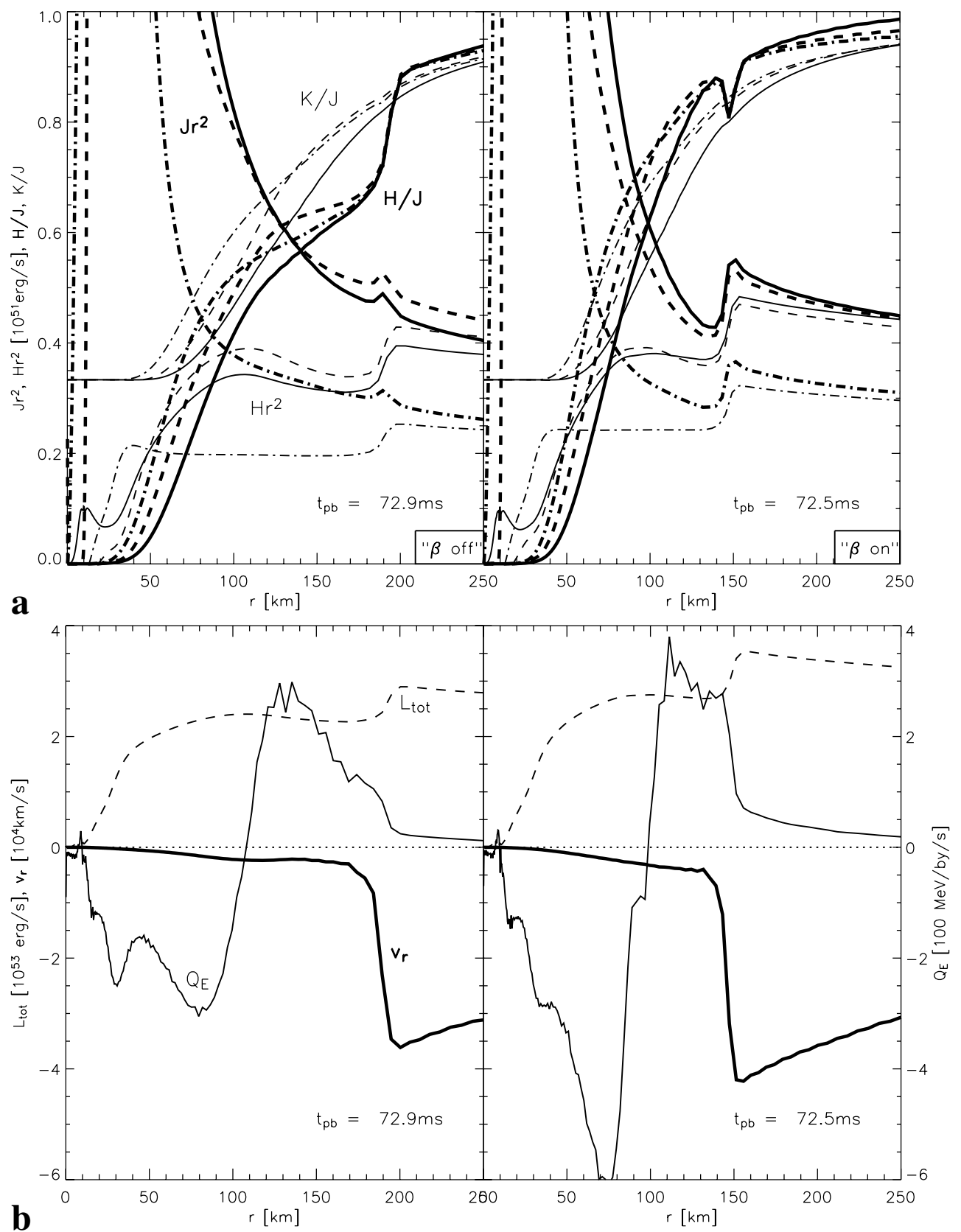

Fig. 27. a) Transport results at $t_{\mathrm{pb}}=73 \mathrm{~ms}$ for Model s15Gio_1d.a where the $\beta$-terms were dropped, and for Model s15Gio_1d.b, which was computed with all terms in the neutrino momentum equation. The neutrino energy density $J$ (times $r^{2}$ ) and the flux factor $f_{H}=H / J$ are plotted with thick lines, the neutrino energy flux $H$ (times $r^{2}$ ) and the variable Eddington factor $f_{\text {ed }}=K / J$ with thin lines for electron neutrinos (solid), electron antineutrinos (dashed) and muon neutrinos (dash-dotted). b) The corresponding profiles of velocity (thick), total luminosity of neutrinos and antineutrinos of all flavors (dashed) and the source term for energy exchange between neutrinos and stellar medium (thin solid).

entropy. Therefore the entropy gradient becomes extremely flat in this region. Despite of leading to changes of the PNS structure and affecting the neutrino emission, this convection inside the PNS has no major consequences for the NS evolution during the simulated periods of time. We will discuss these effects in greater detail in our forthcoming Paper II.

\subsection{2. s15Gio_32.a: a model with artificial explosion}

A comparison of the simulations performed for this work shows that none of our models with the full implementation of relevant physics (state-of-the-art description of neutrino-matter interactions, general relativistic gravity, variable Eddington factor closure of coupled set of neutrino moments equations and model Boltzmann equation) yields explosions, neither in spherical symmetry nor in 2D with convective processes taken into account. But as we already have seen in connection with Model s15Gio_1d.a, the omission of the $\beta_{r}$-dependent terms (but not the advection term $\beta_{r} \partial / \partial r$, which is always included) in the neutrino momentum equation (Eq. (B.14)) makes a big difference and favors explosions. Model s15Gio_32.a is the counterpart of this 1D model in two spatial dimensions and accounts for the fact that the neutrino-heated "hot bubble" region behind the shock is convectively unstable according to the 


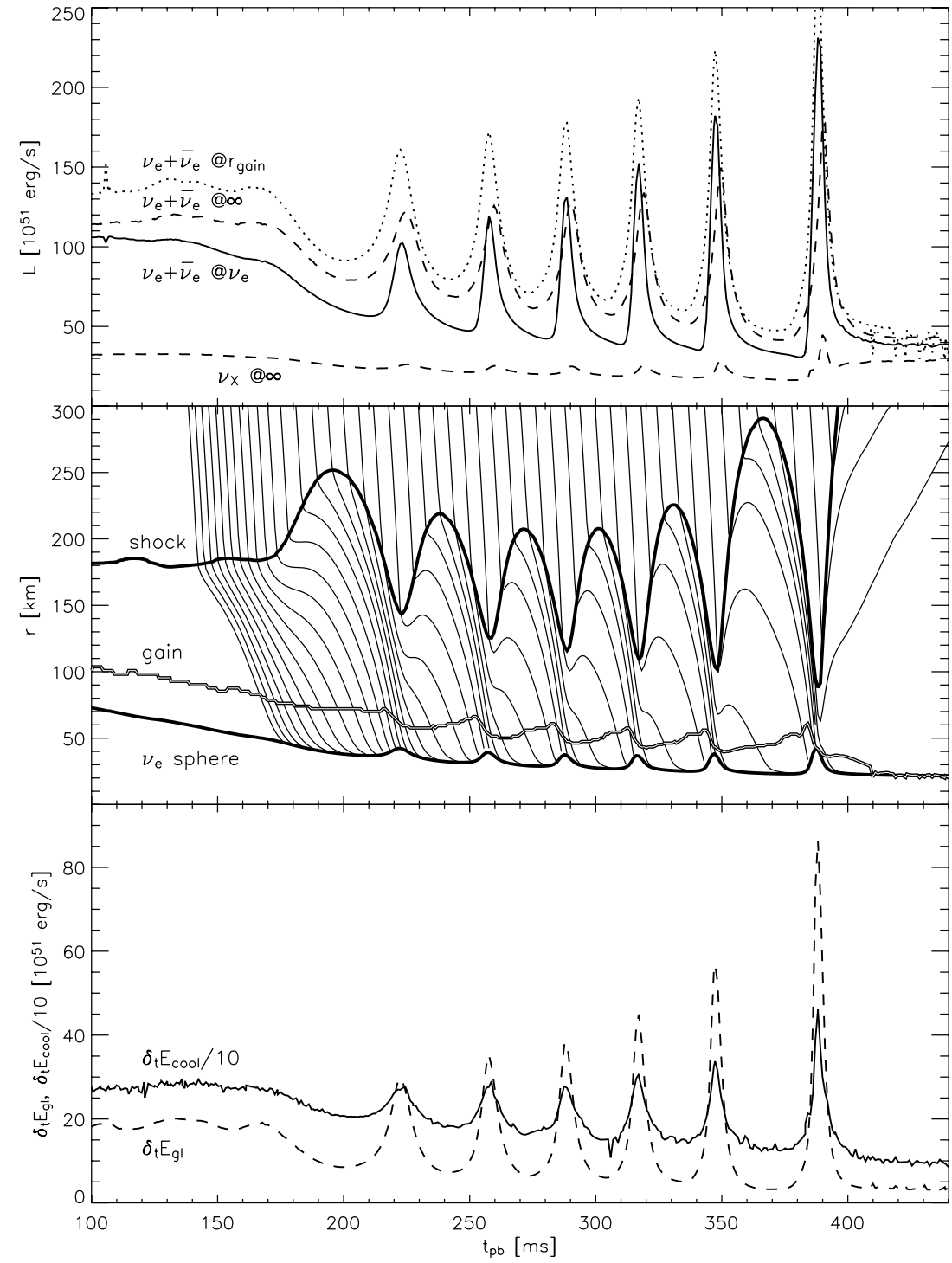

Fig. 28. Post-bounce evolution of Model s15Gio_1d.a. The upper panel shows the summed comoving-frame luminosity of electron neutrinos and antineutrinos at the neutrinosphere of $v_{\mathrm{e}}$ (solid) and at the gain radius (dotted), and this luminosity at $400 \mathrm{~km}$ for an observer at rest (dashed). The lower dashed line corresponds to the (individual) luminosity for neutrinos of $\mu$ or $\tau$ type, also for an observer at rest at $400 \mathrm{~km}$. The middle panel displays the evolution of the shock (upper thick line), gain radius, and $v_{\mathrm{e}}$-sphere (lower thick line). The thin lines represent mass shells, starting at $M=1.41 M_{\odot}$ and increasing in mass in steps of $0.002 M_{\odot}$. The lines were truncated at the $v_{\mathrm{e}}$-sphere. The lower panel depicts the total energy loss rate in neutrinos from the cooling layer (solid, divided by 10) and the net energy deposition rate by neutrinos in the gain layer (dashed). Note that here, in contrast to Fig. 14, the curves were not smoothed over time intervals.
Ledoux criterion (Fig. 30b). However the reader should keep in mind that the omission of the $\beta$-terms in Eq. (B.14) is an approximation which alters the neutrino transport in an unacceptable way. Thus, the explosion obtained in Model s15Gio_32.a has to be considered as artificial.

Note that Model s15Gio_32.a was calculated with a 2D polar grid for $r \geq 25 \mathrm{~km}$, but spherical symmetry was assumed for radii smaller than $25 \mathrm{~km}$, thus ignoring the existence of a convectively unstable layer below the neutrinosphere. However, convection inside the PNS does not have a significant influence on the explosion which develops in the discussed model; it is important for the cooling of the nascent PNS only on a timescale much longer than $100 \mathrm{~ms}$.

For continuing the simulation to late stages with acceptable requirements of CPU time we have mapped the $2 \mathrm{D}$ calculation on a 1D grid after $468 \mathrm{~ms}$ of post-bounce evolution. At this time, multi-dimensional processes (transient downflows of low-entropy matter) affect the onset of the neutrino-driven wind below $200 \mathrm{~km}$, while the immediate postshock layer is convectively only weakly unstable (Fig. 34a) and convective structures in the bulk of expanding matter between this layer and the developing wind are going to freeze out and expand essentially self-similarly. The mapping required to set the lateral velocity components as well as the corresponding momentum and kinetic energy terms to zero. Along with this change we turned on the $\beta$-terms in the neutrino momentum equation to follow the evolution of the neutrino-driven wind from the proto neutron star more realistically, and switched to the more sophisticated treatment of nuclear statistical equilibrium at low densities by a 4-species Saha solver as described in Sect. 2.4. The latter replacement was necessary because the previous approximative description of NSE in the low-density EoS of Rampp \& Janka (2002, Appendix B) did not solve the Saha equations but described nuclear dissociation and recombination by temperature-dependent composition changes which do not guarantee entropy conservation and thus cause numerical artifacts during the later phases of wind evolution.

Note that the steepening density gradient at the PNS surface with ongoing time made it necessary to refine our Eulerian grid in this region. Rezoning was done at $206 \mathrm{~ms}, 468 \mathrm{~ms}$, and $759 \mathrm{~ms}$. We should also mention that Model s15Gio_32.a did only take into account $\mathrm{Si}$ burning, but not $\mathrm{C}, \mathrm{O}, \mathrm{Mg}$, 

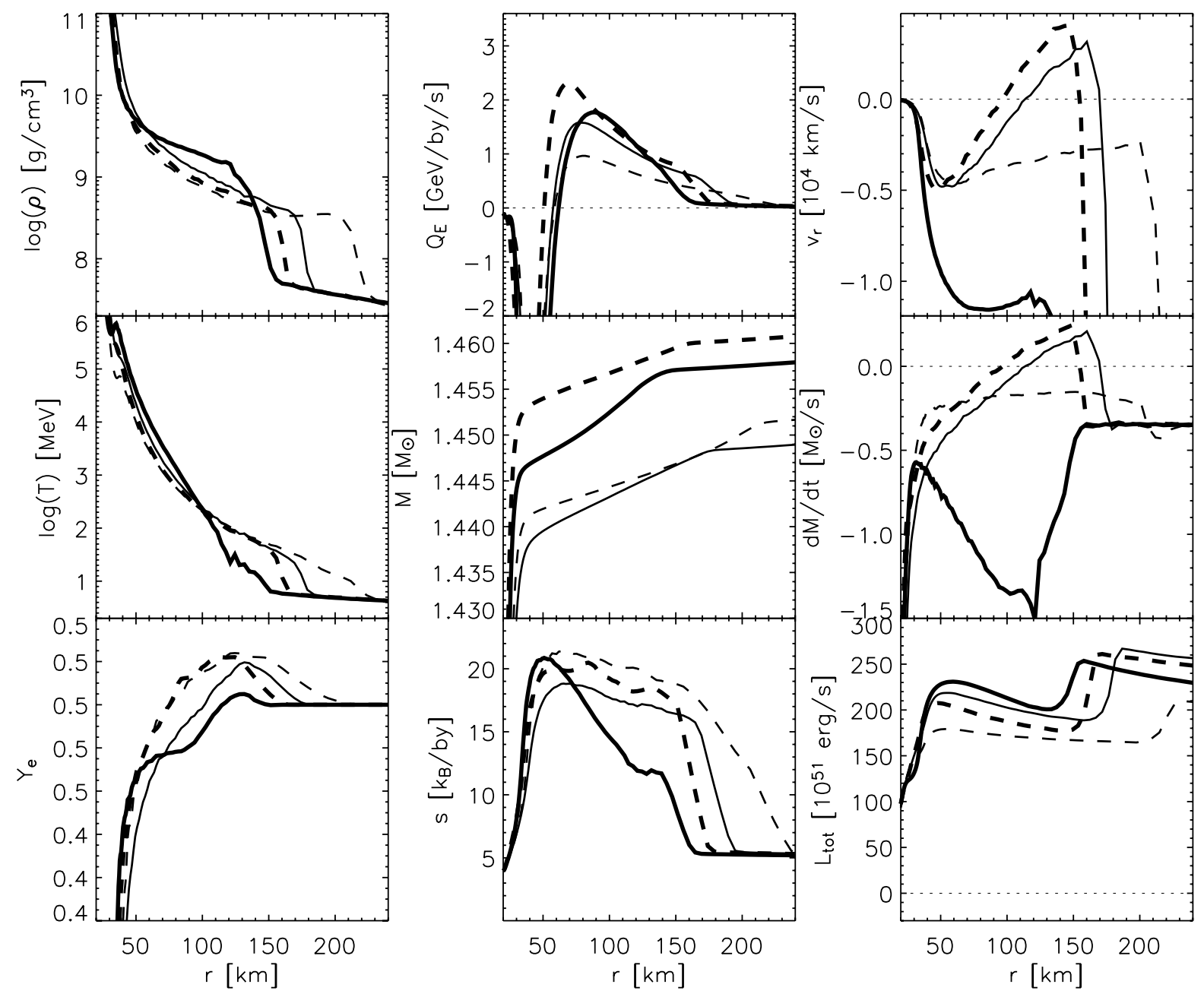

Fig. 29. Radial structure of Model s15Gio_1d.a at post-bounce times of $228.6 \mathrm{~ms}$ (solid), $236.4 \mathrm{~ms}$ (thin dashed), 254.4ms (thick solid), and $262.6 \mathrm{~ms}$ (thick dashed). At the first and last time, the post-shock velocities reach their maximum positive values, at the second time the shock reaches its local maximum, and at the third time the post-shock velocities reach their minimum negative values. At $254 \mathrm{~ms}$ the maximum cooling rate reaches $Q_{\mathrm{E}}=-30.1 \mathrm{GeV} / \mathrm{s}$ per baryon.

and Ne burning as described in Rampp \& Janka (2002). A post-processing estimate, however, showed that oxygen burning would have contributed less than $0.011 \times 10^{51} \mathrm{erg}$ to the explosion energy, while the ignition conditions for the other burning processes were not reached in our model.

Evolution. Model s15Gio_32.a develops powerful convection. Like in Model s15Gio_32.b, convective activity in the gain layer starts at about $60 \mathrm{~ms}$ after bounce (see Fig. 35). However, in this model neutrino heating is stronger (the heating integral is higher roughly by a factor of 2, cf. $\delta_{t} E_{\mathrm{gl}}$ in Fig. 14) due to the omission of the $\beta$-terms, leading to a steeper entropy gradient, which in turn means a stronger destabilization of the gain layer. Furthermore, the stronger heating is sufficient to prevent the shock contraction seen in Model s15Gio_32.b after $70 \mathrm{~ms}$ post-bounce (see Fig. 14). As a consequence the convective region between gain radius and shock remains extended and the convective cells can grow and merge to larger structures, leading to the largest scale possible on the grid for the boundary conditions imposed in our simulation (i.e. a wedge of $\pm 43.2^{\circ}$ around the equator with periodic boundary conditions): after about $150 \mathrm{~ms}$ postbounce one big convective cell with a rising bubble and one downflow has formed and transports energy efficiently to the shock (Fig. 35). Convection in this model becomes strong enough to push the shock farther out; the gain layer, and therefore the convective region, continuously expand after convection has set in (Figs. 13, 36). Finally, convection supports an explosion, although the corresponding 1D model, s15Gio_1d.a, did not explode at such an early time.

The onset of the explosion occurs when the infalling oxygen-rich silicon shell arrives at the shock at $260 \mathrm{~km}$ and $150 \mathrm{~ms}$ after bounce. The interface between the silicon layer and the O-rich silicon shell is characterized by a steep entropy jump and the preshock density drops to a much lower value. This leads to a sudden decrease of the mass accretion rate and thus of the ram pressure. The shock reacts to that by expansion and rapid acceleration. Although the explosion starts at this moment, the existence of the composition interface may not be crucial for the explosion of Model s15Gio_32.a. Favorable conditions for an explosion can be seen even before the composition interface has reached the shock: for 


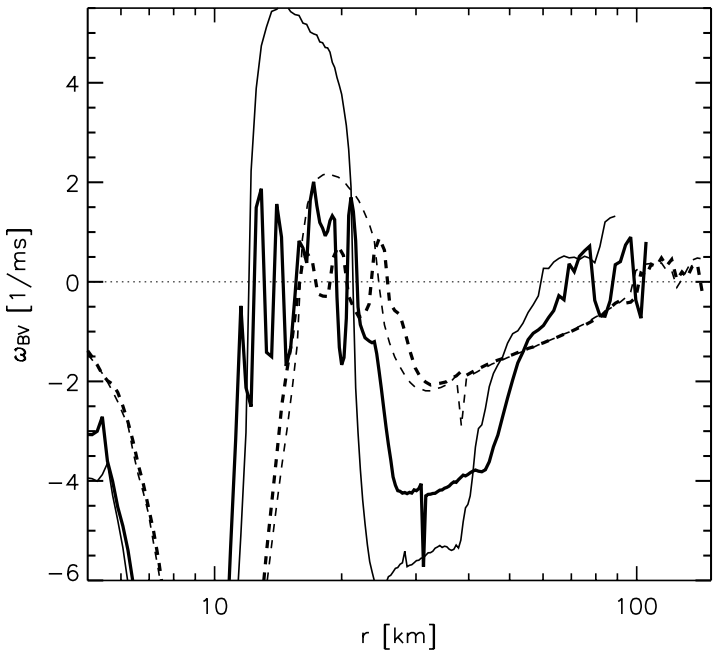

$\mathbf{a}$

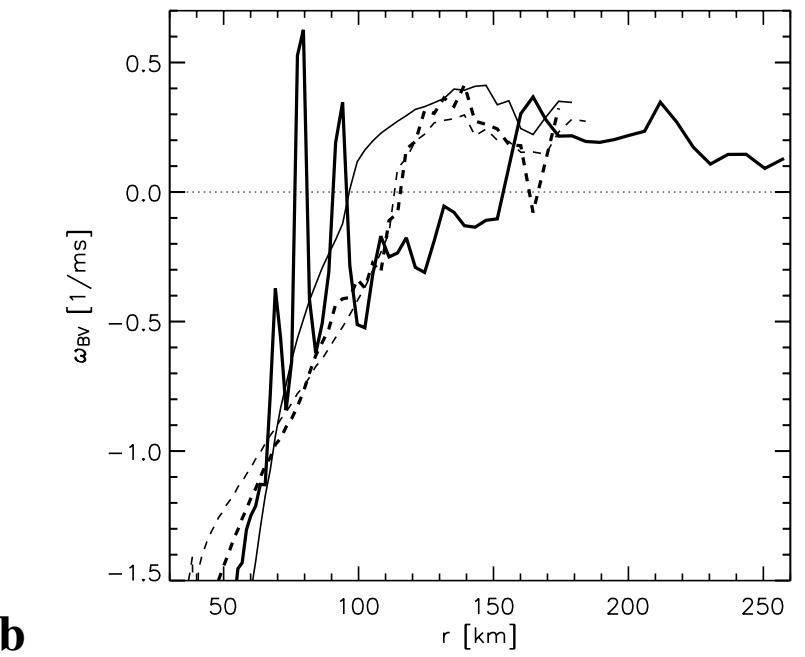

Fig. 30. a) Brunt-Väisälä frequency as defined in Sect. 2.3 .2 at the post-bounce times of $72.3 \mathrm{~ms}$ (dashed) and $181.4 \mathrm{~ms}$ (solid) for Model s15Gio_1d.b (thin) and s15Gio_32.b (thick). The lines are plotted only downstream of the shock. Positive values indicate instability. For the 2D model the evaluation was performed with laterally averaged quantities. b) Same as a), but for models s15Gio_1d.a (thin) and s15Gio_32.a (thick) at post-bounce times of $72.2 \mathrm{~ms}$ (dashed) and $150.1 \mathrm{~ms}$ (solid).

one the shock is already expanding (Fig. 36). Second, the mass contained in the gain layer remains constant between 70 and $170 \mathrm{~ms}$ after bounce in contrast to all other simulations, including Model s15Gio_1d.a, where $M_{\mathrm{gl}}$ is always decreasing, see Fig. 37. (Note that the difference in $M_{\mathrm{gl}}$ between Models s15Gio_32.a and s15Gio_1d.a during the first $70 \mathrm{~ms}$ after bounce arises from the omission of the $v v \leftrightarrow v v$ rates in the latter model). Third, the matter in the gain layer is accumulating energy: the whole gain layer is continuously getting closer to become gravitationally unbound (Fig. 37) and the energy contained in grid zones with positive local specific binding energy ("explosion energy"; Eq. (29)) already starts growing after $120 \mathrm{~ms}$ post-bounce (Fig. 38). Also, looking at the profiles at $t_{\mathrm{pb}}=150 \mathrm{~ms}$ in Fig. 39, one can see that the jump in entropy and density has not yet arrived at the shock, but that the average radial velocities behind the shock have almost become positive. All these facts suggest that the convective overturn in the gain layer has been successful in transporting energy from the gain radius to the shock and thus has increased the heating efficiency.

Shortly after the launch of the outgoing SN shock the density in the region between dense post-shock shell and PNS drops (Fig. 40) and the gain radius retreats to a position just outside the neutrinosphere, which it reaches about $300 \mathrm{~ms}$ after bounce (Fig. 36). The onset of the neutrino-driven wind after the start of the explosion is visible in Fig. 40 as a rapid (in our simulation spherically symmetric), baryonic outflow with velocities up to $\sim 5 \times 10^{9} \mathrm{~cm} / \mathrm{s}$. It develops after $\sim 500 \mathrm{~ms}$ post-bounce (see also Fig. 36 ) and catches up with the slower and denser shell of early SN ejecta. In the deceleration region a reverse shock (wind termination shock) develops (Janka \& Müller 1995; Tomàs et al. 2004) which is a very prominent feature in the density, velocity, temperature, and entropy profiles of Fig. 40 after $\sim 600 \mathrm{~ms}$ post-bounce (see also Fig. 36).

Figure $34 \mathrm{a}$ shows the convective activity during the 2D phase of the simulation. While convection in the PNS is inhibited to develop despite of convective instability because we calculate with a spherically symmetric grid below $25 \mathrm{~km}$, large parts of the gain layer are unstable and show convective motions (Fig. 35). On the other hand we see that in the matter swept up by the expanding shock convective instability is so weak (the condition $\omega_{\mathrm{BV}}<30 / \mathrm{s}$ applies) that convective activity between about 1000 and $3000 \mathrm{~km}$ does not set in. Around $320 \mathrm{~ms}$ after bounce the downflows of mass to the PNS cease (see also Fig. 34b) and the neutrino-driven baryonic outflow begins to develop (Fig. 36). However, a massive fallback in the form of several downflows occurs between $390 \mathrm{~ms}$ and $440 \mathrm{~ms}$ after bounce and destroys this neutrino-driven "wind". The wind begins to recover again afterwards (Fig. 36). In Fig. 34b we see that the mass inflow (at $100 \mathrm{~km}$ ) becomes almost equal to the mass outflow after the onset of explosion, meaning that the net mass flow is small compared to the overturn. Consistent with Fig. 34a, the outflow dominates between $300 \mathrm{~ms}$ and $390 \mathrm{~ms}$ post-bounce and after $440 \mathrm{~ms}$ post-bounce, while the transient downflows between $390 \mathrm{~ms}$ and $440 \mathrm{~ms}$ lead to strong overturn activity also in the vicinity of the nascent NS.

Explosion energy. To calculate the explosion energy at a given time, we add up the local specific binding (LSB) energy $\varepsilon_{\text {bind }}^{\text {shell }}$, defined in Eq. (27), for all grid zones between neutrinosphere and shock where this energy and the radial velocity are both positive:

$E_{\text {expl }}=\sum_{r, \vartheta \text {,cond }} \varepsilon_{\text {bind }}^{\text {shell }}(r, \vartheta) \rho(r, \vartheta) \Delta V(r, \vartheta)$.

Note that the gravitational potential $\Phi_{1 \mathrm{D}}^{\text {Newt, enclosed }}(r)$ used to calculate the LSB energy was calculated assuming a spherically symmetric mass distribution (2D effects are only caused by convective mass motions and the associated density fluctuations are modest and therefore play a minor role for the gravitational potential).

The explosion energy as a function of time is shown in Fig. 38. Its increase with time has several reasons: the 


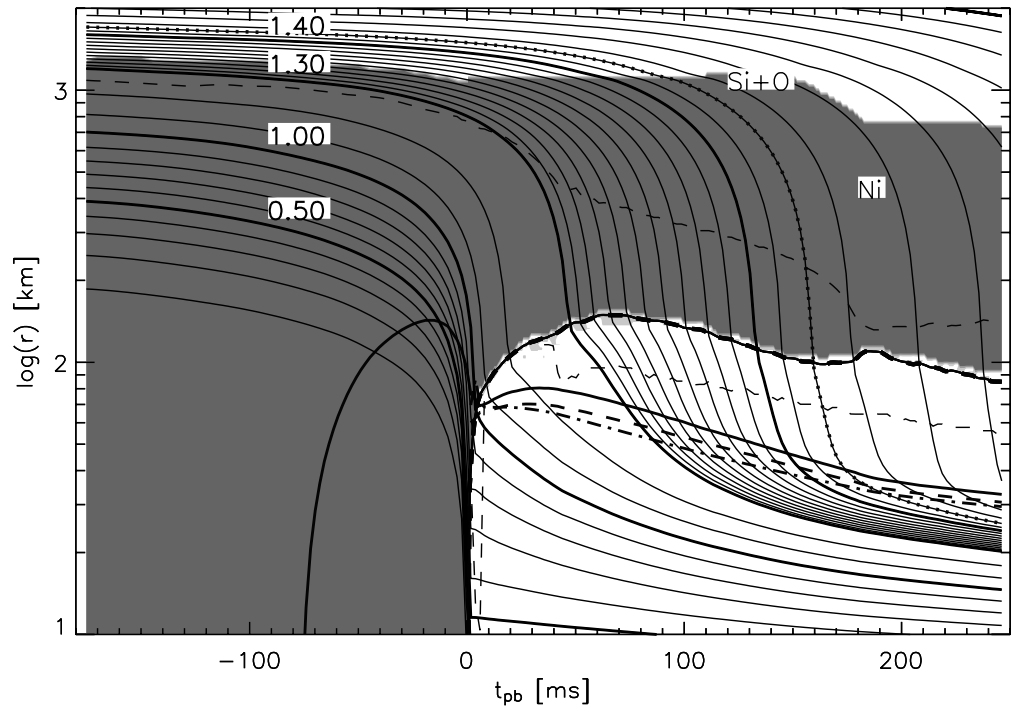

Fig. 31. "Mass shell" trajectories for Model s15Gio_32.b. The shells plotted with bold solid lines are labelled by the corresponding enclosed masses. The plot also shows the transport neutrinospheres for $v_{\mathrm{e}}$ (thick solid), $\bar{v}_{\mathrm{e}}$ (thick dashed), and $v_{\mathrm{x}}$ (thick dash-dotted), the mass shell at which the silicon shell becomes oxygen-rich (knotted solid line, at $1.42 M_{\odot}$ ), and the angle-averaged shock radius (solid line with dashes). Further, we have marked the regions which contain a mass fraction of more than $60 \%$ in Fe-group elements (dark shaded) and of more than $30 \%$ in ${ }^{4} \mathrm{He}$ (light shaded, visible in the vicinity of the shock). Finally, the inner thin dashed line marks the gain radius, while the outer one marks the interface between the regimes of the low-density and high-density EoSs $\left(\rho=6 \times 10^{7} \mathrm{~g} \mathrm{~cm}^{-3}\right)$. deposition of energy by neutrinos is the dominant contribution during the early stages of the explosion after it has taken the LSB energy in the gain layer to a value close to zero even before rapid expansion has begun and the explosion has set in (Fig. 37). When the shock reaches a radius of about $400 \mathrm{~km}$ and expands farther, the post-shock matter cools sufficiently that neutrons and protons start recombining to ${ }^{4} \mathrm{He}$ (or matter accreted through the shock is not sufficiently heated to dissociate to free nucleons), which releases/saves about 7.5 MeV per baryon and causes the energy in the gain layer to become positive. Subsequently helium recombines further to Fe-group elements, releasing another $\sim 1.3 \mathrm{MeV}$ per baryon. When the shock reaches about $800 \mathrm{~km}$ (corresponding to an enclosed mass of 1.45-1.46 $M_{\odot}$ ), the material accreted by the shock is no longer dissociated to later recombine, but instead, silicon burns in the shock to ${ }^{56} \mathrm{Ni}$. This process, however, ends when the shock reaches about $1700 \mathrm{~km}\left(1.527 M_{\odot}\right)$ and does not heat the stellar gas to Si-burning temperatures any more. At late times, the explosion energy is increased by the energy input from the baryonic wind which is driven by neutrino energy deposition just outside of the neutrinosphere (starting at $t \gtrsim 300 \mathrm{~ms}$ after bounce). The wind material initially also fully recombines, releasing additional nuclear binding energy, but with decreasing densities and increasing entropy the recombination becomes incomplete and a large number of $\alpha$-particles are left ( $\alpha$-rich freeze-out). The time evolution of the composition in the infalling and exploding layers is indicated by the grey shadings in Fig. 36.

On the other hand, the shock also loses energy by lifting accreted material of the outer layers in the gravitational potential of the star.

The explosion energy in Fig. 38 is already positive when the interface between Si-layer and O-rich $\mathrm{Si}$-shell passes the shock at $150 \mathrm{~ms}$. We then notice a steep rise after the onset of the explosion due to neutrino heating, nucleon recombination, and burning. At $300 \mathrm{~ms}$ post-bounce burning ceases and the energy increase becomes less steep. Finally, around $400 \mathrm{~ms}$, the $v$-driven wind sets in, which contributes energy at a much smaller rate: the energy increases only very slowly. The transient little drop in the explosion energy between $468 \mathrm{~ms}$ and $500 \mathrm{~ms}$ results from the mapping of the model from a 2D to a $1 \mathrm{D}$ grid.

At the end of our simulation, the shock has reached an enclosed mass of $1.9 M_{\odot}$. From Fig. 10 we can thus say that the shock will still lose an energy of $0.16 \mathrm{FOE}(\mathrm{FOE}=$ ten to the fifty-one erg) for unbinding the outer layers of the star. The final value of the explosion energy will also be changed by further input from the $v$-driven wind, and possibly by fallback of material onto the PNS. Assuming that wind power and gravitational binding energy of the outer layers roughly compensate each other, a value of $0.5 \mathrm{FOE}$ seems to be a good estimate for the final explosion energy of our model. This is comparable to the energy that has been estimated for SN1999br (Hamuy 2003) and must certainly be considered as "weak" explosion.

The neutron star left behind by the explosion has an initial baryonic mass of about $1.4 M_{\odot}$. Figure 40 shows that it is $1.41 M_{\odot}$ at $800 \mathrm{~ms}$ after bounce, which can decrease by some $0.01 M_{\odot}$ due to losses in the neutrino-driven wind, and later increase due to possible fallback.

Mass and composition of ejecta. The discussed explosion model reveals interesting properties also with respect to the neutron-to-proton ratio in the ejecta. Since we did not carry along marker particles in our Eulerian 2D simulation, the $Y_{\mathrm{e}}$ distribution of the ejecta was evaluated in a post-processing step: to determine the total amount of matter that is ejected as function of $Y_{\mathrm{e}}$, we integrate over time the flux of mass (for given value of $Y_{\mathrm{e}}$ ) through a surface of fixed radius $r_{0}$, resulting in the expression

$$
\begin{aligned}
& M\left(Y_{i}<Y_{\mathrm{e}} \leq Y_{i+1}\right)=\frac{2}{\cos \left(\vartheta_{\min }\right)-\cos \left(\vartheta_{\max }\right)} \\
& \times \sum_{n} \sum_{\vartheta, \text { cond }} \Delta A\left(r_{0}, \vartheta\right) \cdot v_{r}\left(r_{0}, \vartheta, t_{n}\right) \cdot \rho\left(r_{0}, \vartheta, t_{n}\right) \cdot\left(t_{n+1}-t_{n}\right)
\end{aligned}
$$

Here, $\Delta A=2 \pi r_{0}^{2}\left(\cos \vartheta_{j+1}-\cos \vartheta_{j}\right)$ is the cell surface through which the matter flows and the factor in front of the sum renormalizes the result from the wedge used in our simulation 
a

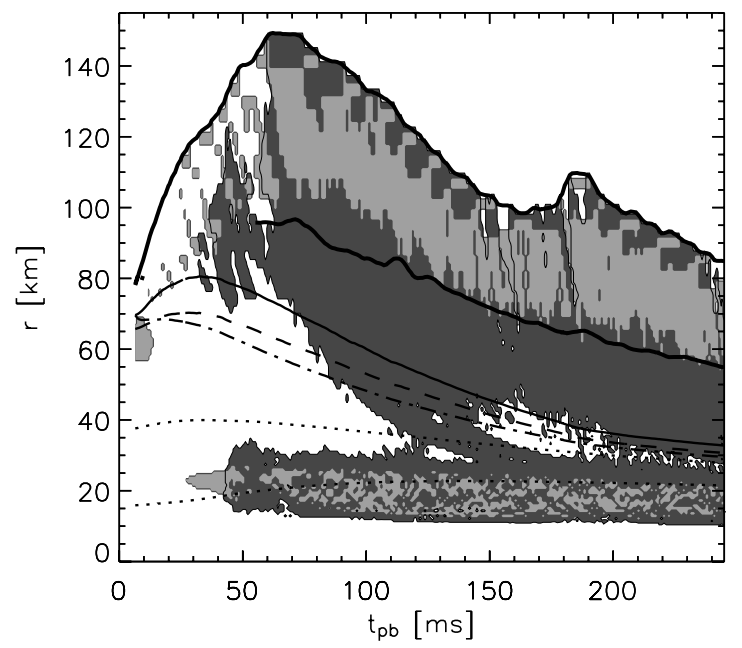

b

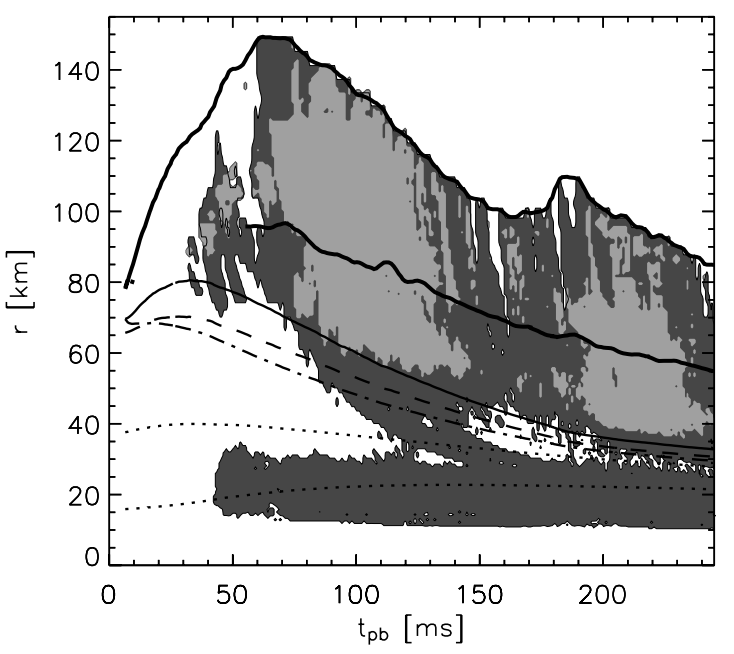

Fig. 32. a) Convective regions for Model s15Gio_32.b. Dark shading indicates regions where the lateral velocities reach more than $700 \mathrm{~km} \mathrm{~s}^{-1}$, light shading regions where the stability criterion described in Sect. 2.3.2 predicts instability. The contour lines correspond to the boundaries of the dark-shaded regions. The evaluation was performed only downstream of the shock (upper thick solid line) with polar averages of the variables. The lower thick solid line depicts the gain radius. The thin lines depict the neutrinospheres for $v_{\mathrm{e}}$ (solid), $\bar{v}_{\mathrm{e}}$ (dashed), and $v_{\mathrm{x}}$ (dash-dotted). Finally, the dotted lines show where the densities are $10^{12} \mathrm{~g} \mathrm{~cm}^{-3}$ (upper line) and $10^{13} \mathrm{~g} \mathrm{~cm}^{-3}$ (lower line). b) Same as a) but with the light shading marking regions where the standard deviation of entropy variations (defined in analogy to Eq. (24)) is $\sigma_{s}>0.02$.

to the whole star. The first sum accomplishes the integration over time, while the second sum integrates over those angular bins where the following condition is fulfilled: $v_{r}\left(r_{0}, \vartheta, t_{n}\right)>$ $0 \wedge Y_{i}<Y_{\mathrm{e}}\left(r_{0}, \vartheta, t_{n}\right) \leq Y_{i+1}$ for a given bin $\left[Y_{i}, Y_{i+1}\right]$.

Choosing a suitable value for $r_{0}$ for this evaluation is not a trivial task: on the one hand, it should be as large as possible so that the matter advected through the surface at $r_{0}$ is no longer affected by neutrino processes and thus $Y_{\mathrm{e}}$ has reached its terminal value. On the other hand, the advection of material on our Eulerian grid and the associated mixing of matter tends to destroy small patches with extreme values of $Y_{\mathrm{e}}$. Therefore
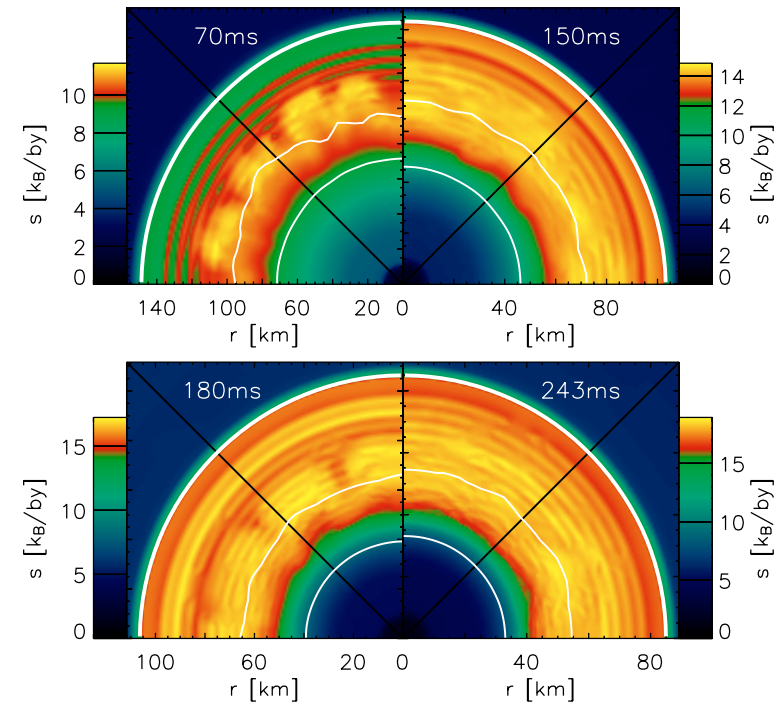

$\mathbf{a}$
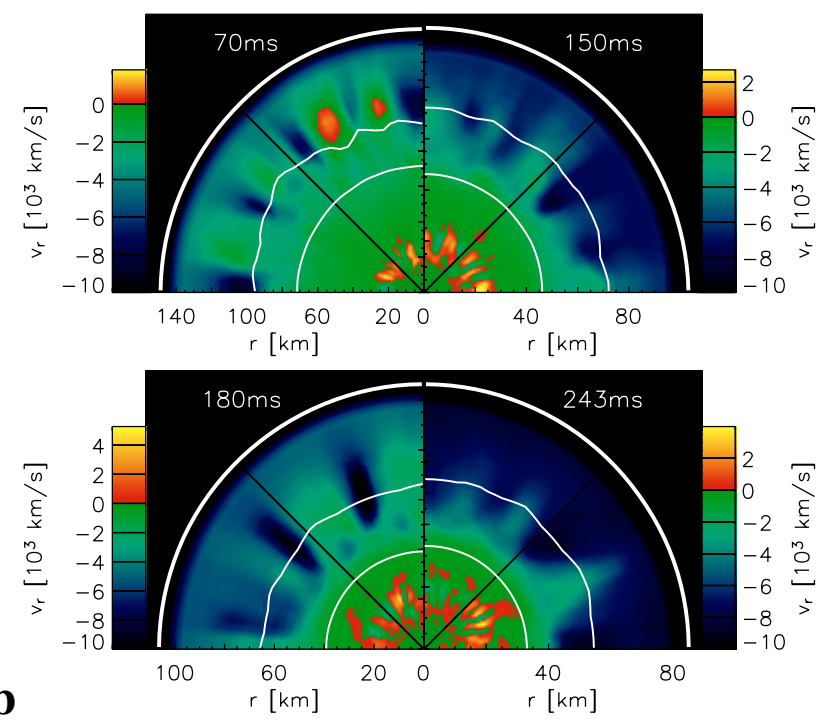

Fig. 33. Snapshots of Model s15Gio_32.b with convection in the "hot bubble" region. The white lines mark the shock position (thick), the gain radius (outer thin), and the $v_{\mathrm{e}}$-sphere (inner thin). The solid black line denotes the equatorial plane of the polar grid used in the simulation. a) These panels show colour coded the entropy $s$. b) All panels show colour coded the radial velocity $v_{r}$.

we would like to choose $r_{0}$ as small as possible to keep this numerical diffusivity as low as possible.

The results shown in Fig. 41 were obtained with $r_{0}=$ $350 \mathrm{~km}$. The time resolution of the processed data lies between one and two ms, the post-processing for Fig. 41 has ended at $t_{\mathrm{pb}}=468 \mathrm{~ms}$. To estimate the reliability of our analysis, we tested the differences caused by moving $r_{0}$ to $300 \mathrm{~km}$ (shift indicated by dark shading in Fig. 41) or $400 \mathrm{~km}$ (light shading). In the latter case, the advection between grid zones has partly "washed away" small regions of, in particular, low $Y_{\mathrm{e}}$, while in the former case we miss some neutrino processing of matter at radii between $300 \mathrm{~km}$ and $350 \mathrm{~km}$, which has the tendency of increasing $Y_{\mathrm{e}}$. The differences between the three values of $r_{0}$ can be interpreted as error bars. The integrated masses in the three cases with $r_{0}=350,300$, and $400 \mathrm{~km}$ are $M_{\mathrm{tot}}=0.0267$, 0.0273 , and $0.0274 M_{\odot}$, respectively. 

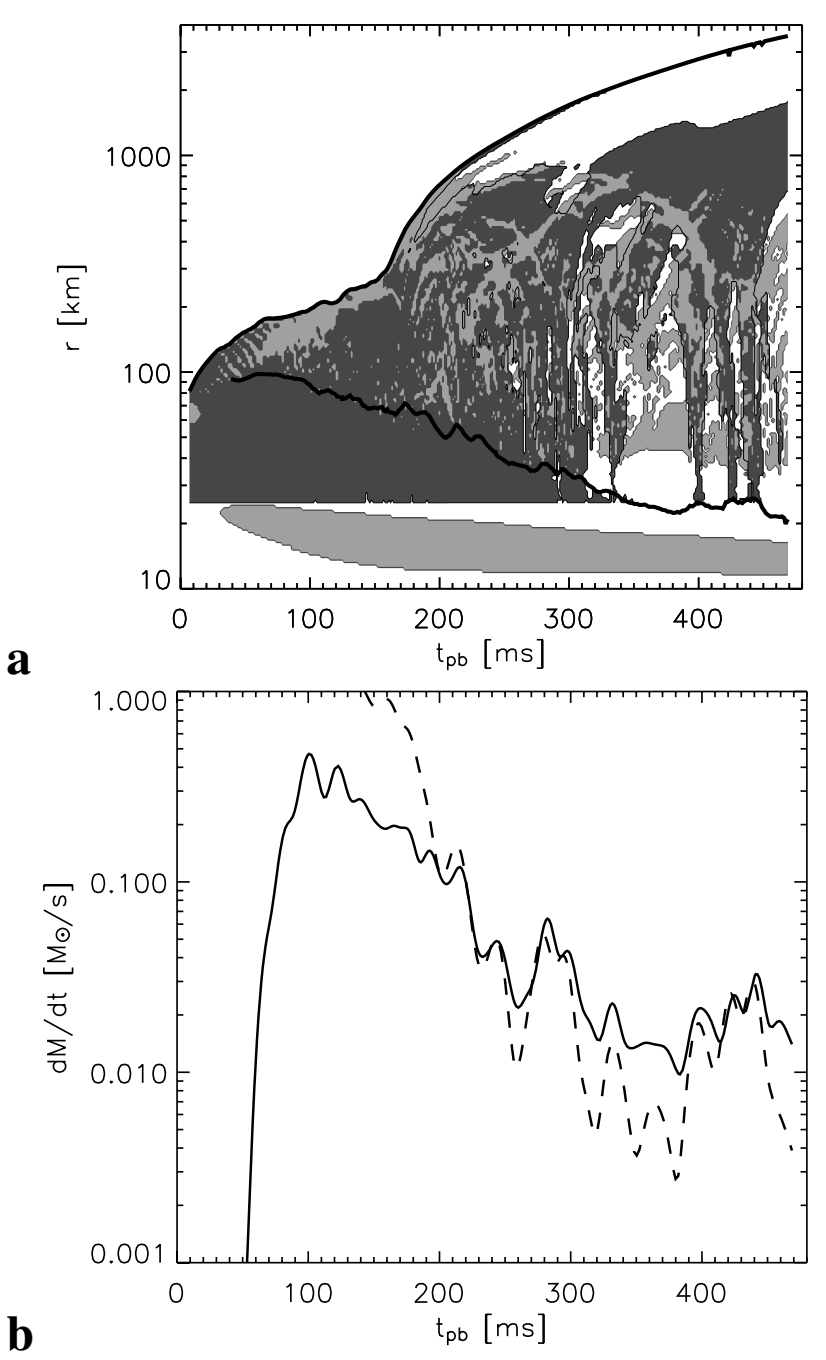

Fig. 34. a) Convective regions for Model s15Gio_32.a. Dark shading indicates where the mass inflow rate (evaluated for zones where $v_{r}<$ 0 ) exceeds $10^{-2} M_{\odot} / \mathrm{s}$. Light shading denotes regions where $\omega_{\mathrm{BV}}>$ 30/s (see Sect. 2.3.2 for the definition of the stability criterion). The contour lines mark the boundaries of the dark-shaded regions. The evaluation was performed only downstream of the shock (upper thick solid line) with polar averages of the variables. The lower thick solid line corresponds to the gain radius. Note that the core of this model inside of $25 \mathrm{~km}$ was computed in spherical symmetry. b) The solid line represents the mass outflow rate (evaluated for zones where $v_{r}>0$ ) at $r=100 \mathrm{~km}$ versus time. The dashed line shows the corresponding mass inflow rate. The lines were smoothed over time intervals of $5 \mathrm{~ms}$.

Another problem is given by material that falls back through the surface at $r_{0}$, and then reemerges with a different value of $Y_{\mathrm{e}}$; this material is counted twice in our analysis. For example, in Fig. 36 we can see that between $400 \mathrm{~ms}$ and $425 \mathrm{~ms}$, some material, about $10^{-3} M_{\odot}$, temporarily falls back below the radius of $350 \mathrm{~km}$. The amount of such double processed material, however, is small compared to the total integrated mass. Also note that the distribution of neutrino processed ejecta of Fig. 41 is not the final one because it is based on an analysis of the explosion only until $468 \mathrm{~ms}$, excluding the subsequent neutrino-driven wind. For $Y_{\mathrm{e}}<0.5$, however, the plot presents upper limits of the ejected mass at early times
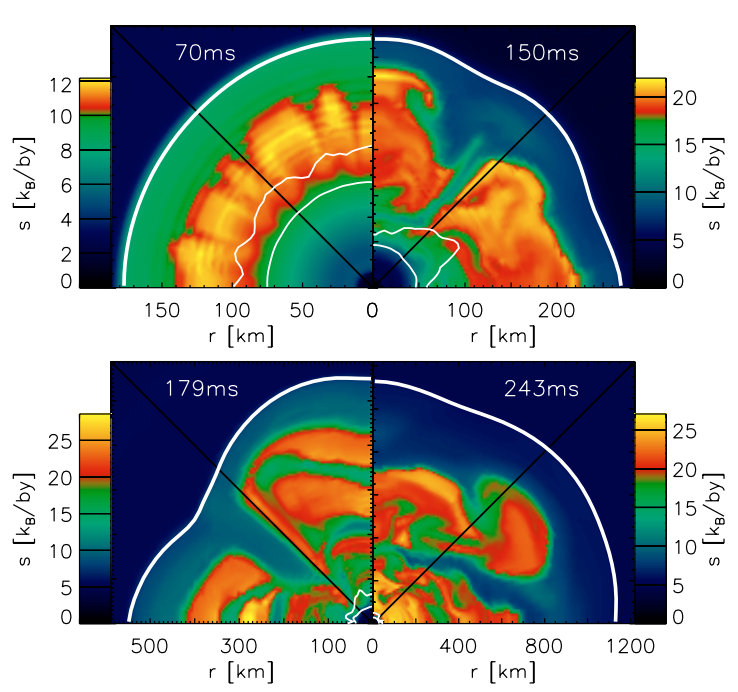

$\mathbf{a}$
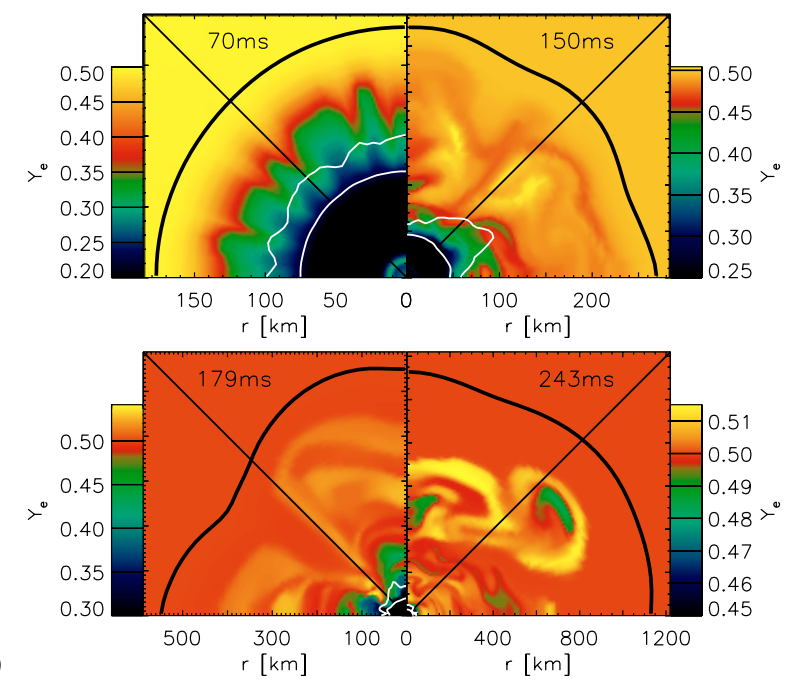

Fig. 35. 2D snapshots of Model s15Gio_32.a for four different postbounce times. a) shows the entropy $s$; b) shows the electron fraction $Y_{\mathrm{e}}$. The diagonal black lines mark the equator of the polar grid, the horizontal axis corresponds to $\cos (\vartheta)=-0.67$, the perpendicular axis to $\cos (\vartheta)=+0.67$. The shock radius is indicated by a thick black or white line, and the gain radius and $v_{\mathrm{e}}$-sphere by thin white lines.

of the explosion, because the following ejecta have $Y_{\mathrm{e}}>0.5$ (although at very late times the neutrino-driven wind might become neutron-rich again).

The production of proton-rich ejecta $\left(Y_{\mathrm{e}}>0.5\right)$ during the early phase of the supernova explosion seems to be a generic result of models with an elaborate spectral treatment of the neutrino transport either by solving the Boltzmann equation (Liebendörfer et al. 2003; Thielemann et al. 2003; Fröhlich et al. 2004) or by the variable Eddington factor closure of the moments equations used in this work (see also Pruet et al. 2005). According to Hoffman et al. (1996) the amount of supernova ejecta with $Y_{\mathrm{e}} \lesssim 0.47$ must be less than about $10^{-4} M_{\odot}$ per event in order to avoid overproduction of closed neutron shell $(N=50)$ nuclei, for example ${ }^{88} \mathrm{Sr},{ }^{89} \mathrm{Y}$, and ${ }^{90} \mathrm{Zr}$, compared to observed galactic abundances of these elements. Our explosion model fulfills this constraint (Fig. 41; possible latetime ejection of $Y_{\mathrm{e}}<0.5$ material in the neutrino-driven wind is 


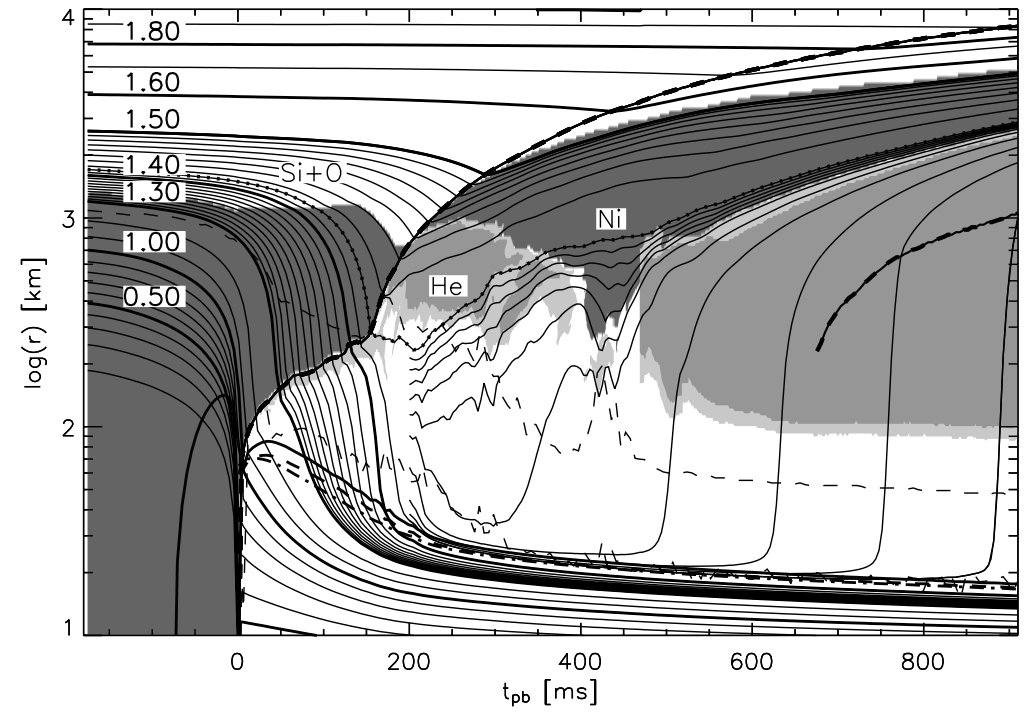

Fig. 36. "Mass shell" trajectories for the exploding 2D Model s15Gio_32.a. "Shells" plotted by bold lines are labelled with their corresponding enclosed masses. Also shown are the transport neutrinospheres for $v_{\mathrm{e}}$ (thick solid), $\bar{v}_{\mathrm{e}}$ (thick dashed), and $v_{\mathrm{x}}$ (thick dash-dotted), the interface between the silicon shell and the oxygen-rich silicon shell (knotted solid line, at $1.42 M_{\odot}$ ), and the shock and reverse shock or wind termination shock (thick solid lines with dashes). Moreover, the regions are marked which contain a mass fraction of more than $60 \%$ of $\mathrm{Fe}$ group elements (dark shaded) or more than $60 \%$ of ${ }^{4} \mathrm{He}$ (shaded). Light shading indicates other regions which have a mass fraction of more than $30 \%$ of ${ }^{4} \mathrm{He}$. The lower dashed line denotes the gain radius and the upper one marks the interface between the low-density and highdensity EoSs (i.e. this line corresponds to $\rho=6 \times$ $\left.10^{7} \mathrm{~g} \mathrm{~cm}^{-3}\right)$.

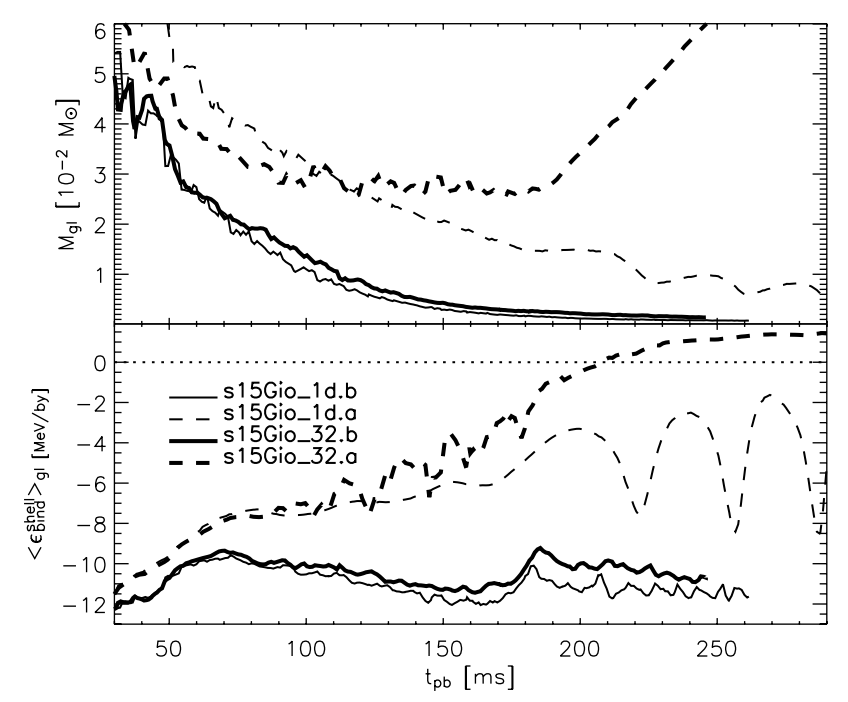

Fig. 37. Time evolution of the total mass in the gain layer and the average local specific binding energy in the gain layer for four models, including the exploding Model s15Gio_32.a.

unlikely to be sufficiently massive to be in conflict with the observations) in contrast to previous models with partly crude or simple approximations of the neutrino transport (Herant et al. 1994; Burrows et al. 1995; Janka \& Müller 1996).

The neutrino-processed outflow becomes proton-rich because the neutron-to-proton mass difference favors positron captures, $\mathrm{e}^{+}+\mathrm{n} \rightarrow \mathrm{p}+\bar{v}_{\mathrm{e}}$, against electron captures, $\mathrm{e}^{-}+\mathrm{p} \rightarrow \mathrm{n}+$ $v_{\mathrm{e}}$, when the electron degeneracy is low, i.e. the electron chemical potential $\mu_{\mathrm{e}} \lesssim k T$ (Beloborodov 2003; Liebendörfer et al. 2003). At large distance from the nascent neutron star, before weak processes freeze out, neutrinos are more energetic and more abundant than electrons and positrons and therefore $v_{\mathrm{e}}$ and $\bar{v}_{\mathrm{e}}$ captures on neutrons and protons, respectively, may be the dominant weak reactions (in particular during the neutrinodriven wind phase). If the $v_{\mathrm{e}}$ and $\bar{v}_{\mathrm{e}}$ spectra are not too dissimilar, the neutron-to-proton mass difference as well as weak magnetism and recoil corrections of the neutrino absorption cross sections (Horowitz \& Li 1999) favor a value of $Y_{\mathrm{e}} \gtrsim 0.5$ (by

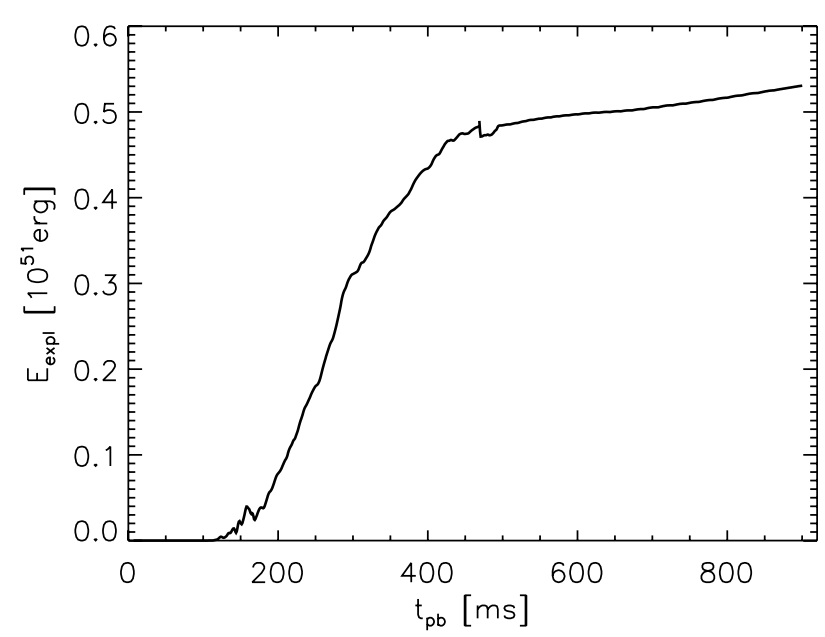

Fig. 38. Explosion energy for Model s15Gio_32.a as defined in Eq. (29). The small jump at $t_{\mathrm{pb}}=468 \mathrm{~ms}$ is a consequence of the mapping of the 2D model to a $1 \mathrm{D}$ grid, and the slow increase afterwards is associated with the power of the neutrino-driven baryonic wind.

reducing the $\bar{v}_{\mathrm{e}}$ absorption opacity relative to the one of $v_{\mathrm{e}}$, see Sect. 3.1.2, Figs. 21, 22 and Appendix A, Fig. A.1) before the weak processes finally freeze out when the expansion timescale becomes shorter than the reaction timescales. The corresponding nucleosynthesis in these neutrino-processed ejecta has been recently investigated in more detail for our Model s15Gio_32.a by Pruet et al. (2005) and independently for 1D explosion models by Fröhlich et al. (2004).

An interesting number is the amount of ${ }^{56} \mathrm{Ni}$ produced by the explosion. Since the simulation was run with a strongly simplified treatment of NSE and nuclear burning, and not with a nuclear reaction network, we can only come up with a crude estimate here. Integrating the mass of all iron-group nuclei at the end of the simulation yields $M_{\text {heavy }}=0.12 M_{\odot}$. This value contains the ${ }^{56} \mathrm{Ni}$ produced by ${ }^{28} \mathrm{Si}$ burning in the shock-heated layers as well as the material ejected from the neutrino-processed region which recombines to iron-group nuclei. Adding the $0.009 M_{\odot}$ of ${ }^{56} \mathrm{Ni}$ produced by oxygen burning 

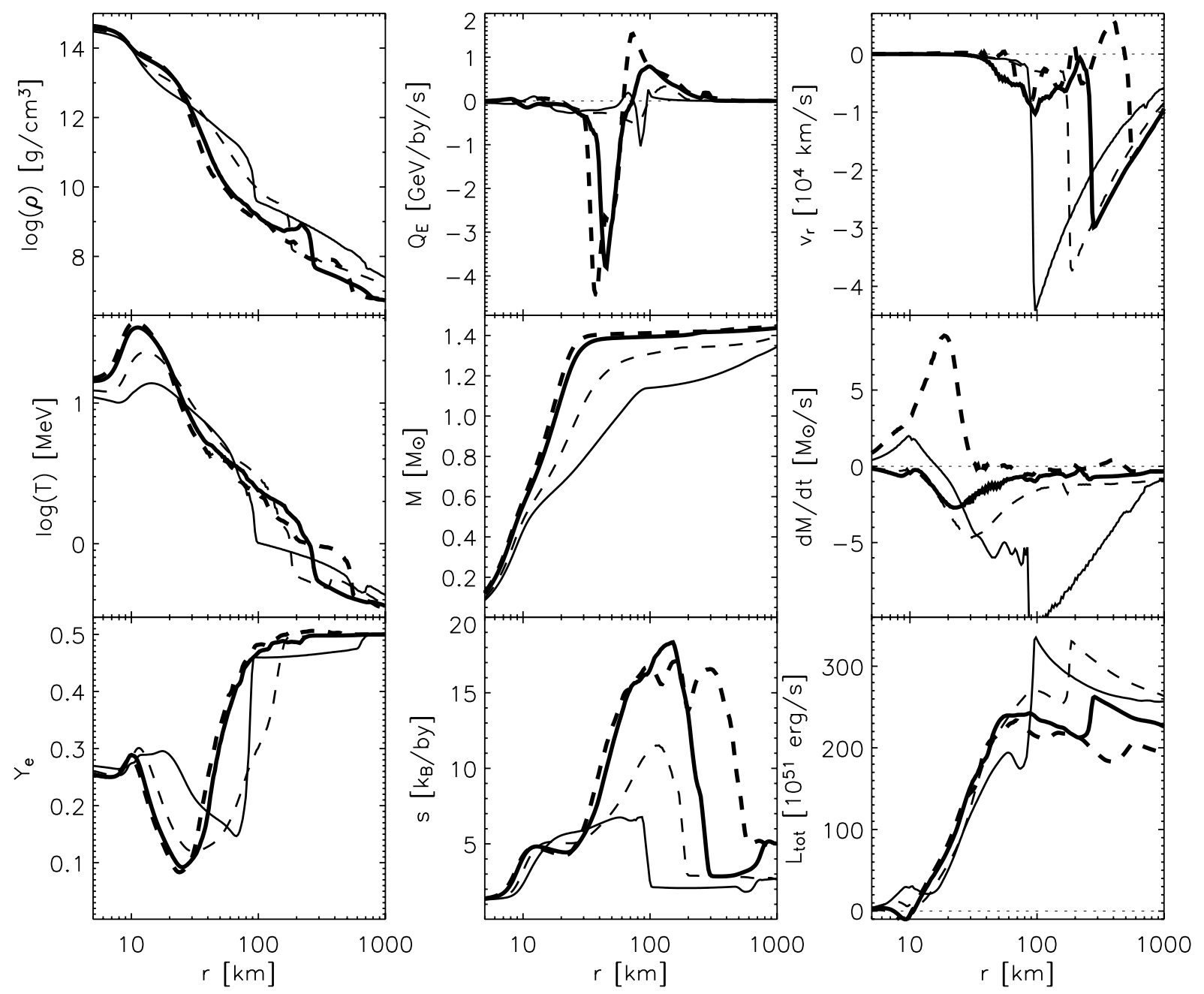

Fig. 39. Radial profiles (laterally averaged) for different quantities at post-bounce times of $11.0 \mathrm{~ms}$ (solid), $72.2 \mathrm{~ms}$ (dashed), $150.1 \mathrm{~ms}$ (thick), and 178.5 ms (thick dashed) for the exploding Model s15Gio_32.a.

in the O-rich Si-layer we obtain a total estimate of $\sim 0.13 M_{\odot}$ of ${ }^{56} \mathrm{Ni}$. Since we assume here that $\mathrm{Si}$ burns to Ni completely and that $\mathrm{Ni}$ is the favored nucleus produced by nucleon recombination in the ejecta (ignoring isotopes and other elements), our number is certainly an upper limit for the true ${ }^{56} \mathrm{Ni}$ yield. Some of this matter is also likely to fall back to the PNS instead of being ejected in our fairly weak explosion, reducing the final $\mathrm{Ni}$ yield even more below our estimate.

Neutrino emission. The evolution of the neutrino luminosities and mean energies is plotted in Fig. 42. We can distinguish four phases: the collapse phase, the prompt burst of electron neutrinos, the accretion phase and, after the onset of the explosion, the beginning of the Kelvin-Helmholtz neutrino cooling phase of the nascent neutron star. The latter is marked by the sudden and pronounced drop of the luminosities which occurs when the accretion of matter onto the PNS is terminated by the start of the explosion. During this early phase of PNS cooling the neutron star deleptonizes, contracts, and heats up. For this reason the mean energies of the emitted neutrinos are still rising and the neutrino luminosities level off to a nearly constant value with no sign of long-time decay. We, however, point out here that our results during this phase depend on the employed approximation for relativistic gravity. The effective potential introduced by Rampp \& Janka (2002) turned out to be a bit too strong and to overestimate the PNS contraction and heating and thus the energies and luminosities of the emitted neutrinos (see Liebendörfer et al. 2005). It also can not ensure the correct Newtonian limit at large radii and thus leads to an overestimation of the gravitational force and fluid acceleration in the infall region ahead of the shock. This may be the reason why the mass accretion rate at late post-bounce times (in nonexploding models) reveals a slight tendency of increase (Fig. 17). The improvement of the treatment of gravity suggested by Marek et al. (2006) should perform better in this respect and might modify the results during the long-time PNS cooling to some degree.

Also note the transient drop of the neutrino luminosity shortly before the $v_{\mathrm{e}}$ burst sets in (around the bounce time), see Fig. 19, upper panel, and Fig. 42, left panel. A detailed analysis of the numerical simulations reveals that this feature correlates with the moment of the most extreme compression of the homologous core and the onset of shock formation. The luminosity minimum is caused by a drop of the density and neutrino emission in the semitransparent regime exterior to the 

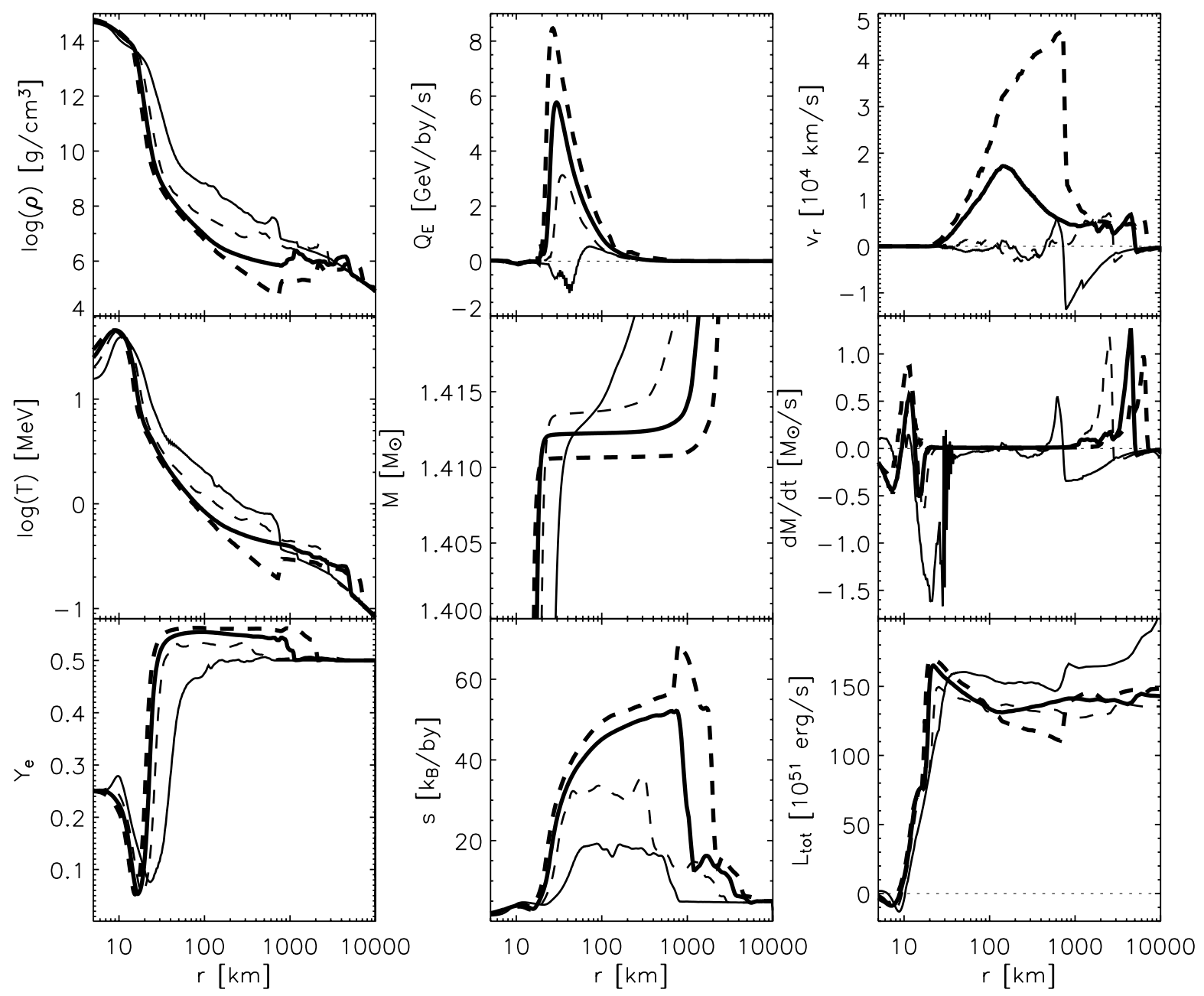

Fig. 40. Radial profiles for different quantities at post-bounce times of $200.2 \mathrm{~ms}$ (solid), $400.6 \mathrm{~ms}$ (dashed), $600.5 \mathrm{~ms}$ (thick solid), and $800.9 \mathrm{~ms}$ (thick dashed) for the exploding Model s15Gio_32.a. For $200.2 \mathrm{~ms}$ and $400.6 \mathrm{~ms}$ laterally averaged information is shown, the two later times correspond to the phase of the evolution after $t=468 \mathrm{~ms}$ which was followed in spherical symmetry.

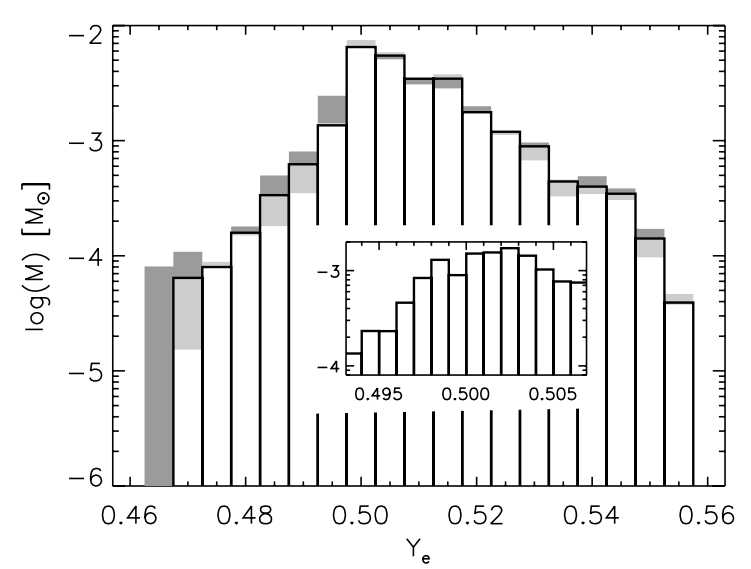

Fig. 41. Mass of neutrino-processed ejecta as a function of the terminal value of the electron fraction $Y_{\mathrm{e}}$ for Model s15Gio_32.a. The masses are calculated with Eq. (30). The solid bars correspond to an evaluation of the outward directed mass flow in the 2D model through a sphere at a radius of $r_{0}=350 \mathrm{~km}$, while the dark and light shading indicate the variation obtained when the analysis is performed at $r_{0}=300 \mathrm{~km}$ and $r_{0}=400 \mathrm{~km}$, respectively. The insert shows the mass distribution for values of $Y_{\mathrm{e}}$ around 0.5 with increased resolution (figure from Pruet et al. 2005). shock formation radius and thus in the non-homologously collapsing layers near the mass shell where the infall velocities reach the maximum value.

The evolution of the neutrino spectra also changes due to the onset of the explosion. Before the explosion sets in, the spectra become gradually harder with time (Fig. 43). This is a consequence of the contraction of the PNS and the persisting inflow and compression-heating of fresh material accreted through the shock. These structural changes lead to an increase of the temperature at the radii at which the neutrinos of given energy decouple from the medium. The increase of this frequency-dependent neutrinospheric temperature is stronger for high-energy neutrinos, leading to an enhanced flux at high energies and thus to a harder spectrum.

While in Model s15Gio_32.b this tendency continues until late times, the spectral evolution changes in Model s15Gio_32.a when the explosion sets in: the mass accretion onto the PNS is abruptly stopped. Now that the inflow of compression-heated matter has ceased, the layer near the PNS surface where the high-energy neutrinos decouple quickly cools and the "neutrinospheric" temperature for high-energy neutrinos does not increase as much as with ongoing accretion 

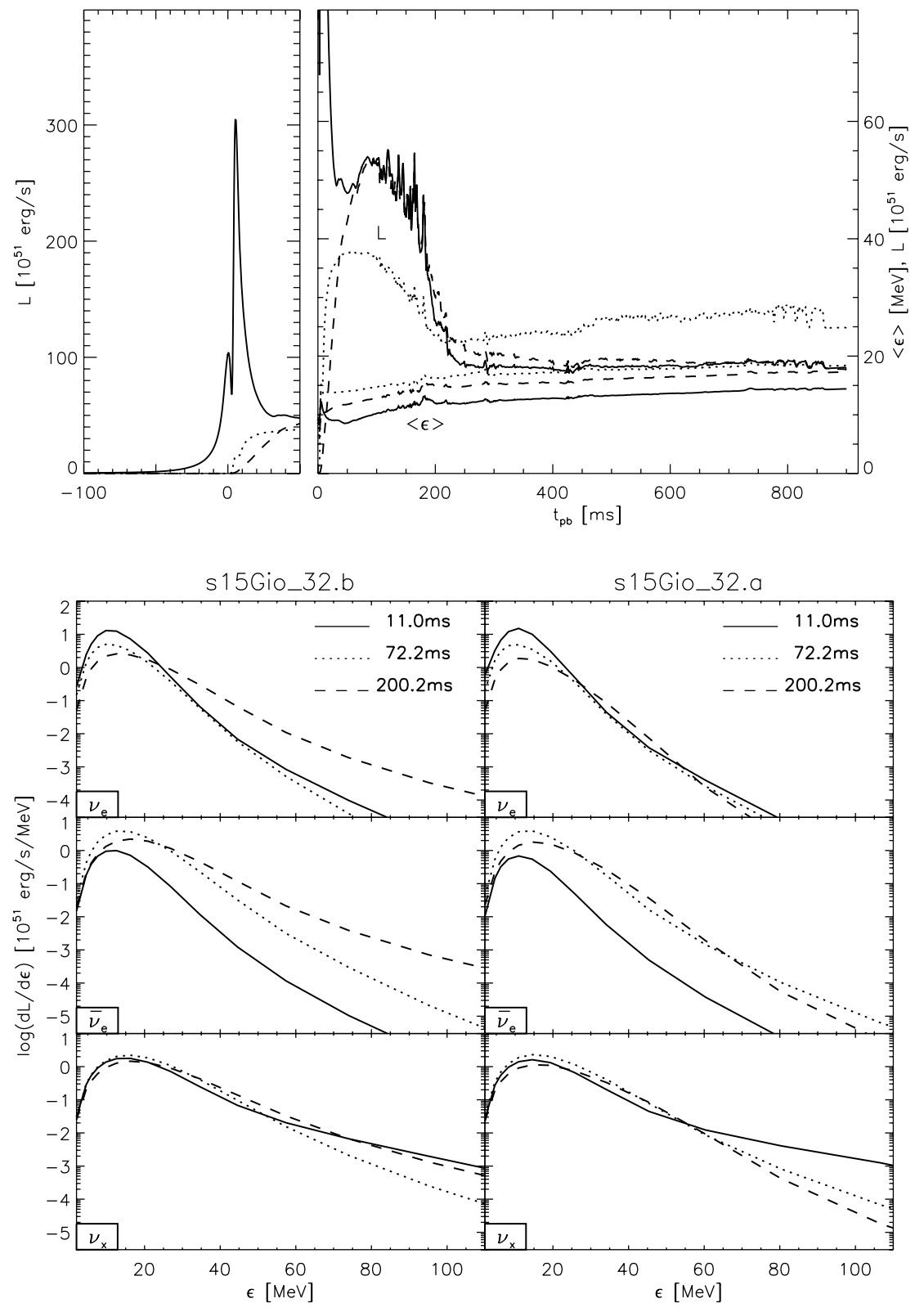

Fig. 42. Evolution of the luminosities for $v_{\mathrm{e}}$ (solid), $\bar{v}_{\mathrm{e}}$ (dashed), and $v_{\mathrm{x}}$ (dotted) in Model s15Gio_32.a around core bounce (left) and during the later post-bounce phases (right), evaluated at a radius of $400 \mathrm{~km}$ for an observer at rest. For the post-bounce phase, we also plot the average energies of the emitted neutrinos.

Fig. 43. Luminosity spectra for $v_{\mathrm{e}}, \bar{v}_{\mathrm{e}}$, and $v_{\mathrm{x}}$ for the 2D Models s15Gio_32.a (right) and s15Gio_32.b (left) for an observer at rest at $400 \mathrm{~km}$. The spectra (given as averages over all directions) are shown for three different post-bounce times: shortly after the peak of the $v_{\mathrm{e}}$ shock breakout burst, when the shock reaches its maximum radius in Model s15Gio_32.b, and shortly after Model s15Gio_32.a has exploded.

(it does not drop either because the energy and lepton number loss lead to contraction). The neutrino spectra no longer harden. This is not in contradiction with the gradual rise of the average neutrino energies which are determined by the spectra around their maxima $(\epsilon \lesssim 20 \mathrm{MeV})$. The details of this differential behaviour in different spectral bands are sensitive to the stellar structure in the neutrino decoupling layer and to the interaction rates and therefore depend also on the neutrino flavor. A deeper analysis goes beyond the scope of this work.

The violent hot bubble convection behind the shock also affects the neutrino emission. When strong downflows hit the cooling region, transient lateral variation of the electron neutrino and antineutrino flux of up to a factor two can occur (Fig. 44a), corresponding to a total luminosity increase in $v_{\mathrm{e}}$ or $\bar{v}_{\mathrm{e}}$ by up to about $20 \%$. Note that our "ray-by-ray" transport scheme tends to overestimate the anisotropy and lateral variability of the neutrino emission (flux and flux spectra, see Fig. 44) produced by the narrowly collimated downflows.
A fully two-dimensional treatment of the transport would certainly reveal more isotropic neutrino emission even when the neutrinos are produced mostly in well localized regions ("hot spots") near the points where the downflows dive into the cooling layer (see e.g. Walder et al. 2005).

Interestingly, sizeable lateral flux variations show up even long after the onset of the explosion, e.g. at $307 \mathrm{~ms}$, caused by downflows which still manage to penetrate inward towards the PNS against the expanding supernova ejecta.

\section{Summary and conclusions}

In this paper (Paper I of a series) we have described the employed equations and numerical implementation of our "ray-by-ray plus" method for multi-group neutrino transport coupled to hydrodynamics in 2D models of stellar core collapse and supernova explosions. The existing code, named MUDBATH, can be extended to a $3 \mathrm{D}$ version in a 


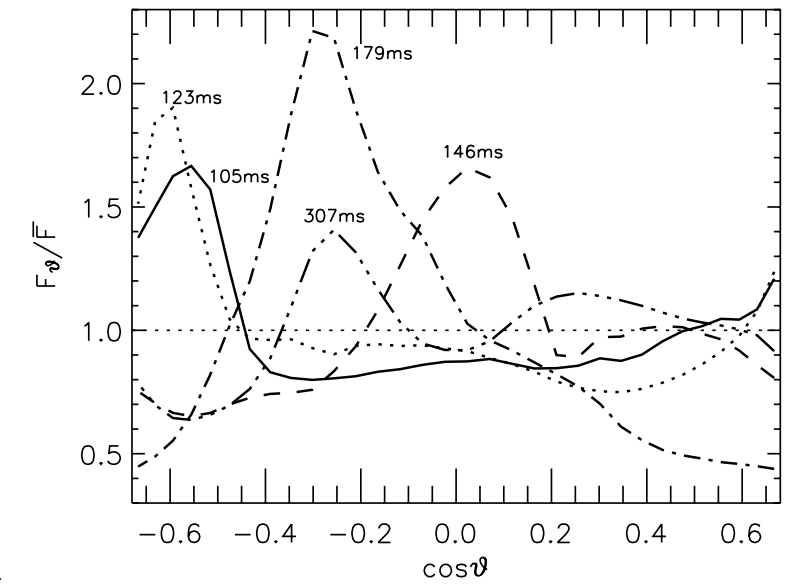

$\mathbf{a}$

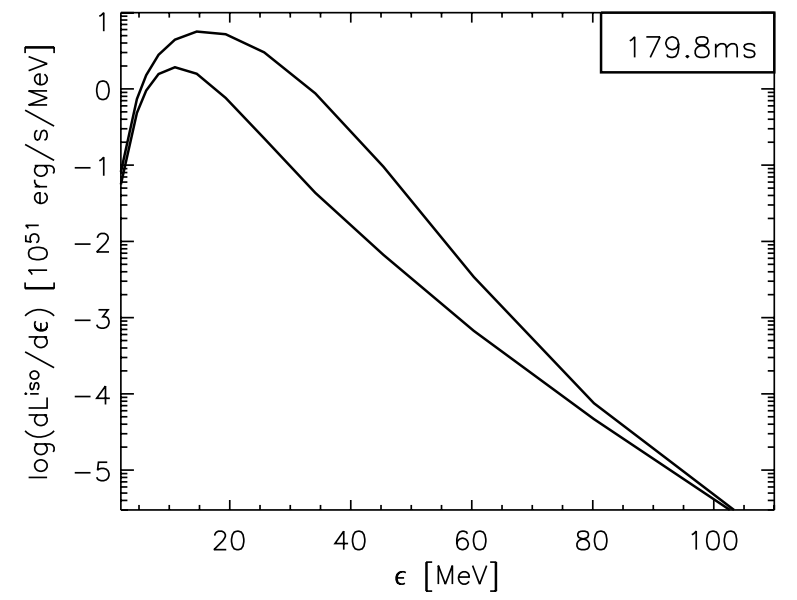

Fig. 44. a) Energy flux $F_{\vartheta}$ of $v_{\mathrm{e}}$ normalized to the average energy flux $\bar{F}$ as function of polar angle $\vartheta$ for different post-bounce times in Model s15Gio_32.a. The results are evaluated for an observer at rest at a radius of $400 \mathrm{~km}$, and the times are picked for showing maximal angular variations. b) For $t=179 \mathrm{~ms}$ after bounce the $v_{\mathrm{e}}$ flux spectra are shown for the lateral directions with maximal and minimal fluxes, again given for an observer at rest at a radius of $400 \mathrm{~km}$. Here, $\mathrm{d} L^{\text {iso }} / \mathrm{d} \epsilon$ is the spectrum corresponding to the "isotropic equivalent luminosity" $L^{\text {iso }}$, calculated by assuming that the flux in one polar direction were representative for all other directions.

straightforward way. Starting from the basic assumption that the neutrino phase space distribution is azimuthally symmetric around the radial direction, which implies that nonradial flux components are zero, we could directly build up the 2D spectral transport scheme from the 1D code version VERTEX of Rampp \& Janka (2002). In course of this, the $O(v / c)$ moments equations for neutrino number, energy, and momentum were extended by the remaining terms containing lateral derivatives, which mediate the coupling between the different angular directions of the polar grid and depend on the lateral component of the fluid velocity. These terms are integrated in an operator splitting step. Closure of the set of moments equations is achieved by using variable Eddington factors, which are computed from the solution of a model Boltzmann equation as in spherical symmetry (cf. Rampp \& Janka 2002). The current code offers the options to do this separately for each angular direction of the grid or - CPU-time saving - once on a laterally averaged stellar background.

In addition to the velocity-dependent terms with lateral derivatives in the transport part of the code, we also take into account the lateral components of neutrino pressure gradients in the hydrodynamics equations at optically thick conditions.

We provided a compilation of tests for our numerical scheme and input physics, by which we attempted to assess potential limits of its applicability or accuracy in the context of core collapse calculations. In particular the following points were addressed:

(1) Our approximation of general relativity (i.e., replacing Newtonian gravity by an effective relativistic gravitational potential and including redshift and time dilation in the neutrino transport; cf. Rampp \& Janka 2002) was tested against fully relativistic calculations in spherical symmetry (Liebendörfer et al. 2005; Marek et al. 2006). Very good agreement in all relevant quantities was found for cases with moderately relativistic fluid velocities (up to $\sim 10-20 \%$ of the speed of light) at least until several $100 \mathrm{~ms}$ after bounce. Moreover, by 2D simulations with different polar wedges and lateral boundary conditions we demonstrated empirically that linear momentum conservation, which cannot be strictly guaranteed with the use of our effective relativistic potential in multi-dimensional simulations (see Marek et al. 2006), is satisfactorily well fulfilled in practical applications.

(2) We explored the role of the lateral derivatives in the moments equations and of the lateral components of neutrino pressure gradients in the hydrodynamics equations. Both turned out to be necessary extensions when nonequilibirium transport is applied to the multidimensional case and artificial fluid acceleration and buoyancy shall be avoided. The latter may occur in situations where neutrinos contribute significantly to the gas pressure and are strongly coupled to stellar medium so that their advection with moving fluid elements cannot be ignored. Our treatment of neutrino-hydrodynamics in two dimensions produces results in agreement with the expectations from a stability analysis of the stellar fluid, which we performed with a "Quasi-Ledoux" criterion that tries to take into account (in a simple way) the lepton number exchange by neutrino diffusion between moving fluid elements and their surroundings. This at least shows that our scheme does not artificially instigate convective activity, although, of course, we cannot exclude that disregarding lateral components of the neutrino number and energy fluxes in our code underestimates neutrino diffusion and thus hampers or suppresses the development of doubly diffusive instabilities below the neutrinosphere (in particular since their occurrence seems to be very sensitive to the efficiency of diffusion; cf. Bruenn et al. 2004).

(3) Our transport treatment, favoring radial streaming, potentially overestimates the magnitude of asymmetry and angular contrast of the radiation field of neutrinos that stream off from the neutrinosphere or cooling layer. When downflows or convective eddies create hot spots with enhanced 
neutrino emission, material at the involved latitudes exterior to these emission regions receives more radiation, whereas the exposure of neighboring lateral directions is underestimated. Our hydrodynamical simulations revealed that the corresponding angular anisotropy is nonstationary and varies quickly in space and time on timescales of several milliseconds. Short, transient, local flux enhancements reach typically some $10 \%$ up to occasionally a factor of two compared to the average value. Performing a postprocessing test by making the extreme assumption that the neutrino radiation field is spherically symmetric (with the same value of the total luminosity) we found that the total heating rate in the gain layer is not significantly changed at any time. The average heating rate is changed only by a few percent and the heating rate per baryon even less. Distinguishing in the postprocessing evaluation between downflows and rising hot bubbles, we determined a decrease or increase, respectively, of roughly $10 \%$ in the timeaveraged net heating. We therefore conclude that underestimating the spherical symmetry of the neutrino emission is not very likely to produce dynamical consequences for the supernova evolution in our simulations.

(4) We performed a number of $1 \mathrm{D}$ tests to assess the consequences of an error in the EoS of Lattimer \& Swesty, which is usually used in our core collapse simulations at NSE conditions and at densities above $6 \times 10^{7} \mathrm{~g} \mathrm{~cm}^{-3}$. This error leads to an underestimation of the mass fraction of $\alpha$ particles and corresponding effects in the other thermodynamical quantities like pressure, entropy, and temperature for given density, internal energy density, and electron fraction. Replacing the Lattimer \& Swesty EoS below a density of $10^{11} \mathrm{~g} \mathrm{~cm}^{-3}$ by different versions of a low-density EoS with 4 or 17 nuclear species (neutrons, protons, $\alpha$ 's and one or 14 heavy nuclei, respectively) in NSE reveals the expected differences of the dependent thermodynamical variables behind the shock, but negligibly small effects in the post-bounce evolution of the models, e.g., nearly identical shock trajectories. We determined two reasons for this insensitivity: firstly, the postshock entropies in our simulations with relativistic gravity are so high that $\alpha$ particles play a relatively unimportant role and therefore the EoS differences are rather small (of order 10\%). Secondly, the pressure profile in the essentially hydrostatic layer behind the standing accretion shock is tightly constrained and thus determined by the gravitational field of the nascent neutron star on the one hand, and by the mass accretion rate and jump conditions at the shock on the other hand. Differences of density and temperature in the gain layer, of course, affect the neutrino heating, which, however, is dynamically not very relevant in our models which are "quite far" from an explosion.

(5) Finally, we investigated the implications of wiggles in the entropy profile (of about $10 \%$ amplitude), which we discovered in the deceleration layer behind a standing accretion shock, when a physical EoS is employed in which the energy density includes contributions from the particle rest masses. This numerical phenomenon seems to be linked to the use of a Riemann solver in our hydrodynamics code and turned out to be sensitive to the ratio of pressure to energy density fed into the Riemann solver. The artificial entropy wiggles disappeared after we applied an algorithmic procedure which allows us to extract the rest-mass contributions from the energy density of the EoS so that the true internal energy is evolved in the hydrodynamics part of the code. Comparative calculations revealed that the ugly entropy fluctuations did not lead to any significant dynamical effects in 1D as well as 2D supernova simulations.

In the present paper our new neutrino-hydrodynamics code was applied to 1D and 2D simulations of stellar core collapse and post-bounce evolution of a $15 M_{\odot}$ progenitor star. Our aim was to investigate the effects of different input physics. We also explored modifications in the neutrino transport, which change the neutrino-matter coupling in the heating and cooling layers outside of the neutrinosphere. Our findings can be summarized as follows:

(a) None of our simulations with the most complete implementation of neutrino transport yields an explosion, neither in spherical symmetry nor in two dimensions. The $2 \mathrm{D}$ models were computed with a lateral wedge of about 90 degrees around the equatorial plane and periodic boundary conditions, a setup which is similar to the one used in the first generation of models of convectively supported, neutrinodriven supernova explosions (Herant et al. 1994; Burrows et al. 1995; Fryer 1999). Our models with the accurate spectral description of neutrino transport and neutrino-matter interactions are therefore unable to reproduce the success of these previous simulations, in which a simplified treatment of neutrinos by grey, flux-limited diffusion was applied. Since the transport is the major difference between the older models and our current ones (of course, there are other differences, too, numerical as well as in the input physics), we interpret our negative results as a confirmation of concerns raised by Mezzacappa et al. (1998b), who suspected that the transport simplifications might have favored the explosions found by Herant et al. (1994) and Burrows et al. (1995).

(b) Using a $90^{\circ}$ wedge with periodic boundary conditions, however, imposes constraints on the size of the nonradial structures (i.e., the wavelengths of the modes) which are allowed to exist. It also prevents one from studying the growth of low (dipole, $l=1$, and quadrupole, $l=2$ ) modes in the postshock flow as seen in neutrinoless (adiabatic) simulations of Blondin et al. (2003) and predicted as a consequence of the vortical-acoustic cycle (Foglizzo 2002). In Paper II we will demonstrate by 2D simulations with a full 180 degree grid that a thus less constrained flow can indeed develop a dominant low-mode pattern behind a highly deformed shock front, which performs bipolar oscillations. This can lead not only to quantitative but even qualitative differences in the post-bounce evolution of the collapsing stellar core, deciding about explosion or non-explosion.

(c) The sensitivity to the treatment of the neutrino transport became evident once more from two simulations which we performed without the terms in the neutrino momentum 
equation, which depend on the radial velocity (the terms responsible for neutrino advection were, however, included). In contrast to the corresponding simulations with the most complete transport implementation these two models produced explosions, caused by roughly a factor of two less energy loss and more neutrino heating due to $20-30 \%$ higher neutrino energy densities in the cooling and heating layers, respectively. These layers between neutrinosphere and stalled shock in the 1D model underwent a number of nonlinear, radial oscillations with growing amplitude and final runaway, similar to the $\kappa$ mechanism of pulsational instability of stellar atmospheres. The 2D model developed very strong postshock convection and started to explode when the interface between silicon shell and oxygen-rich silicon shell at $1.42 M_{\odot}$ reached the shock about $150 \mathrm{~ms}$ after core bounce. The net explosion energy was rather low, roughly $0.5 \times 10^{51} \mathrm{erg}$. The explosion left behind a nascent neutron with an initial baryonic mass of about $1.41 M_{\odot}$ and produced less than $\sim 0.13 M_{\odot}$ of iron-group elements. The latter two numbers may change by the effects of the subsequent neutrino-driven baryonic wind and later fallback. Most interesting of this "artificial" explosion, however, is the electron fraction $Y_{\mathrm{e}}$ in the neutrinoheated ejecta. Most of this material has values above 0.5 and less than about $10^{-4} M_{\odot}$ have $Y_{\mathrm{e}} \lesssim 0.47$, in agreement with constraints derived from observed galactic chemical abundances (Hoffman et al. 1996). The model therefore does not show the enormous overproduction of $N=50$ closed neutron shell nuclei found in previous simulations (see also Pruet et al. 2005). Values of the electron fraction above 0.5 are a consequence of our accurate, spectral description of electron neutrino and antineutrino transport, enhanced by the inclusion of weak magnetism corrections in the charged-current reactions with nucleons. These corrections reduce the absorption of $\bar{v}_{\mathrm{e}}$ relative to that of $v_{\mathrm{e}}$ in the neutrino-heated ejecta (cf. Horowitz \& Li 1999). The proton richness should not depend on the manipulation of the neutrino transport which caused the explosion, because electron neutrinos and antineutrinos were affected in the same way. Moreover, values of $Y_{\mathrm{e}}$ above 0.5 were also obtained in the early phase of the neutrinodriven wind, which set in (at $\sim 300 \mathrm{~ms}$ p.b.) after the supernova explosion had been lauched and which we followed from $\sim 470 \mathrm{~ms}$ to more than a second after bounce in 1D with the velocity-dependent terms in the neutrino momentum equation turned on again. Finally, our result is supported by Fröhlich et al. (2004), who used spherically symmetric general relativistic simulations in which the numerical treatment of the transport differed from ours and in which explosions were achieved by other modifications of the transport than in our work.

(d) Our 1D models reveal the existence of a (Quasi-)Ledouxunstable layer below the neutrinosphere. In the corresponding 2D simulations convective activity inside the nascent neutron star sets in about $30 \mathrm{~ms}$ after bounce and persists until the end of the simulated evolution. Since the convective layer nearly reaches with the muon and tau neutrinosphere, the luminosity of these neutrinos is enhanced.
A detailed analysis of the consequences of this convective activity for neutrino emission and supernova evolution will be given in Paper II.

(e) Improving neutrino-nucleon interactions by the effects of nucleon thermal motions and recoil and weak-magnetism corrections, and including pair annihilation and scattering between neutrinos of different flavors and nucleon-nucleon bremsstrahlung, we found important differences in many aspects of the transport (in spectra as well as luminosities) for neutrinos of all flavors. This leads to quantitative differences of the post-bounce dynamics of the collapsing stellar core and to differences in the shock evolution, without, however, causing a qualitative change of the outcome of the simulations.

In Paper II we shall present simulations for different progenitors between $11 M_{\odot}$ and $25 M_{\odot}$, among which we will also consider a case with rotation. We will explore the role of pre-collapse random perturbations in the core, will study the effects of convection below the neutrinosphere, and will investigate the implications of hydrodynamic instabilities - convective and vortical-acoustic - in the layer between neutrinosphere and stalled supernova shock by varying the angular resolution and the size of the lateral wedge of the polar grid. Some results of these models were already published in Buras et al. (2003b) and Janka et al. (2004, 2005b,a).

The spectral treatment of neutrino transport and neutrinomatter interactions applied in these models means a new level of refinement and accuracy in two-dimensional hydrodynamical supernova simulations. But our transport scheme has advantages as well as disadvantages. On the positive side is the fact that it was developed on grounds of a well tested code for spherically symmetric problems and thus guarantees to produce results of well-known good quality in an important limit. Moreover, our neutrino-hydrodynamics code possesses good coarse level parallelism, which allows the efficient use of a large number of processors. Due to the fully implicit time-differencing and the corresponding matrix inversions in the transport part, however, systems with a low-latency, highbandwidth memory are preferable. As we argued in this paper and presented tests for, we believe that our transport treatment is a reasonably good approach for problems in which local macroscopic anisotropies occur but which still have an overall spherical geometry. The applicability of our method, however, becomes questionable in situations with large, global deformation, because in this case our basic assumption of (on average) azimuthal symmetry of the neutrino intensity is certainly inadequate. On the negative side there are also substantial CPU-time requirements. The disregard of neutrino flux components in the angular directions tends to cause an overestimation of the angular asymmetry of neutrinos streaming out from the nascent neutron star. This may be a handicap when accurate information about the corresponding directional variation is required, e.g., for estimating pulsar kicks or gravitational wave signals associated with the anisotropic neutrino emission. Future tests will also have to clarify the question whether the disregard of nonradial neutrino flux components leads to a suppression of doubly diffusive instabilities inside the neutron star. 
A comparison with other transport methods, which might employ alternative, complementary approximations to deal with the high dimensionality of transport in multi-dimensional problems, is therefore highly desirable and in fact indispensable for verification and validation of our approach. We are therefore looking forward to compare our results with those obtained by other groups.

Acknowledgements. We are grateful to Matthias Keil, Georg Raffelt, and Leonhard Scheck for helpful discussions, to Kohji Takahashi for adapting the neutrino rates of Burrows \& Sawyer $(1998,1999)$ for use in our code, and to Charles Horowitz for providing us with the weak magnetism corrections in neutral and charged-current neutrinonucleon interactions. We also thank Christian Cardall for pointing out missing terms in the hydrodynamics equations written in Eqs. (2)-(4). R.B. would especially like to thank Francisco Kitaura for helping to solve the problem of the entropy wiggles. We thank the Institute for Nuclear Theory of the University of Seattle for its hospitality during an early stage of this work. Support by the Sonderforschungsbereich 375 on "Astro-Particle Physics" of the Deutsche Forschungsgemeinschaft is acknowledged. The computations were performed on the NEC SX-5/3C and the IBM p690 "Regatta" system of the Rechenzentrum Garching, and on the Cray T90 of the John von Neumann Institute for Computing (NIC) in Jülich.

\section{References}

Beloborodov, A. M. 2003, ApJ, 588, 931

Bethe, H. A. 1990, Rev. Mod. Phys., 62, 801

Bethe, H. A., Brown, G. E., \& Cooperstein, J. 1987, ApJ, 322, 201

Blondin, J. M., Mezzacappa, A., \& DeMarino, C. 2003, ApJ, 584, 971

Bruenn, S. W. 1985, ApJS, 58, 771

Bruenn, S. W., \& Dineva, T. 1996, ApJ, 458, L71

Bruenn, S. W., \& Mezzacappa, A. 1994, ApJ, 433, L45

Bruenn, S. W., \& Mezzacappa, A. 1997, Phys. Rev. D, 56, 7529

Bruenn, S. W., Mezzacappa, A., \& Dineva, T. 1995, Phys. Rep., 256, 69

Bruenn, S. W., Raley, E. A., \& Mezzacappa, A. 2004 [arXiv: astro-ph/0404099]

Buras, R., Janka, H.-T., Keil, M.-T., Raffelt, G., \& Rampp, M. 2003a, ApJ, 587, 320

Buras, R., Rampp, M., Janka, H.-T., \& Kifonidis, K. 2003b, Phys. Rev. Lett., 90, 241101

Buras, R., Janka, H.-T., Rampp, M., \& Kifonidis, K. 2005, in preparation (Paper II)

Burrows, A. 1987, ApJ, 318, L57

Burrows, A., \& Fryxell, B. A. 1992, Science, 258, 430

Burrows, A., \& Fryxell, B. A. 1993, ApJ, 418, L33

Burrows, A., Hayes, J., \& Fryxell, B. A. 1995, ApJ, 450, 830

Burrows, A., \& Lattimer, J. 1988, Phys. Rep., 163, 51

Burrows, A., \& Sawyer, R. F. 1998, Phys. Rev. C, 58, 554

Burrows, A., \& Sawyer, R. F. 1999, Phys. Rev. C, 59, 510

Cardall, C., \& Mezzacappa, A. 2003, Phys. Rev. D, 68, 023006

Cardall, C. Y., Lentz, E. J., \& Mezzacappa, A. 2005, Phys. Rev. D, 72, 043007

Carter, G. W., \& Prakash, M. 2002, Phys. Lett. B, 525, 249

Cernohorsky, J. 1994, ApJ, 433, 247

Colella, P., \& Woodward, P. 1984, J. Comp. Phys., 54, 174

Einfeldt, B. 1988, SIAM Jour. Numer. Anal., 25, 294

Epstein, R. I. 1979, MNRAS, 188, 305
Foglizzo, T. 2001, A\&A, 368, 311

Foglizzo, T. 2002, A\&A, 392, 353

Foglizzo, T., \& Tagger, M. 2000, A\&A, 363, 174

Fröhlich, C., Hauser, P., Liebendörfer, M., et al. 2004, ApJ, in press [arXiv: astro-ph/0410208]

Fryer, C. L. 1999, ApJ, 522, 413

Fryer, C. L., \& Heger, A. 2000, ApJ, 541, 1033

Fryer, C. L., \& Warren, M. S. 2002, ApJ, 574, L65

Fryer, C. L., \& Warren, M. S. 2004, ApJ, 601, 391

Fryxell, B., Müller, E., \& Arnett, W. 1989, Hydrodynamics and Nuclear Burning, preprint MPA-449 (Garching: Max Planck Institut für Astrophysik)

Fryxell, B., Olson, K., Ricker, P., et al. 2000, ApJS, 131, 273

Fuller, G. M., \& Qian, Y.-Z. 2005 [arXiv: astro-ph/0505240]

Hamuy, M. 2003, ApJ, 582, 905

Hannestad, S., Janka, H.-T., Sigl, G., \& Raffelt, G. 2000, Phys. Rev. D, 62, 093021

Hannestad, S., \& Raffelt, G. 1998, ApJ, 507, 339

Herant, M., Benz, W., \& Colgate, S. 1992, ApJ, 395, 642

Herant, M., Benz, W., Hix, W. R., Fryer, C. L., \& Colgate, S. A. 1994, ApJ, 435, 339

Hoffman, R. D., Woosley, S. E., Fuller, G. M., \& Meyer, B. S. 1996, ApJ, 460, 478

Horowitz, C. 1997, Phys. Rev. D, 55, 4577

Horowitz, C. J. 2002, Phys. Rev. D, 65, 043001

Horowitz, C. J., \& Li, G. 1999, Phys. Rev. Lett., 82, 5198

Janka, H.-T. 1991, Ph.D. Thesis, Technische Universität München, preprint MPA-587

Janka, H.-T. 1999, unpublished

Janka, H.-T. 2001, A\&A, 368, 527

Janka, H.-T. 2004, in Young Neutron Stars and Their Environments, IAU Symp. 218, Sydney, Australia, July 14-17, 2003, ed. F. Camilo, \& B. Gaensler, (San Francisco: Astronomical Society of the Pacific), ASP Conf. Proc., 3

Janka, H.-T., \& Keil, W. 1998, in Supernovae and Cosmology, ed. L. Labhardt, B. Binggeli, \& R. Buser (Astronomisches Institut, Universität Basel), 7

Janka, H.-T., \& Müller, E. 1993, in Proc. of the Int. Symposium on Neutrino Astrophysics, Takayama/Kamioka, Japan, October 19-22, 1992, ed. Y. Suzuki, \& K. Nakamura (Tokio: Universal Academy Press), 203

Janka, H.-T., \& Müller, E. 1994, A\&A, 290, 496

Janka, H.-T., \& Müller, E. 1995, ApJ, 448, L109

Janka, H.-T., \& Müller, E. 1996, A\&A, 306, 167

Janka, H.-T., Buras, R., Kitaura, F. S., et al. 2004 [arXiv: astro-ph/0411347]

Janka, H.-T., Buras, R., Kifonidis, K., Marek, A., \& Rampp, M. 2005a, in Springer Proceedings in Physics, 99, Cosmic Explosions, On the 10th Anniversary of SN1993J. ed. J. Marcaide, \& K. Weiler (Berlin: Springer), Proc. IAU Coll., 192, 253

Janka, H.-T., Buras, R., Kitaura, F. S., Marek, A., \& Rampp, M. 2005b, in Proc. of 12th Workshop on Nuclear Astrophysics, Ringberg Castle, Tegernsee, Germany, March 22-27, 2004, ed. E. Müller, \& H.-T. Janka (Garching: MPI für Astrophysik), 150

Janka, H.-T., Scheck, L., Kifonidis, K., Müller, E., \& Plewa, T. 2005c, in The Fate of the Most Massive Stars, ASP Conf. Ser., 332, Proc. of the conference held 23-28 May, 2004 in Grand Teton National Park, Wyoming, ed. R. Humphreys, \& K. Stanek (San Fransisco: Astronomical Society of the Pacific), 372

Kaneko, N., Morita, K., \& Maekawa, M. 1984, Ap\&SS, 107, 333

Keil, M.-T., Raffelt, G., \& Janka, H.-T. 2003, ApJ, 590, 971

Keil, W. 1997, Ph.D. Thesis, Technische Universität München Keil, W., \& Janka, H.-T. 1995, A\&A, 296, 145 
Keil, W., Janka, H.-T., \& Müller, E. 1996, ApJ, 473, L111

Kifonidis, K., Plewa, T., Janka, H.-T., \& Müller, E. 2003, A\&A, 408, 621

Kippenhahn, R., \& Weigert, A. 1990, Stellar Structure and Evolution, Astronomy and Astrophysics Library (Berlin: Springer)

Langanke, K., Martínez-Pinedo, G., \& Sampaio, J. M. 2001, Phys. Rev. C, 64, 055801

Langanke, K., Martínez-Pinedo, G., Sampaio, J. M., et al. 2003, Phys. Rev. Lett., 90, 241102

Lattimer, J., \& Swesty, F. 1991, Nucl. Phys. A, 535, 331

Liebendörfer, M., Mezzacappa, A., Thielemann, F., et al. 2001, Phys. Rev. D, 63, 3004

Liebendörfer, M., Mezzacappa, A., Messer, O. E. B., et al. 2003, Nucl. Phys. A, 719, C144

Liebendörfer, M., Rampp, M., Janka, H.-T., \& Mezzacappa, A. 2005, ApJ, 620, 840

Liou, M. 2000, J. Comp. Phys., 160, 623

Livne, E., Burrows, A., Walder, R., Lichtenstadt, I., \& Thompson, T. A. 2004, ApJ, 609, 277

Marek, A., Dimmelmeier, H., Janka, H.-T., Müller, E., \& Buras, R. 2006, A\&A, 445, 273

Mezzacappa, A., \& Blondin, J. M. 2003, in From Twilight to Highlight: The Physics of Supernovae, ed. W. Hillebrandt, \& B. Leibundgut (Berlin: Springer), 63

Mezzacappa, A., \& Bruenn, S. W. 1993a, ApJ, 405, 669

Mezzacappa, A., \& Bruenn, S. W. 1993b, ApJ, 410, 740

Mezzacappa, A., \& Bruenn, S. W. 1993c, ApJ, 405, 637

Mezzacappa, A., Calder, A. C., Bruenn, S. W., et al. 1998a, ApJ, 493, 848

Mezzacappa, A., Calder, A. C., Bruenn, S. W., et al. 1998b, ApJ, 495, 911

Mihalas, D., \& Mihalas, B. 1984, Foundations of Radiation Hydrodynamics (New York: Oxford University Press)

Miralles, J. A., Pons, J. A., \& Urpin, V. A. 2000, ApJ, 543, 1001

Müller, E. 1993, in Proc. of 7th Workshop on Nuclear Astrophysics, Ringberg Castle, Tegernsee, Germany, March 22-27, 1993, Report MPA/P7, ed. W. Hillebrandt, \& E. Müller (Garching: MPI für Astrophysik), 27

Müller, E., \& Steinmetz, M. 1995, Comp. Phys. Comm., 89, 45

Plewa, T., \& Müller, E. 1999, A\&A, 342, 179

Pons, J. A., Miralles, J. A., \& Ibanez, J. M. A. 1998, A\&AS, 129, 343
Pons, J. A., Reddy, S., Prakash, M., Lattimer, J. M., \& Miralles, J. A. 1999, ApJ, 513, 780

Pruet, J., Woosley, S. E., Buras, R., Janka, H.-T., \& Hoffman, R. D. 2005, ApJ, 623, 325

Quirk, J. J. 1994, Int. J. Num. Meth. Fluids, 18, 555

Raffelt, G. G. 2001, ApJ, 561, 890

Rampp, M. 2000, Ph.D. Thesis, Technische Universität München

Rampp, M., \& Janka, H.-T. 2002, A\&A, 396, 361

Ratković, S., Dutta, S. I., \& Prakash, M. 2003, Phys. Rev. D, 67, 123002

Reddy, S., Prakash, M., \& Lattimer, J. M. 1998, Phys. Rev. D, 58, 3009

Reddy, S., Prakash, M., Lattimer, J. M., \& Pons, J. 1999, Phys. Rev. C, 59,2888

Scheck, L. 2005, Ph.D. Thesis, Technische Universität München, in preparation

Scheck, L., Plewa, T., Janka, H.-T., Kifonidis, K., \& Müller, E. 2004, Phys. Rev. Lett., 92, 011103

Schinder, P. J. 1990, ApJS, 74, 249

Sutherland, R. S., Bisset, D. K., \& Bicknell, G. V. 2003, ApJS, 147, 187

Swesty, F. D., \& Myra, E. S. 2005, proc. of "Open Issues in CoreCollapse Supernovae", in press [arXiv: astro-ph/0506178]

Thielemann, F.-K., Argast, D., Brachwitz, F., et al. 2003, Nucl. Phys. A, 718, 139

Thompson, T. A., Burrows, A., \& Horvath, J. E. 2000, Phys. Rev. C, 62, 035802

Thompson, T. A., Burrows, A., \& Pinto, P. A. 2003, ApJ, 592, 434

Thompson, T. A., Quataert, E., \& Burrows, A. 2005, ApJ, 620, 861

Tomàs, R., Kachelrieß, M., Raffelt, G., et al. 2004, J. Cosmol. and Astro-Particle Phys., 9, 15

Walder, R., Burrows, A., Ott, C. D., et al. 2005, ApJ, 626, 317

Wilson, J. R., \& Mayle, R. 1988, Phys. Rep., 163, 63

Wilson, J. R., \& Mayle, R. 1993, Phys. Rep., 227, 97

Wilson, J. R., Mayle, R., Woosley, S., \& Weaver, T. 1986, in Annals of the New York Academy of Sciences, 470, Twelfth Texas Symposium on Relativistic Astrophysics, ed. M. Livio, \& G. Shaviv, 267

Woosley, S. E., Heger, A., \& Weaver, T. A. 2002, Rev. Mod. Phys., 74,1015

Woosley, S. E., \& Weaver, T. A. 1995, ApJS, 101, 181 
R. Buras et al.: Two-dimensional hydrodynamic core-collapse supernova simulations with spectral neutrino transport. I., Online Material p 1

\section{Online Material}




\section{Appendix A: Neutrino opacities}

Table A.1. Overview of all neutrino-matter interactions currently implemented in our Supernova code as our set of "improved opacities" (in contrast to the set of opacities listed in Rampp \& Janka (2002), which we call our "standard case" containing a number of approximations removed in the current improved treatment). For each type of interaction we refer to the relevant section in this work or point to references where the fundamental aspects of the calculation of the corresponding rate are summarized and details of its numerical implementation are given. The third column lists references where comprehensive information can be found about the physics and the approximations employed in the rate calculations. In the first column the symbol $v$ represents any of the neutrinos $v_{\mathrm{e}}, \bar{v}_{\mathrm{e}}, v_{\mu}, \bar{v}_{\mu}, v_{\tau}, \bar{v}_{\tau}$, the symbols $\mathrm{e}^{-}, \mathrm{e}^{+}, \mathrm{n}, \mathrm{p}$, and $A$ denote electrons, positrons, free neutrons and protons, and heavy nuclei, respectively. The symbol $\mathrm{N}$ means $\mathrm{n}$ or $\mathrm{p}$.

\begin{tabular}{|c|c|c|c|c|}
\hline Reaction & & & Implementation described in & References \\
\hline$v \mathrm{e}^{ \pm}$ & $\rightleftharpoons$ & $v \mathrm{e}^{ \pm}$ & Rampp \& Janka (2002) & Mezzacappa \& Bruenn (1993b); Cernohorsky (1994) \\
\hline$v A$ & $\rightleftharpoons$ & $v A$ & Rampp \& Janka (2002) & Horowitz (1997); Bruenn \& Mezzacappa (1997) \\
\hline$v \mathrm{~N}$ & $\rightleftharpoons$ & $v \mathrm{~N}$ & Sect. A.1 & Burrows \& Sawyer (1998) \\
\hline$v_{\mathrm{e}} \mathrm{n}$ & $\rightleftharpoons$ & $e^{-} p$ & Sect. A.1 & Burrows \& Sawyer (1999) \\
\hline $\bar{v}_{\mathrm{e}} \mathrm{p}$ & $\rightleftharpoons$ & $e^{+} n$ & Sect. A.1 & Burrows \& Sawyer (1999) \\
\hline$v_{\mathrm{e}} A^{\prime}$ & $\rightleftharpoons$ & $\mathrm{e}^{-} A$ & Rampp \& Janka (2002) & Bruenn (1985); Mezzacappa \& Bruenn (1993c) \\
\hline$v \bar{v}$ & $\rightleftharpoons$ & $e^{-} e^{+}$ & Rampp \& Janka (2002) & Bruenn (1985); Pons et al. (1998) \\
\hline$\nu \bar{v} \mathrm{NN}$ & $\rightleftharpoons$ & $\mathrm{NN}$ & Rampp \& Janka (2002) & Hannestad \& Raffelt (1998) \\
\hline$v_{\mu, \tau} \bar{v}_{\mu, \tau}$ & $\rightleftharpoons$ & $v_{\mathrm{e}} \bar{v}_{\mathrm{e}}$ & Buras et al. (2003a) & see Buras et al. (2003a) \\
\hline$\stackrel{(-)}{v}_{\mu, \tau} \stackrel{(-)}{v_{\mathrm{e}}}$ & $\rightleftharpoons$ & $\stackrel{(-)}{v}_{\mu, \tau}{\stackrel{(-)}{v_{\mathrm{e}}}}$ & Buras et al. (2003a) & see Buras et al. (2003a) \\
\hline
\end{tabular}

The description of the neutrino opacities which we call our "standard" set closely follows Bruenn (1985) and Mezzacappa \& Bruenn (1993a,b) with the only exception that we, in addition, take into account neutrino pair processes due to nucleon-nucleon bremsstrahlung. A complete list of the considered reactions, corresponding pointers to the literature and details of the numerical implementation into our transport code can be found in the appendix of Rampp \& Janka (2002).

The "improved description" of neutrino opacities which is used in our latest simulations comprises the reactions summarized in Table A.1. Specifically, the so-called iso-energetic or elastic approximation (cf. Bruenn 1985; Reddy et al. 1998), which is the standard simplification for calculating rates of neutral-current neutrino scatterings off free nucleons and charged-current absorption reactions on nucleons, has been abandoned in order to take into account energy exchange due to nucleon recoil and thermal motions (e.g. Schinder 1990) as well as nucleon-nucleon correlations in the dense medium (Burrows \& Sawyer 1998, 1999; Reddy et al. 1998, 1999). Modifications of the neutrino opacities due to the weak magnetism corrections associated with the large anomalous magnetic moments of the proton and the neutron are also accounted for (cf. Horowitz 2002). Moreover, we employ in the reaction cross sections a density dependent effective mass of the nucleon, which at nuclear densities is different from its vacuum value, and also take into account the possible quenching of the axial-vector coupling in nuclear matter (Carter $\&$ Prakash 2002). In the following we shall describe the numerical handling of these neutrino-nucleon interactions in some detail.

Note that the complete list of considered interactions also includes neutrino pair production by nucleon-nucleon bremsstrahlung and in particular also the flavor-coupling neutrino interactions (last two lines of Table A.1), which until recently have received only little attention from core-collapse supernova modelers. The significance of these reactions as well as implementation details were discussed elsewhere (Buras et al. 2003a). These processes are therefore not included in the following discussion.

\section{A.1. Neutrino-nucleon interactions}

\section{A.1.1. Neutrino-nucleon scattering $(v \mathrm{~N} \rightleftharpoons v \mathrm{~N})$}

Differential rates for inelastic scattering of neutrinos off free nucleons are calculated according to Eq. (38) of Burrows \& Sawyer (1998). For incorporating this type of neutrino-matter interactions into our transport code the formalism developed for inelastic scattering of neutrinos off electrons (NES) can be exploited (see Rampp \& Janka 2002, Appendix A). Different from NES, however, the angular integration (let $\omega$ denote the cosine of the scattering angle) of the scattering kernels $R\left(\epsilon, \epsilon^{\prime}, \omega\right)$ which yields the coefficients $\phi_{l}\left(\epsilon, \epsilon^{\prime}\right)$ of the corresponding Legendre expansion (cf. Bruenn 1985, Appendix C) cannot be performed analytically in the case of neutrino-nucleon scattering. Moreover, the characteristic width of the kernels as a function of inand outgoing neutrino energies $\epsilon, \epsilon^{\prime}$ can be small (but finite) compared to the numerical resolution of the neutrino spectrum in the transport scheme, in which currently only of the order of 20 energy bins with a resolution of $\Delta \epsilon / \epsilon \simeq 0.3$ can be afforded. Hence, in order to adequately sample the scattering kernels on such a coarse energy grid it is not sufficient to simply evaluate the functions $\phi_{l}\left(\epsilon, \epsilon^{\prime}\right)$ at each combination of energies $\left(\epsilon_{j+\frac{1}{2}}, \epsilon_{j^{\prime}+\frac{1}{2}}\right)$. Instead, we introduce for each energy bin $\left[\epsilon_{j}, \epsilon_{j+1}\right]$ a numerical sub-grid of $N_{\epsilon_{j}}$ neutrino energies $\epsilon_{j} \leq \epsilon \leq \epsilon_{j+1}$ (and equivalently for the final-state energies $\epsilon^{\prime}$ ), and $N_{\omega}$ angle cosines $-1 \leq \omega \leq 1$ to compute $R\left(\epsilon, \epsilon^{\prime}, \omega\right)$ as given by Burrows \& Sawyer (1998, Eq. (38)) for all such combinations of $\epsilon$, $\epsilon^{\prime}$, and $\omega$. For fixed 
values of $\epsilon$ and $\epsilon^{\prime}$ we then numerically integrate $R\left(\epsilon, \epsilon^{\prime}, \omega\right)$, appropriately weighted with the Legendre polynomials $P_{l}(\omega)$ over angles to obtain $\phi_{l}\left(\epsilon, \epsilon^{\prime}\right)$. Averaging $\phi_{l}\left(\epsilon, \epsilon^{\prime}\right)$ over all subgrid energies $\epsilon$ in energy bin $\left[\epsilon_{j}, \epsilon_{j+1}\right]$ and summing over all energies $\epsilon^{\prime}$ in bin $\left[\epsilon_{j^{\prime}}, \epsilon_{j^{\prime}+1}\right]$ the final, binned Legendre coefficients $\phi_{l}\left(\epsilon_{j+\frac{1}{2}}, \epsilon_{j^{\prime}+\frac{1}{2}}\right)$ are computed. These are then employed in our neutrino transport scheme. In practice a six-point Gauss-quadrature scheme is used here for the angular and energy integrations. In order to correctly reproduce the low-temperature (in the SN case therefore low-density) and low-neutrino energy limit, where only tiny energy transfers $\epsilon-\epsilon^{\prime}$ between neutrinos and nucleons occur and the coefficients $\phi_{l}$ become increasingly narrowly peaked functions of $\epsilon-\epsilon^{\prime}$, we calculate $\phi_{l}$ according to the (analytically tractable) iso-energetic approximation (Bruenn 1985; Reddy et al. 1998) if the density drops below $10^{8} \mathrm{~g} \mathrm{~cm}^{-3}$. Note that in this case the cross section is corrected a posteriori for nucleon recoil effects (see end of Sect. A.1.4) but non-vanishing energy exchange between high-energy neutrinos and nucleons are ignored. At densities below $10^{8} \mathrm{~g} \mathrm{~cm}^{-3}$, however, the typical neutrino energies are moderate $\left(\left\langle\epsilon_{v}\right\rangle<30 \mathrm{MeV}\right)$ and the scattering rate with nucleons becomes very low.

Given $\phi_{l}^{\text {out }}\left(\epsilon_{j}, \epsilon_{j^{\prime}}\right)$ for $\epsilon_{j} \leq \epsilon_{j^{\prime}}$, detailed balance arguments (see Cernohorsky 1994) are exploited in order to compute $\phi_{l}^{\text {out }}\left(\epsilon_{j}, \epsilon_{j^{\prime}}\right)$ for $\epsilon_{j}>\epsilon_{j^{\prime}}$ and the coefficients $\phi_{l}^{\text {in }}\left(\epsilon_{j}, \epsilon_{j^{\prime}}\right)=\phi_{l}^{\text {out }}\left(\epsilon_{j^{\prime}}, \epsilon_{j}\right)$, corresponding to the inverse reaction (for details, see Rampp \& Janka 2002, Appendix A).

Once the Legendre coefficients $\phi_{0}$ and $\phi_{1}$ are known, the contribution of neutrino-nucleon scattering to the collision integral of the Boltzmann equation and its angular moments is calculated in exactly the same way as described for NES in Rampp \& Janka (2002, Appendix A).

Note that evaluating Eq. (38) of Burrows \& Sawyer (1998) requires the knowledge of nucleon-nucleon interaction potentials. Within the framework pursued by Burrows \& Sawyer (1998) the latter can be expressed in terms of the Fermi-liquid parameters, which, in turn, are directly related to macroscopic observables such as the incompressibility modulus $K_{\mathrm{S}}$ and the symmetry energy $S_{\mathrm{v}}$ of bulk nuclear matter (see Reddy et al. 1999). Accordingly, we adopt values for the Fermi-liquid parameters which are consistent with the parameters chosen for the nuclear EoS we use for our simulations (Lattimer \& Swesty 1991, with $K_{\mathrm{s}}=$ $\left.180 \mathrm{MeV}, S_{\mathrm{v}}=29.3 \mathrm{MeV}\right)$.

\section{A.1.2. Absorption of electron-flavor neutrinos by free nucleons $\left(v_{\mathrm{e}} \mathrm{n} \rightleftharpoons \mathrm{e}^{-} \mathrm{p}, \bar{v}_{\mathrm{e}} \mathrm{p} \rightleftharpoons \mathrm{e}^{+} \mathrm{n}\right)$}

The calculation of the inverse mean free path $1 / \lambda(\epsilon)$ for absorption of electron (anti)neutrinos by free neutrons (protons) is based on Eq. (2) of Burrows \& Sawyer (1999). For the numerical implementation we employ the same techniques which we have already described for the neutral-current reactions in Sect. A.1.1. Different from neutrino-nucleon scattering, however, the outgoing lepton is a charged lepton, which, in our context is assumed to be in local thermodynamic equilibrium within the stellar medium. Hence, the dependence of the interaction kernels on the energy $\epsilon^{\prime}$ of the outgoing lepton does not need to be retained and the individual sums over energy bins $\left[\epsilon_{j^{\prime}}, \epsilon_{j^{\prime}+1}\right]$ are consequently replaced by the integral over the entire spectrum of energies $\epsilon^{\prime}$ of the outgoing charged lepton. Given $1 / \lambda(\epsilon)$, the absorption opacity corrected for stimulated absorption $\kappa^{*}(\epsilon)$ which enters our neutrino transport scheme can be calculated in a straightforward way (cf. Rampp \& Janka 2002, Appendix A).

\section{A.1.3. Effective mass of the nucleons}

Following Burrows \& Sawyer (1999) we substitute an effective mass $m^{*}$ for the nucleon mass $m$, wherever the latter appears explicitly in the formalism. Notably, $m^{*}$ is also used for inverting the relation between particle density and chemical potential which is used to compute the chemical potentials of protons and nucleons (displaced by the nuclear interaction potential) for given number densities (cf. Burrows \& Sawyer 1998, Eq. (11)).

For the effective mass we adopt the parametric density dependence (Reddy et al. 1999)

$m^{*}(\rho)=\frac{m}{1+\alpha \cdot \rho / \rho_{\text {nuc }}}$

where $m$ is the vacuum mass of the nucleon and the constant $\alpha$ is defined by the value of the effective mass at nuclear saturation density $\left(\rho_{\text {nuc }} / m=0.16 \mathrm{fm}^{-3}\right)$ being $m^{*}\left(\rho_{\text {nuc }}\right)=0.8 \mathrm{~m}$.

\section{A.1.4. Weak magnetism}

Correction factors for the iso-energetic cross-sections accounting both for the effects of weak magnetism and nucleon recoil effects are provided by Horowitz (2002). Both effects appear at the level of $O(\epsilon / m)$ with $\epsilon$ being the neutrino energy and $m$ the nucleon mass. For our purposes, we have to disentangle the contributions of both effects to the final rates in order to obtain a pure weak magnetism correction for the formalism described above, since the latter already includes recoil and also nucleon-nucleon correlations, but disregards weak magnetism. This is achieved by analytically averaging the differential rates which include weak magnetism as well as recoil and those for recoil alone over the scattering angle and using the resulting angle-independent ratio of both as a weak magnetism correction factor to the rates of Burrows \& Sawyer $(1998,1999)$. For the charged-current interactions the angular reduction is performed by simply integrating the differential rates over all directions of motion of the charged lepton 
in the final state. In case of neutral-current scattering a $(1-\omega)$-weighting for the differential rate is used which is motivated by the definition of the transport opacity (for details, see Horowitz 2002).

In detail, the Legendre coefficients for neutral-current scatterings as described in Sect. A.1.1 are modified according to $\phi_{l}^{\text {out }}\left(\epsilon, \epsilon^{\prime}\right) \rightarrow \xi^{\text {nc }}(\epsilon) \cdot \phi_{l}^{\text {out }}\left(\epsilon, \epsilon^{\prime}\right)$, where

$\xi^{\mathrm{nc}}(\epsilon) \equiv \frac{n_{\mathrm{n}} \cdot X_{\mathrm{WM}, \mathrm{Rec}}^{\mathrm{nc}, \mathrm{n}}(\epsilon)+n_{\mathrm{p}} \cdot \mathcal{X}_{\mathrm{WM}, \mathrm{Rec}}^{\mathrm{nc}: \mathrm{p}}(\epsilon)}{n_{\mathrm{n}} \cdot \mathcal{X}_{\operatorname{Rec}}^{\mathrm{ncc}}(\epsilon)+n_{\mathrm{p}} \cdot \mathcal{X}_{\operatorname{Rec}}^{\mathrm{nc}: \mathrm{R}}(\epsilon)}$

is the ratio of the full correction factor (Horowitz 2002, Eq. (32))

$$
\begin{array}{r}
\mathcal{X}_{\mathrm{WM}, \mathrm{Rec}}^{\mathrm{nc} \cdot \mathrm{n} / \mathrm{p}}(\epsilon)=\left\{C_{\mathrm{V}}^{2}\left[\frac{e-1}{2 e^{3}} \ln \zeta+\frac{3+12 e+9 e^{2}-10 e^{3}}{3 e^{2} \zeta^{3}}\right]+C_{\mathrm{A}}^{2}\left[\frac{1+e}{2 e^{3}} \ln \zeta-\frac{10 e^{3}+27 e^{2}+18 e+3}{3 e^{2} \zeta^{3}}\right]\right. \\
\pm\left(C_{\mathrm{V}}+F_{2}\right) C_{\mathrm{A}}\left[\frac{1}{e^{2}} \ln \zeta-\frac{2+10 e+\frac{28}{3} e^{2}}{e \zeta^{3}}\right]+C_{\mathrm{V}} F_{2}\left[\frac{1}{e^{2}} \ln \zeta-\frac{2}{3}\left(\frac{3+15 e+22 e^{2}}{e \zeta^{3}}\right)\right] \\
\left.+F_{2}^{2}\left[\frac{1}{4 e^{2}} \ln \zeta+\frac{8 e^{3}-22 e^{2}-15 e-3}{6 e \zeta^{3}}\right]\right\} /\left\{\frac{2}{3}\left(C_{\mathrm{V}}^{2}+5 C_{\mathrm{A}}^{2}\right)\right\}
\end{array}
$$

to the correction factor for recoil alone (Horowitz 2002, Eq. (29))

$X_{\mathrm{Rec}}^{\mathrm{nc} \mathrm{n} / \mathrm{p}}=\left\{C_{\mathrm{V}}^{2}\left[\frac{e+1}{e^{3}} \ln \zeta-\frac{2}{e^{2}}\right]+C_{\mathrm{A}}^{2}\left[\frac{2 e^{2}-1-e}{e^{3} \zeta} \ln \zeta+\frac{2}{e^{2} \zeta}\right]\right\} /\left\{\frac{2}{3}\left(C_{\mathrm{V}}^{2}+5 C_{\mathrm{A}}^{2}\right)\right\}$.

In Eqs. (A.3), (A.4) we have used the abbreviations

$e(\epsilon) \equiv \frac{\epsilon}{m c^{2}} \quad$ and $\quad \zeta(e) \equiv 1+2 e$.

Numerical values of the coupling constants $C_{\mathrm{V}}, C_{\mathrm{A}}$ and $F_{2}$ can be found in Horowitz (2002, Table I). Since the values of $C_{\mathrm{V}}, C_{\mathrm{A}}$ and $F_{2}$ are different for the reactions $v \mathrm{p} \rightleftharpoons v \mathrm{p}$ and $v \mathrm{n} \rightleftharpoons v \mathrm{n}$, the individual correction factors for protons $\mathcal{X}^{\mathrm{nc}: \mathrm{p}}$ and neutrons $\mathcal{X}^{\mathrm{nc}: \mathrm{n}}$ are weighted with the corresponding number densities $n_{\mathrm{n}}$ and $n_{\mathrm{p}}$, respectively, to yield the final correction factor $\xi^{\text {nc }}$ for neutralcurrent scatterings as defined in Eq. (A.2). This factor is then applied to the combined rate of neutrino scatterings off neutrons and protons in our code.

Analogously, we modify the inverse mean free path for charged-current reactions (see Sect. A.1.2) according to $1 / \lambda(\epsilon) \rightarrow$ $\xi^{\mathrm{cc}}(\epsilon) \cdot 1 / \lambda(\epsilon)$, where

$\xi^{\mathrm{cc}}(\epsilon) \equiv \frac{X_{\mathrm{WM}, \mathrm{Rec}}^{\mathrm{cc}}(\epsilon)}{\mathcal{X}_{\mathrm{Rec}}^{\mathrm{cc}}(\epsilon)}$

with

$$
\begin{array}{r}
X_{\mathrm{WM}, \operatorname{Rec}}^{\mathrm{cc}}(\epsilon)=\left\{C_{\mathrm{V}}^{2}\left(1+4 e+\frac{16}{3} e^{2}\right)+3 C_{\mathrm{A}}^{2}\left(1+\frac{4}{3} e\right)^{2} \pm 4\left(C_{\mathrm{V}}+F_{2}\right) C_{\mathrm{A}} e\left(1+\frac{4}{3} e\right)\right. \\
\left.+\frac{8}{3} C_{\mathrm{V}} F_{2} e^{2}+\frac{5}{3} e^{2}\left(1+\frac{2}{5} e\right) F_{2}^{2}\right\}\left\{\left\{\left(C_{\mathrm{V}}^{2}+3 C_{\mathrm{A}}^{2}\right) \zeta^{3}\right\},\right.
\end{array}
$$

as given by Horowitz (2002, Eq. (22)), and (ibid, Eq. (19))

$X_{\mathrm{Rec}}^{\mathrm{cc}}(\epsilon)=\left\{C_{\mathrm{V}}^{2}\left[\frac{1}{e}-\frac{1}{2 e^{2}} \ln \zeta\right]+C_{\mathrm{A}}^{2}\left[\frac{4 e^{2}-2 e+\zeta \ln \zeta}{2 e^{2} \zeta}\right]\right\}\left\{\left\{C_{\mathrm{V}}^{2}+3 C_{\mathrm{A}}^{2}\right\}\right.$.

The \pm -symbol in Eqs. (A.3), (A.7) translates to a positive sign for neutrinos and a negative sign for antineutrinos, respectively. In our transport code, we presently do not discriminate between $v_{\mu, \tau}$ and the corresponding antiparticles. For these flavors we therefore adopt the arithmetic mean of the correction factors of Eq. (A.3) for neutrino and antineutrino scattering which in effect means that the terms with the \pm -symbol cancel for the heavy-lepton neutrinos.

Recall that we switch from the description of Burrows \& Sawyer $(1998,1999)$ to the iso-energetic approximation (Bruenn 1985) if $\rho<10^{8} \mathrm{~g} \mathrm{~cm}^{-3}$ (see Sect. A.1.1). Consequently, in order to take into account nucleon recoil and weak magnetism also at these conditions the correction factors defined in Eqs. (A.2), (A.6) are replaced by $\xi^{\mathrm{nc}}=\left(n_{\mathrm{n}} \cdot \mathcal{X}_{\mathrm{WM}, \operatorname{Rec}}^{\mathrm{nc}: \mathrm{n}}+n_{\mathrm{p}} \cdot \mathcal{X}_{\mathrm{WM}, \operatorname{Rec}}^{\mathrm{nc} p \mathrm{p}}\right) /\left(n_{\mathrm{n}}+n_{\mathrm{p}}\right)$, and $\xi^{\mathrm{cc}}=X_{\mathrm{WM}, \mathrm{Rec}}^{\mathrm{cc}}$, respectively, whenever the density falls below $10^{8} \mathrm{~g} \mathrm{~cm}^{-3}$. 


\section{A.2. Quenching of the axial-vector coupling}

In the calculations of all neutrino-matter interactions we replace the axial-vector coupling $g_{\mathrm{A}}=1.254$ by an effective, "quenched" value $g_{\mathrm{A}}^{*}$, which depends on the baryon density according to

$g_{\mathrm{A}}^{*}(\rho)=g_{\mathrm{A}}\left(1-\frac{\rho}{4.15\left(\rho_{\mathrm{nuc}}+\rho\right)}\right)$,

as suggested by Carter \& Prakash (2002, Eq. (13)). In effect, opacities are reduced roughly by a factor $\left(g_{\mathrm{A}}^{*} / g_{\mathrm{A}}\right)^{2}$ at densities $\rho \gtrsim \rho_{\text {nuc }}$.

\section{A.3. Discussion}

Main effects: in Figs. A.1, A.2 opacities for neutrino-nucleon interactions computed with the procedures described above are compared with the iso-energetic approximation adopted by Bruenn (1985) and also with the description of Schinder (1990). The latter work approximately takes into account the reaction kinematics (recoil, thermal motions and final-state blocking of the nucleons) but disregards weak magnetism and nucleon-nucleon correlations. According to our core-collapse simulations for the $15 M_{\odot}$ star the chosen combinations of values for the density $\rho$, temperature $T$, and electron fraction $Y_{\mathrm{e}}$ of the stellar medium are characteristic for the conditions in the gain layer, where neutrino heating is strongest (top row of Figs. A.1, A.2), the region where most of the neutrino luminosity is produced (middle row), and the interior of the forming proto neutron star (bottom row).

As discussed in detail by Horowitz (2002) the effects of weak magnetism and nucleon recoil counterbalance each other for $v_{\mathrm{e}}$ while in the case of $\bar{v}_{\mathrm{e}}$ both effects add up leading to an appreciable net reduction of the standard opacities (computed according to Bruenn 1985). This can be seen in Figs. A.1, A.2 by comparing the bold, dashed lines ("elastic approximation") with the thin, dotted ("recoil only") and dash-dotted ("recoil plus weak magnetism") lines. While for given neutrino energy, the relative effects of the weak magnetism and recoil corrections are independent of the nucleon density, a sizeable additional reduction of both $v_{\mathrm{e}}$ and $\bar{v}_{\mathrm{e}}$ opacities due to nucleon-nucleon correlations shows up at nuclear and supranuclear densities (see Burrows \& Sawyer 1998, 1999; Reddy et al. 1998, 1999; Horowitz 2002, for a thorough discussion of the underlying physics).

This is also illustrated by Fig. A.4 which displays the ratio of the rates computed with the procedures described above to the iso-energetic approximations adopted from Bruenn (1985), for a typical stellar (post-bounce) profile. Using the density as a coordinate (and taking $T(\rho)$ and $Y_{\mathrm{e}}(\rho)$ from the stellar profile shown in Fig. A.3) we plot this ratio for the charged-current (left column) and neutral-current interactions (right column) as function of density for three different values of the neutrino energy. Up to densities of $10^{13} \mathrm{~g} \mathrm{~cm}^{-3}$ one observes a nearly constant ratio of the interaction rates, which is weakly dependent on the thermodynamic conditions of the stellar medium but increases for higher neutrino energies. Extant variations can be attributed to small but finite effects of the reaction kinematics (the latter are disregarded for $\rho<10^{8} \mathrm{~g} \mathrm{~cm}^{-3}$ where the ratios deviate from unity because of weak magnetism and recoil corrections of the interaction cross sections in the numerators; cf. Sects. A.1.1, A.1.4). While sizeable cross section reduction due to weak magnetism and recoil of the nucleons is visible for $\bar{v}_{\mathrm{e}}$ (dashed lines) the aforementioned counterbalancing effect leads to only small deviations from the iso-energetic approximation in case of $v_{\mathrm{e}}$ (solid lines). For densities between $10^{13} \mathrm{~g} \mathrm{~cm}^{-3}$ and $10^{14} \mathrm{~g} \mathrm{~cm}^{-3}$ the prominent peak in the adopted temperature profile of the stellar medium (see Fig. A.3) is reflected in the density variation of the interaction rates plotted in Fig. A.4 due to the temperature dependence of the reaction kinematics and nucleon-nucleon correlations. Interestingly, for low neutrino energies this leads to an increase of the cross sections compared to the iso-energetic approximation (top and middle row of Fig. A.4). In the considered density and temperature range, however, neutrinos are in thermodynamic equilibrium with the stellar medium and therefore precise values of the cross sections are not very relevant for neutrinos of low energies. In the high-energy part of the spectrum, however, which carries the significant part of the neutrino flux, our description of the neutrino-matter interactions leads to an overall reduction of the opacities for neutrinos and antineutrinos of all flavors (bottom row of Fig. A.4) and hence facilitates transport of neutrinos out of the proto neutron star.

Numerical considerations: for plotting the solid lines in Figs. A.1, A.2 the corresponding cross sections were evaluated on an equidistant energy grid consisting of 100 points. Such spectral resolution, however, can currently not be afforded in our dynamical supernova simulations. For comparison we therefore supplement the plots with values (shown by crosses) computed on the comparably coarse energy grid of the simulations (geometrical grid of 17 bins). For a wide range of conditions we find good overall agreement with the corresponding "reference" solutions. Notable deviations show up in regions with a steep variation of the opacity with the neutrino energy, which can be caused, e.g., by the final state blocking of the degenerate electron gas in the reaction $v_{\mathrm{e}}+\mathrm{n} \rightarrow \mathrm{e}^{-}+\mathrm{p}$ (see Fig. A.5). Obviously, this phenomenon is not specific to the adopted physical description (see, e.g. the open triangles in Fig. A.5) but is simply due to the fact that the rates are calculated as averages over the finite width of an energy bin (cf. Sects. A.1.1, A.1.2). Increasing the number of quadrature weights for the corresponding numerical integrations within the spectral (and angular) bins by a factor of five each, i.e. employing 30-point Gauss-quadratures (open diamonds in Figs. A.1, A.2), we find very good agreement with the standard six-point results (crosses). Hence, the relevant discretization 
R. Buras et al.: Two-dimensional hydrodynamic core-collapse supernova simulations with spectral neutrino transport. I., Online Material p 6

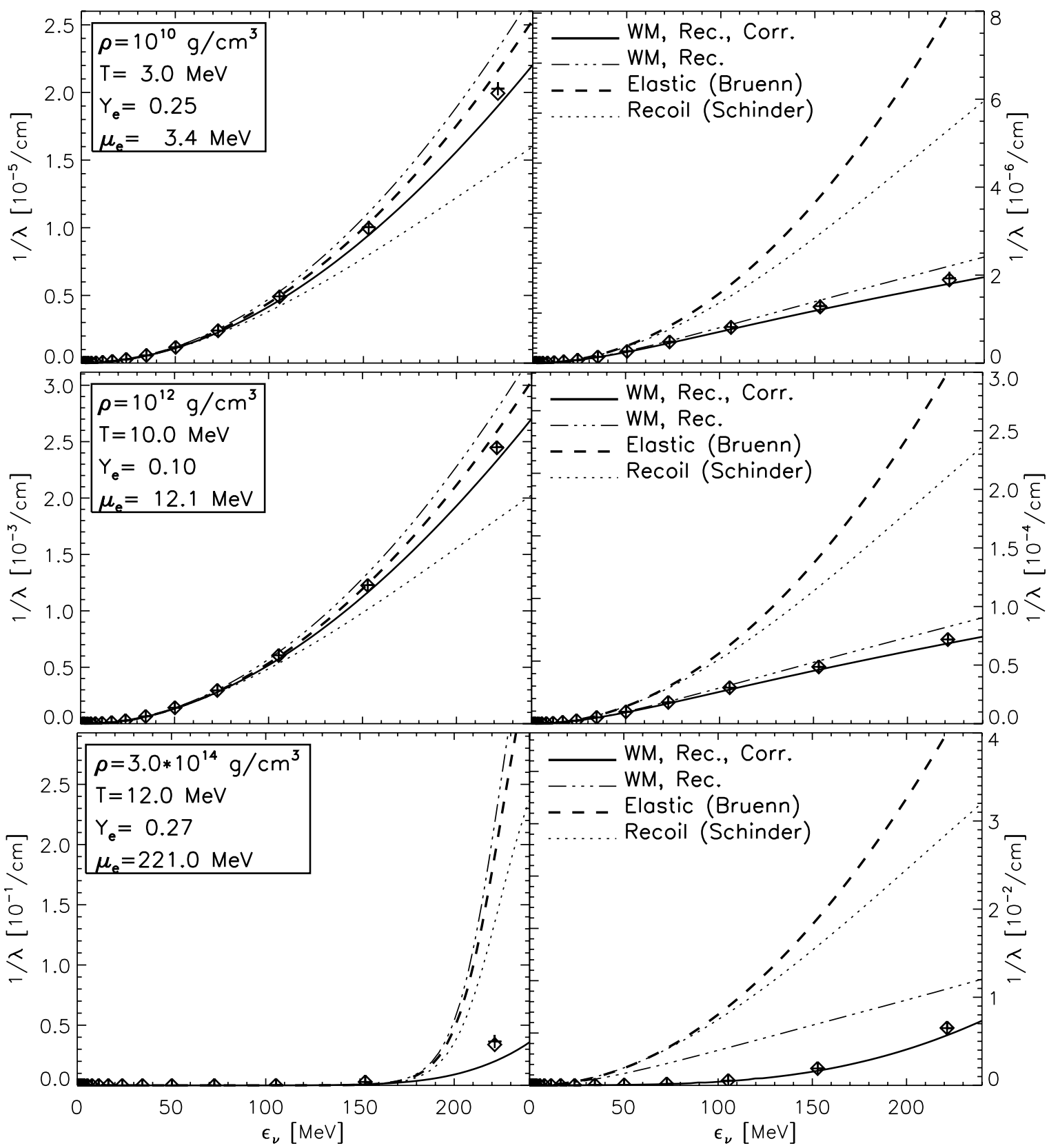

Fig. A.1. Inverse mean free paths as functions of neutrino energy for absorption of $v_{\mathrm{e}}$ (left column) and $\bar{v}_{\mathrm{e}}$ (right column) by free nucleons. Note the different scales of the ordinates. Solid lines and symbols are drawn for the charged-current reaction rates computed according to Sect. A.1.2. Thermodynamic conditions are given in the top left corners of the plots. For given density effective values for the nucleon mass $m^{*}$ and the axial-vector coupling $g_{\mathrm{A}}^{*}$ were calculated using Eqs. (A.1) and (A.9), respectively. Thin dashed-dotted lines show the weak magnetism plus recoil correction of Horowitz (2002) applied to the iso-energetic cross section of Bruenn (1985), which itself is drawn as a dashed line. The dotted lines correspond to the recoil and thermal motion approximation of Schinder (1990). In all cases the neutrino phase space was assumed to be empty. Lines interpolate values computed on an energy grid of 100 points which are equidistantly spaced between 0 and $380 \mathrm{MeV}$. For comparison, crosses show the corresponding rates on a geometrical energy grid with 17 bins and six-point Gauss-quadratures for the angular and spectral integrations (relevant for the description given in Sect. A.1.2). This is the typical spectral resolution of our dynamical supernova simulations. Results obtained by using 30-point Gauss-quadratures instead are displayed by open diamonds. See the end of Sect. A.3 for a discussion.

error of the dynamical simulations is dominated by the employed overall spectral resolution (relative width of energy bins) and not by the specific sub-gridding used for evaluating the neutrino opacities. 
R. Buras et al.: Two-dimensional hydrodynamic core-collapse supernova simulations with spectral neutrino transport. I., Online Material p 7

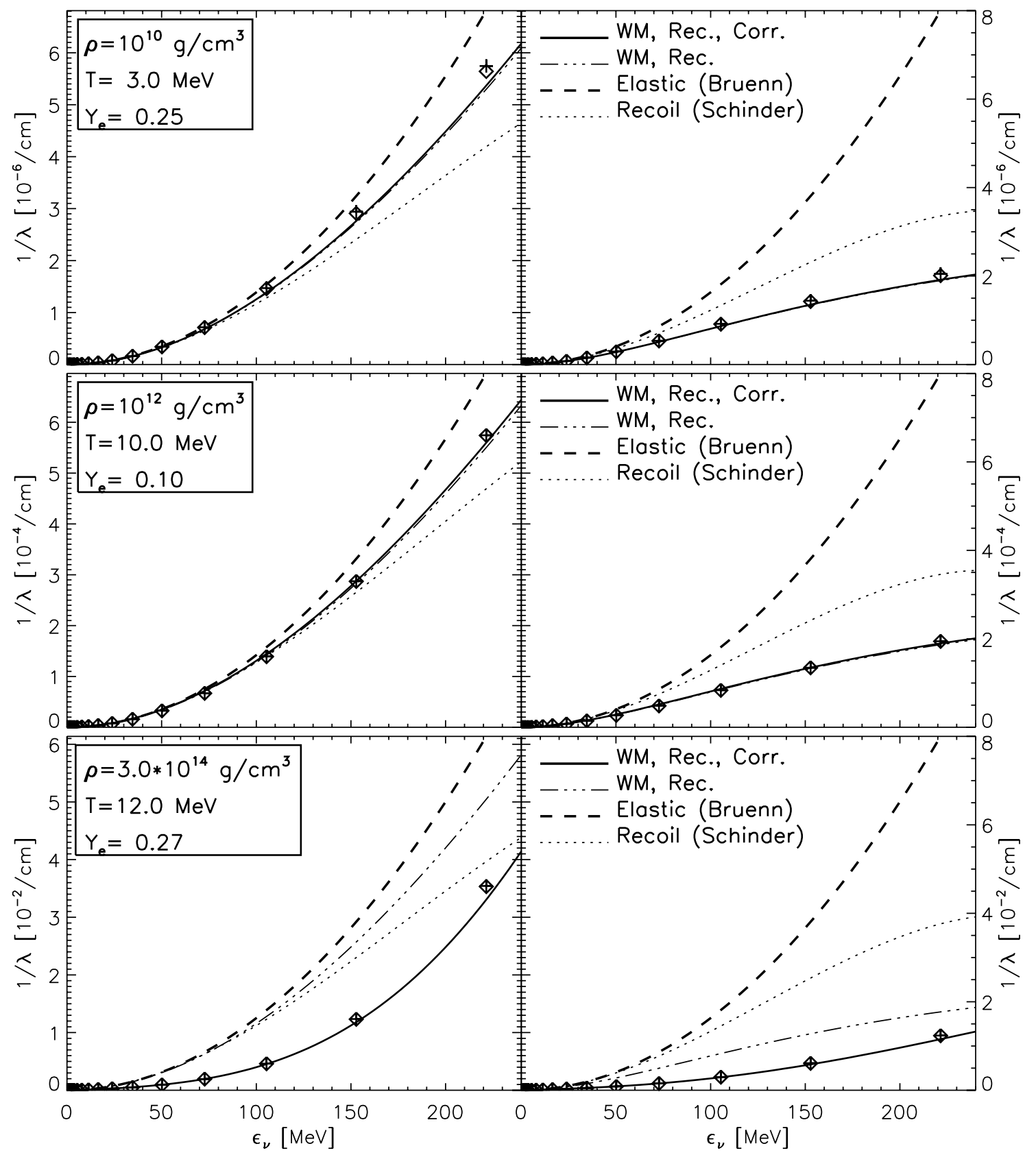

Fig. A.2. Same as Fig. A.1, but showing the inverse mean free paths for neutral-current scattering of neutrinos (left column) and antineutrinos (right column) as computed according to Sect. A.1.1 (solid lines, symbols) in comparison with the conventional ("standard") description (dashed lines) and different approximations to the complete physics (dotted and dash-dotted). In order to obtain the quantity $1 / \lambda(\epsilon)$ the differential scattering rate $R\left(\epsilon, \epsilon^{\prime}, \omega\right)$ was integrated over the spectrum of final neutrino energies $\epsilon^{\prime}$ and all values of the cosine $\omega$ of the scattering angle.

\section{Appendix B: Moments equations in three dimensions}

To order $O(v / c)$ of the fluid velocities (the so-called Newtonian approximation) the full three-dimensional moments equations are given by (Kaneko et al. 1984, correcting a number of misprints):

$\frac{\rho}{c} \frac{D}{D t}\left(\frac{J}{\rho}\right)+\boldsymbol{\nabla} \cdot \boldsymbol{H}+\frac{1}{c} \frac{\partial}{\partial t}(\boldsymbol{\beta} \cdot \boldsymbol{H})-\frac{\epsilon}{c} \frac{\partial \boldsymbol{\beta}}{\partial t} \cdot \frac{\partial \boldsymbol{H}}{\partial \epsilon}-\epsilon \boldsymbol{\nabla} \boldsymbol{\beta}: \frac{\partial \boldsymbol{P}}{\partial \epsilon}=C^{(0)}$

$\frac{\rho}{c} \frac{D}{D t}\left(\frac{\boldsymbol{H}}{\rho}\right)+\boldsymbol{\nabla} \cdot \boldsymbol{P}+\boldsymbol{H} \cdot \boldsymbol{\nabla} \boldsymbol{\beta}+\frac{1}{c} \frac{\partial}{\partial t}(\boldsymbol{\beta} \cdot \boldsymbol{P})+\frac{J}{c} \frac{\partial \boldsymbol{\beta}}{\partial t}-\frac{1}{c} \frac{\partial \boldsymbol{\beta}}{\partial t} \cdot \frac{\partial(\epsilon \boldsymbol{P})}{\partial \epsilon}-\boldsymbol{\nabla} \boldsymbol{\beta}: \frac{\partial(\epsilon \boldsymbol{N})}{\partial \epsilon}=\boldsymbol{C}^{(1)}$,

where $\beta=\boldsymbol{v} / c$. In general spherical coordinates, the moments are defined by

$J=\frac{1}{4 \pi} \iint I \mathrm{~d} \Omega, \boldsymbol{H}=\frac{1}{4 \pi} \iint I n \mathrm{~d} \Omega, \boldsymbol{P}=\frac{1}{4 \pi} \iint I n n \mathrm{~d} \Omega, N=\frac{1}{4 \pi} \iint I n n n \mathrm{~d} \Omega$. 
R. Buras et al.: Two-dimensional hydrodynamic core-collapse supernova simulations with spectral neutrino transport. I., Online Material $p 8$

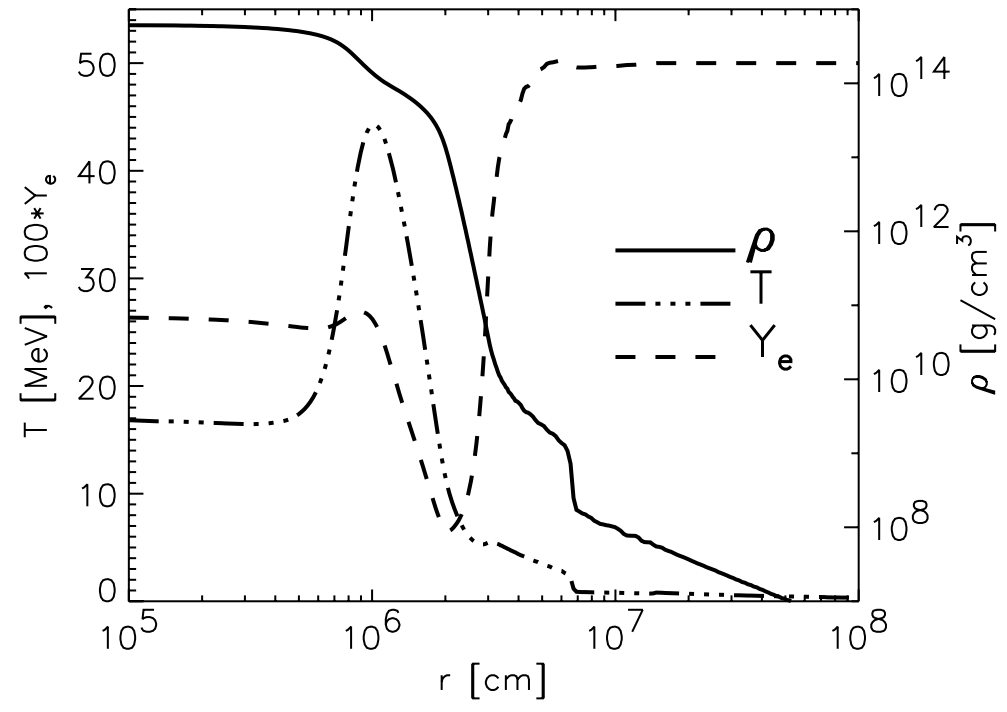

Fig. A.3. Stellar profile of a spherically symmetric postbounce model (Model s15Gio_1d.b at $t_{\mathrm{pb}}=0.257 \mathrm{~s}$ ). Density $\rho$ (solid line), temperature $T$ (dash-dotted), and electron fraction $Y_{\mathrm{e}}$ (dashed) as functions of stellar radius $r$ were used to calculate the cross sections as functions of $\rho(r)$ shown in Fig. A.4.

Here, $\boldsymbol{n}=\left(n_{r}, n_{\vartheta}, n_{\varphi}\right)=(\cos \Theta, \sin \Theta \cos \omega, \sin \Theta \sin \omega), \mu=\cos \Theta$, and $\Theta$ and $\omega$ are the two angles defining the direction of propagation. The functional dependences $\beta=\beta(t, r, \vartheta, \varphi), J=J(t, r, \vartheta, \varphi, \epsilon), \ldots$, are suppressed in the notation. The moments equations written in coordinate form are the following:

$$
\begin{aligned}
& \frac{\rho}{c} \frac{D}{D t}\left(\frac{J}{\rho}\right)+\frac{1}{r^{2}} \frac{\partial\left(r^{2} H_{r}\right)}{\partial r}+\frac{1}{r \sin \vartheta} \frac{\partial\left(\sin \vartheta H_{\vartheta}\right)}{\partial \vartheta}+\frac{1}{r \sin \vartheta} \frac{\partial H_{\varphi}}{\partial \varphi}+\frac{1}{c} \frac{\partial}{\partial t}\left(\beta_{r} H_{r}+\beta_{\vartheta} H_{\vartheta}+\beta_{\varphi} H_{\varphi}\right) \\
& -\frac{\epsilon}{c}\left(\frac{\partial \beta_{r}}{\partial t} \frac{\partial H_{r}}{\partial \epsilon}+\frac{\partial \beta_{\vartheta}}{\partial t} \frac{\partial H_{\vartheta}}{\partial \epsilon}+\frac{\partial \beta_{\varphi}}{\partial t} \frac{\partial H_{\varphi}}{\partial \epsilon}\right) \\
& -\epsilon\left\{\frac{\partial P_{r r}}{\partial \epsilon}\left(\frac{\partial \beta_{r}}{\partial r}\right)+\frac{\partial P_{\vartheta r}}{\partial \epsilon}\left(\frac{1}{r} \frac{\partial \beta_{r}}{\partial \vartheta}-\frac{\beta_{\vartheta}}{r}\right)+\frac{\partial P_{\varphi r}}{\partial \epsilon}\left(\frac{1}{r \sin \vartheta} \frac{\partial \beta_{r}}{\partial \varphi}-\frac{\beta_{\varphi}}{r}\right)+\frac{\partial P_{r \vartheta}}{\partial \epsilon}\left(\frac{\partial \beta_{\vartheta}}{\partial r}\right)+\frac{\partial P_{\vartheta \vartheta}}{\partial \epsilon}\left(\frac{1}{r} \frac{\partial \beta_{\vartheta}}{\partial \vartheta}+\frac{\beta_{r}}{r}\right)\right. \\
& \left.+\frac{\partial P_{\varphi \vartheta}}{\partial \epsilon}\left(\frac{1}{r \sin \vartheta} \frac{\partial \beta_{\vartheta}}{\partial \varphi}-\frac{\beta_{\varphi} \cos \vartheta}{r \sin \vartheta}\right)+\frac{\partial P_{r \varphi}}{\partial \epsilon}\left(\frac{\partial \beta_{\varphi}}{\partial r}\right)+\frac{\partial P_{\vartheta \varphi}}{\partial \epsilon}\left(\frac{1}{r} \frac{\partial \beta_{\varphi}}{\partial \vartheta}\right)+\frac{\partial P_{\varphi \varphi}}{\partial \epsilon}\left(\frac{1}{r \sin \vartheta} \frac{\partial \beta_{\varphi}}{\partial \varphi}+\frac{\beta_{r}}{r}+\frac{\beta_{\vartheta} \cos \vartheta}{r \sin \vartheta}\right)\right\}=C^{(0)} \\
& \frac{\rho}{c} \frac{D}{D t}\left(\frac{H_{r}}{\rho}\right)-\frac{H_{\vartheta} \beta_{\vartheta}}{r}-\frac{H_{\varphi} \beta_{\varphi}}{r}+\frac{2}{r} P_{r r}+\frac{\partial P_{r r}}{\partial r}+\frac{\cos \vartheta}{r \sin \vartheta} P_{\vartheta r}+\frac{1}{r} \frac{\partial P_{\vartheta r}}{\partial \vartheta}+\frac{1}{r \sin \vartheta} \frac{\partial P_{\varphi r}}{\partial \varphi}-\frac{P_{\vartheta \vartheta}+P_{\varphi \varphi}}{r} \\
& +H_{r}\left(\frac{\partial \beta_{r}}{\partial r}\right)+H_{\vartheta}\left(\frac{1}{r} \frac{\partial \beta_{r}}{\partial \vartheta}-\frac{\beta_{\vartheta}}{r}\right)+H_{\varphi}\left(\frac{1}{r \sin \vartheta} \frac{\partial \beta_{r}}{\partial \varphi}-\frac{\beta_{\varphi}}{r}\right) \\
& +\frac{1}{c} \frac{\partial}{\partial t}\left(\beta_{r} P_{r r}+\beta_{\vartheta} P_{\vartheta r}+\beta_{\varphi} P_{\varphi r}\right)+\frac{J}{c} \frac{\partial \beta_{r}}{\partial t}-\frac{1}{c} \frac{\partial \beta_{r}}{\partial t} \frac{\partial\left(\epsilon P_{r r}\right)}{\partial \epsilon}-\frac{1}{c} \frac{\partial \beta_{\vartheta}}{\partial t} \frac{\partial\left(\epsilon P_{\vartheta r}\right)}{\partial \epsilon}-\frac{1}{c} \frac{\partial \beta_{\varphi}}{\partial t} \frac{\partial\left(\epsilon P_{\varphi r}\right)}{\partial \epsilon} \\
& -\left\{\frac{\partial\left(\epsilon N_{r r r}\right)}{\partial \epsilon}\left(\frac{\partial \beta_{r}}{\partial r}\right)+\frac{\partial\left(\epsilon N_{r \vartheta r}\right)}{\partial \epsilon}\left(\frac{1}{r} \frac{\partial \beta_{r}}{\partial \vartheta}-\frac{\beta_{\vartheta}}{r}\right)+\frac{\partial\left(\epsilon N_{r \varphi r}\right)}{\partial \epsilon}\left(\frac{1}{r \sin \vartheta} \frac{\partial \beta_{r}}{\partial \varphi}-\frac{\beta_{\varphi}}{r}\right)\right. \\
& +\frac{\partial\left(\epsilon N_{\vartheta r r}\right)}{\partial \epsilon}\left(\frac{\partial \beta_{\vartheta}}{\partial r}\right)+\frac{\partial\left(\epsilon N_{\vartheta \vartheta r}\right)}{\partial \epsilon}\left(\frac{1}{r} \frac{\partial \beta_{\vartheta}}{\partial \vartheta}+\frac{\beta_{r}}{r}\right)+\frac{\partial\left(\epsilon N_{\vartheta \varphi r}\right)}{\partial \epsilon}\left(\frac{1}{r \sin \vartheta} \frac{\partial \beta_{\vartheta}}{\partial \varphi}-\frac{\beta_{\varphi} \cos \vartheta}{r \sin \vartheta}\right) \\
& \left.+\frac{\partial\left(\epsilon N_{\varphi r r}\right)}{\partial \epsilon}\left(\frac{\partial \beta_{\varphi}}{\partial r}\right)+\frac{\partial\left(\epsilon N_{\varphi \vartheta r}\right)}{\partial \epsilon}\left(\frac{1}{r} \frac{\partial \beta_{\varphi}}{\partial \vartheta}\right)+\frac{\partial\left(\epsilon N_{\varphi \varphi r}\right)}{\partial \epsilon}\left(\frac{1}{r \sin \vartheta} \frac{\partial \beta_{\varphi}}{\partial \varphi}+\frac{\beta_{r}}{r}+\frac{\beta_{\vartheta} \cos \vartheta}{r \sin \vartheta}\right)\right\}=C_{r}^{(1)} \text {, } \\
& \frac{\rho}{c} \frac{D}{D t}\left(\frac{H_{\vartheta}}{\rho}\right)-\frac{H_{r} \beta_{\vartheta}}{r}+\frac{H_{\varphi} \beta_{\varphi} \cos \vartheta}{r \sin \vartheta}+\frac{3}{r} P_{r \vartheta}+\frac{\partial P_{r \vartheta}}{\partial r}+\frac{\cos \vartheta}{r \sin \vartheta} P_{\vartheta \vartheta}+\frac{1}{r} \frac{\partial P_{\vartheta \vartheta}}{\partial \vartheta}+\frac{1}{r \sin \vartheta} \frac{\partial P_{\varphi \vartheta}}{\partial \varphi}-\frac{P_{\varphi \varphi} \cos \vartheta}{r \sin \vartheta} \\
& +H_{r}\left(\frac{\partial \beta_{\vartheta}}{\partial r}\right)+H_{\vartheta}\left(\frac{1}{r} \frac{\partial \beta_{\vartheta}}{\partial \vartheta}+\frac{\beta_{r}}{r}\right)+H_{\varphi}\left(\frac{1}{r \sin \vartheta} \frac{\partial \beta_{\vartheta}}{\partial \varphi}-\frac{\beta_{\varphi} \cos \vartheta}{r \sin \vartheta}\right) \\
& +\frac{1}{c} \frac{\partial}{\partial t}\left(\beta_{r} P_{r \vartheta}+\beta_{\vartheta} P_{\vartheta \vartheta}+\beta_{\varphi} P_{\varphi \vartheta}\right)+\frac{J}{c} \frac{\partial \beta_{\vartheta}}{\partial t}-\frac{1}{c} \frac{\partial \beta_{r}}{\partial t} \frac{\partial\left(\epsilon P_{r \vartheta}\right)}{\partial \epsilon}-\frac{1}{c} \frac{\partial \beta_{\vartheta}}{\partial t} \frac{\partial\left(\epsilon P_{\vartheta \vartheta}\right)}{\partial \epsilon}-\frac{1}{c} \frac{\partial \beta_{\varphi}}{\partial t} \frac{\partial\left(\epsilon P_{\varphi \vartheta}\right)}{\partial \epsilon} \\
& -\left\{\frac{\partial\left(\epsilon N_{r r \vartheta}\right)}{\partial \epsilon}\left(\frac{\partial \beta_{r}}{\partial r}\right)+\frac{\partial\left(\epsilon N_{r \vartheta \vartheta}\right)}{\partial \epsilon}\left(\frac{1}{r} \frac{\partial \beta_{r}}{\partial \vartheta}-\frac{\beta_{\vartheta}}{r}\right)+\frac{\partial\left(\epsilon N_{r \varphi \vartheta}\right)}{\partial \epsilon}\left(\frac{1}{r \sin \vartheta} \frac{\partial \beta_{r}}{\partial \varphi}-\frac{\beta_{\varphi}}{r}\right)\right. \\
& +\frac{\partial\left(\epsilon N_{\vartheta r \vartheta}\right)}{\partial \epsilon}\left(\frac{\partial \beta_{\vartheta}}{\partial r}\right)+\frac{\partial\left(\epsilon N_{\vartheta \vartheta \vartheta}\right)}{\partial \epsilon}\left(\frac{1}{r} \frac{\partial \beta_{\vartheta}}{\partial \vartheta}+\frac{\beta_{r}}{r}\right)+\frac{\partial\left(\epsilon N_{\vartheta \varphi \vartheta}\right)}{\partial \epsilon}\left(\frac{1}{r \sin \vartheta} \frac{\partial \beta_{\vartheta}}{\partial \varphi}-\frac{\beta_{\varphi} \cos \vartheta}{r \sin \vartheta}\right)
\end{aligned}
$$


R. Buras et al.: Two-dimensional hydrodynamic core-collapse supernova simulations with spectral neutrino transport. I., Online Material p 9

$\left.+\frac{\partial\left(\epsilon N_{\varphi r \vartheta}\right)}{\partial \epsilon}\left(\frac{\partial \beta_{\varphi}}{\partial r}\right)+\frac{\partial\left(\epsilon N_{\varphi \vartheta \vartheta}\right)}{\partial \epsilon}\left(\frac{1}{r} \frac{\partial \beta_{\varphi}}{\partial \vartheta}\right)+\frac{\partial\left(\epsilon N_{\varphi \varphi \vartheta}\right)}{\partial \epsilon}\left(\frac{1}{r \sin \vartheta} \frac{\partial \beta_{\varphi}}{\partial \varphi}+\frac{\beta_{r}}{r}+\frac{\beta_{\vartheta} \cos \vartheta}{r \sin \vartheta}\right)\right\}=C_{\vartheta}^{(1)}$,

$\frac{\rho}{c} \frac{D}{D t}\left(\frac{H_{\varphi}}{\rho}\right)-\frac{H_{r} \beta_{\varphi}}{r}-\frac{H_{\vartheta} \beta_{\varphi} \cos \vartheta}{r \sin \vartheta}+\frac{3}{r} P_{r \varphi}+\frac{\partial P_{r \varphi}}{\partial r}+\frac{2 \cos \vartheta}{r \sin \vartheta} P_{\vartheta \varphi}+\frac{1}{r} \frac{\partial P_{\vartheta \varphi}}{\partial \vartheta}+\frac{1}{r \sin \vartheta} \frac{\partial P_{\varphi \varphi}}{\partial \varphi}$

$+H_{r}\left(\frac{\partial \beta_{\varphi}}{\partial r}\right)+H_{\vartheta}\left(\frac{1}{r} \frac{\partial \beta_{\varphi}}{\partial \vartheta}\right)+H_{\varphi}\left(\frac{1}{r \sin \vartheta} \frac{\partial \beta_{\varphi}}{\partial \varphi}+\frac{\beta_{r}}{r}+\frac{\beta_{\vartheta} \cos \vartheta}{r \sin \vartheta}\right)$

$+\frac{1}{c} \frac{\partial}{\partial t}\left(\beta_{r} P_{r \varphi}+\beta_{\vartheta} P_{\vartheta \varphi}+\beta_{\varphi} P_{\varphi \varphi}\right)+\frac{J}{c} \frac{\partial \beta_{\varphi}}{\partial t}-\frac{1}{c} \frac{\partial \beta_{r}}{\partial t} \frac{\partial\left(\epsilon P_{r \varphi}\right)}{\partial \epsilon}-\frac{1}{c} \frac{\partial \beta_{\vartheta}}{\partial t} \frac{\partial\left(\epsilon P_{\vartheta \varphi}\right)}{\partial \epsilon}-\frac{1}{c} \frac{\partial \beta_{\varphi}}{\partial t} \frac{\partial\left(\epsilon P_{\varphi \varphi}\right)}{\partial \epsilon}$

$-\left\{\frac{\partial\left(\epsilon N_{r r \varphi}\right)}{\partial \epsilon}\left(\frac{\partial \beta_{r}}{\partial r}\right)+\frac{\partial\left(\epsilon N_{r \vartheta \varphi}\right)}{\partial \epsilon}\left(\frac{1}{r} \frac{\partial \beta_{r}}{\partial \vartheta}-\frac{\beta_{\vartheta}}{r}\right)+\frac{\partial\left(\epsilon N_{r \varphi \varphi}\right)}{\partial \epsilon}\left(\frac{1}{r \sin \vartheta} \frac{\partial \beta_{r}}{\partial \varphi}-\frac{\beta_{\varphi}}{r}\right)\right.$

$+\frac{\partial\left(\epsilon N_{\vartheta r \varphi}\right)}{\partial \epsilon}\left(\frac{\partial \beta_{\vartheta}}{\partial r}\right)+\frac{\partial\left(\epsilon N_{\vartheta \vartheta \varphi}\right)}{\partial \epsilon}\left(\frac{1}{r} \frac{\partial \beta_{\vartheta}}{\partial \vartheta}+\frac{\beta_{r}}{r}\right)+\frac{\partial\left(\epsilon N_{\vartheta \varphi \varphi}\right)}{\partial \epsilon}\left(\frac{1}{r \sin \vartheta} \frac{\partial \beta_{\vartheta}}{\partial \varphi}-\frac{\beta_{\varphi} \cos \vartheta}{r \sin \vartheta}\right)$

$\left.+\frac{\partial\left(\epsilon N_{\varphi r \varphi}\right)}{\partial \epsilon}\left(\frac{\partial \beta_{\varphi}}{\partial r}\right)+\frac{\partial\left(\epsilon N_{\varphi \vartheta \varphi}\right)}{\partial \epsilon}\left(\frac{1}{r} \frac{\partial \beta_{\varphi}}{\partial \vartheta}\right)+\frac{\partial\left(\epsilon N_{\varphi \varphi \varphi}\right)}{\partial \epsilon}\left(\frac{1}{r \sin \vartheta} \frac{\partial \beta_{\varphi}}{\partial \varphi}+\frac{\beta_{r}}{r}+\frac{\beta_{\vartheta} \cos \vartheta}{r \sin \vartheta}\right)\right\}=C_{\varphi}^{(1)}$.

Here $\beta_{r}(t, r, \vartheta, \varphi)=v_{r} / c, \beta_{\vartheta}(t, r, \vartheta, \varphi)=v_{\vartheta} / c$, and $\beta_{\varphi}(t, r, \vartheta, \varphi)=v_{\varphi} / c$. Note that using the continuity equation yields

$\frac{\rho}{c} \frac{D}{D t}\left(\frac{X}{\rho}\right)=\left(\frac{1}{c} \frac{\partial}{\partial t}+\boldsymbol{\beta} \cdot \boldsymbol{\nabla}\right) X+X \boldsymbol{\nabla} \cdot \boldsymbol{\beta}$

$$
=\left(\frac{1}{c} \frac{\partial}{\partial t}+\beta_{r} \frac{\partial}{\partial r}+\frac{\beta_{\vartheta}}{r} \frac{\partial}{\partial \vartheta}+\frac{\beta_{\varphi}}{r} \frac{\partial}{\partial \varphi}\right) X+X\left(\frac{1}{r^{2}} \frac{\partial\left(r^{2} \beta_{r}\right)}{\partial r}+\frac{1}{r \sin \vartheta} \frac{\partial\left(\sin \vartheta \beta_{\vartheta}\right)}{\partial \vartheta}+\frac{1}{r \sin \vartheta} \frac{\partial \beta_{\varphi}}{\partial \varphi}\right) .
$$

The different moments defined in Eqs. (B.3) are linked by the following relations, which are generally valid:

$$
\begin{aligned}
P_{r r}+P_{\vartheta \vartheta}+P_{\varphi \varphi} & =J, \\
N_{r r r}+N_{r \vartheta \vartheta}+N_{r \varphi \varphi} & =H_{r}, \\
N_{r r \vartheta}+N_{\vartheta \vartheta \vartheta}+N_{\vartheta \varphi \varphi} & =H_{\vartheta}, \\
N_{r r \varphi}+N_{\vartheta \vartheta \varphi}+N_{\varphi \varphi \varphi} & =H_{\varphi} .
\end{aligned}
$$

Also note that $\boldsymbol{P}$ and $\boldsymbol{N}$ are completely symmetric tensors, i.e. the indices can be permutated arbitrarily. The number of independent variables therefore is \# $(J, H, P, N)=(1,3,5,7)$ after applying Eqs. (B.9).

In two dimensions the symmetry relation $I(-\omega, \mu)=I(\omega, \mu)$ holds which leads to

$H_{\varphi}=P_{r \varphi}=P_{\vartheta \varphi}=N_{r r \varphi}=N_{r \vartheta \varphi}=N_{\vartheta \vartheta \varphi}=N_{\varphi \varphi \varphi} \equiv 0$

and therefore $\#(J, H, P, N)=(1,2,3,4)$; note that the last equation in (B.9) becomes redundant. In one dimension one has $I(\omega, \mu)=I(\mu)$ so that in addition

$H_{\vartheta}=P_{r \vartheta}=N_{r r \vartheta}=N_{\vartheta \vartheta \vartheta}=N_{\vartheta \varphi \varphi} \equiv 0$.

This time, the third equation in (B.9) becomes redundant. Furthermore, one obtains two new equations from comparing the relations in Eqs. (B.3):

$P_{\vartheta \vartheta}=P_{\varphi \varphi}, \quad N_{r \vartheta \vartheta}=N_{r \varphi \varphi}$

which results in $\#(J, H, P, N)=(1,1,1,1)$. Now one can introduce the definitions $H \equiv H_{r}, K \equiv P_{r r}, L \equiv N_{r r r}$.

In our approximation of two-dimensional transport, i.e. assuming $I(\omega, \mu)=I(\mu)$, Eqs. (B.9)-(B.12) apply, but in contrast to the one-dimensional case not all terms with lateral gradients or components vanish. The moments equations for the remaining independent variables, $J$ and $H \equiv H_{r}$, simplify from Eqs. (B.4) and (B.5) to

$$
\begin{aligned}
& \left(\frac{1}{c} \frac{\partial}{\partial t}+\beta_{r} \frac{\partial}{\partial r}+\frac{\boldsymbol{\beta}_{\boldsymbol{\vartheta}}}{\boldsymbol{r}} \frac{\boldsymbol{\partial}}{\boldsymbol{\partial \vartheta}}\right) J+J\left(\frac{1}{r^{2}} \frac{\partial\left(r^{2} \beta_{r}\right)}{\partial r}+\frac{\mathbf{1}}{\boldsymbol{r} \sin \boldsymbol{\vartheta}} \frac{\partial\left(\sin \boldsymbol{\vartheta} \boldsymbol{\beta}_{\boldsymbol{\vartheta}}\right)}{\partial \boldsymbol{\vartheta}}\right)+\frac{1}{r^{2}} \frac{\partial\left(r^{2} H\right)}{\partial r}+\frac{\beta_{r}}{c} \frac{\partial H}{\partial t} \\
& -\frac{\partial}{\partial \epsilon}\left\{\epsilon\left[\frac{1}{c} \frac{\partial \beta_{r}}{\partial t} H+K\left(\frac{\partial \beta_{r}}{\partial r}-\frac{\beta_{r}}{r}-\frac{\mathbf{1}}{\mathbf{2 r} \sin \boldsymbol{\vartheta}} \frac{\partial\left(\sin \boldsymbol{\vartheta} \boldsymbol{\beta}_{\boldsymbol{\vartheta}}\right)}{\partial \boldsymbol{\vartheta}}\right)+J\left(\frac{\beta_{r}}{r}+\frac{\mathbf{1}}{\mathbf{2 r} \sin \boldsymbol{\vartheta}} \frac{\partial\left(\sin \boldsymbol{\vartheta} \boldsymbol{\beta}_{\boldsymbol{\vartheta}}\right)}{\partial \boldsymbol{\vartheta}}\right)\right]\right\} \\
& +K\left(\frac{\partial \beta_{r}}{\partial r}-\frac{\beta_{r}}{r}-\frac{\mathbf{1}}{\mathbf{2 r} \sin \boldsymbol{\vartheta}} \frac{\partial\left(\sin \boldsymbol{\vartheta} \boldsymbol{\beta}_{\boldsymbol{\vartheta}}\right)}{\partial \boldsymbol{\vartheta}}\right)+J\left(\frac{\beta_{r}}{r}+\frac{\mathbf{1}}{\mathbf{2 r} \sin \boldsymbol{\vartheta}} \frac{\partial\left(\sin \boldsymbol{\vartheta} \boldsymbol{\beta}_{\boldsymbol{\vartheta}}\right)}{\partial \boldsymbol{\vartheta}}\right)+\frac{2}{c} \frac{\partial \beta_{r}}{\partial t} H=C^{(0)}, \\
& \left(\frac{1}{c} \frac{\partial}{\partial t}+\beta_{r} \frac{\partial}{\partial r}+\frac{\boldsymbol{\beta}_{\boldsymbol{\vartheta}}}{\boldsymbol{r}} \frac{\boldsymbol{\partial}}{\partial \boldsymbol{\vartheta}}\right) H+H\left(\frac{1}{r^{2}} \frac{\partial\left(r^{2} \beta_{r}\right)}{\partial r}+\frac{\mathbf{1}}{\boldsymbol{r} \sin \boldsymbol{\vartheta}} \frac{\partial\left(\sin \boldsymbol{\vartheta} \boldsymbol{\beta}_{\boldsymbol{\vartheta}}\right)}{\partial \boldsymbol{\vartheta}}\right)+\frac{\partial K}{\partial r}+\frac{3 K-J}{r}+H\left(\frac{\partial \beta_{r}}{\partial r}\right)+\frac{\beta_{r}}{c} \frac{\partial K}{\partial t} \\
& -\frac{\partial}{\partial \epsilon}\left\{\epsilon\left[\frac{1}{c} \frac{\partial \beta_{r}}{\partial t} K+L\left(\frac{\partial \beta_{r}}{\partial r}-\frac{\beta_{r}}{r}-\frac{1}{2 r \sin \boldsymbol{\vartheta}} \frac{\partial\left(\sin \boldsymbol{\vartheta} \boldsymbol{\beta}_{\boldsymbol{\vartheta}}\right)}{\partial \boldsymbol{\vartheta}}\right)+H\left(\frac{\beta_{r}}{r}+\frac{1}{2 r \sin \boldsymbol{\vartheta}} \frac{\partial\left(\sin \boldsymbol{\vartheta} \boldsymbol{\beta}_{\boldsymbol{\vartheta}}\right)}{\partial \boldsymbol{\vartheta}}\right)\right]\right\}+\frac{1}{c} \frac{\partial \beta_{r}}{\partial t}(J+K)=C_{r}^{(1)} .
\end{aligned}
$$


R. Buras et al.: Two-dimensional hydrodynamic core-collapse supernova simulations with spectral neutrino transport. I., Online Material p 10

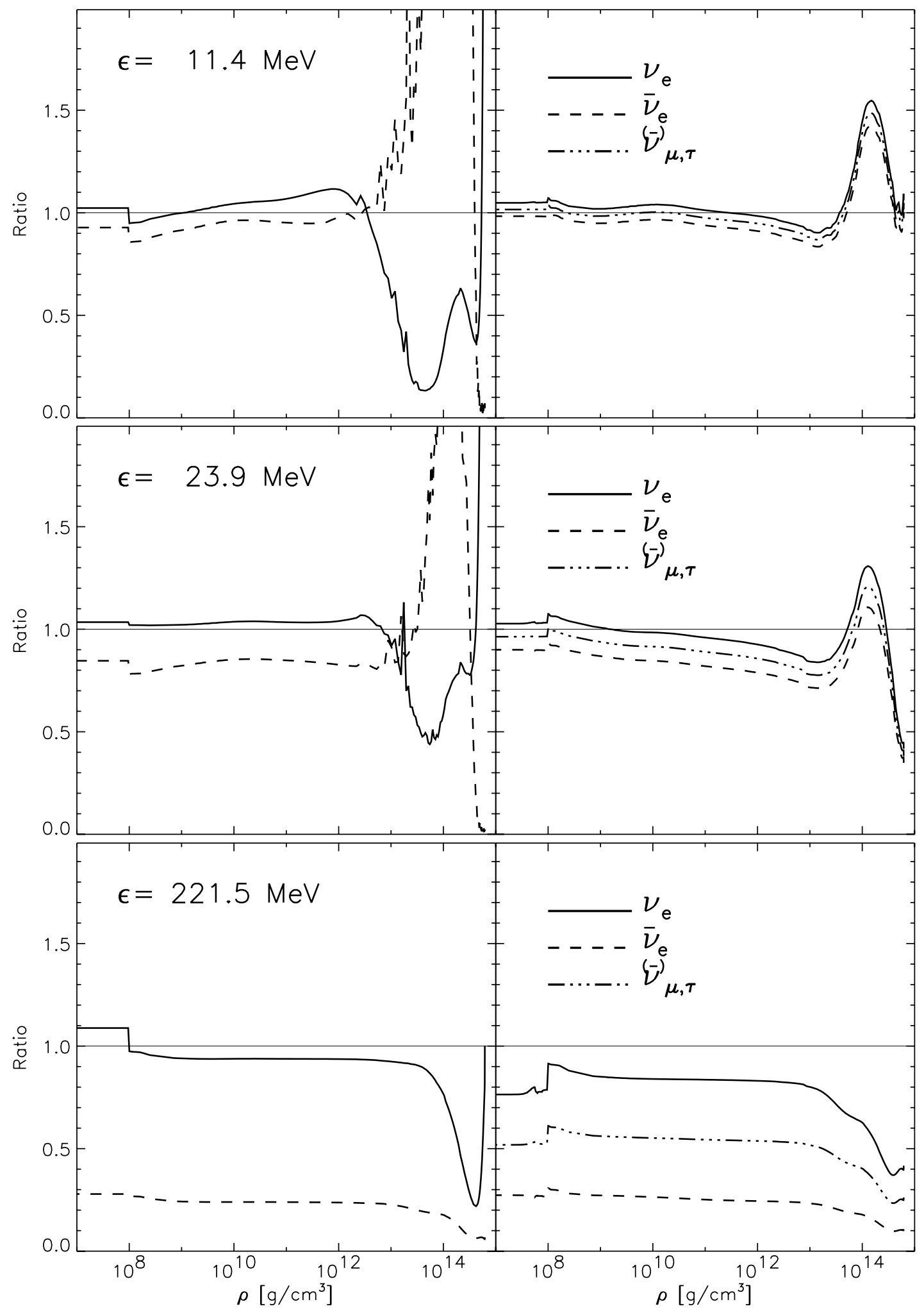

Fig. A.4. Ratios of cross sections as computed according to Sects. A.1, A.2 to the conventional ("standard") approximation for given neutrino energies as functions of density $\rho$ (the input quantities $T(\rho)$ and $Y_{\mathrm{e}}(\rho)$ were obtained from the stellar profile shown in Fig. A.3). In the left column we show the ratio for charged-current absorption of $v_{\mathrm{e}}$ (solid lines) and $\bar{v}_{\mathrm{e}}$ (dashed lines). The right column shows the ratio for neutral-current scattering of $v_{\mathrm{e}}, \bar{v}_{\mathrm{e}}$, and heavy-lepton neutrinos (dash-dotted lines) off free nucleons. According to our equal treatment of heavy-lepton neutrinos and antineutrinos the latter quantity is computed using arithmetic averages of the particle and antiparticle cross sections (see Sect. A.1.4). The neutrino phase space was assumed to be empty. 


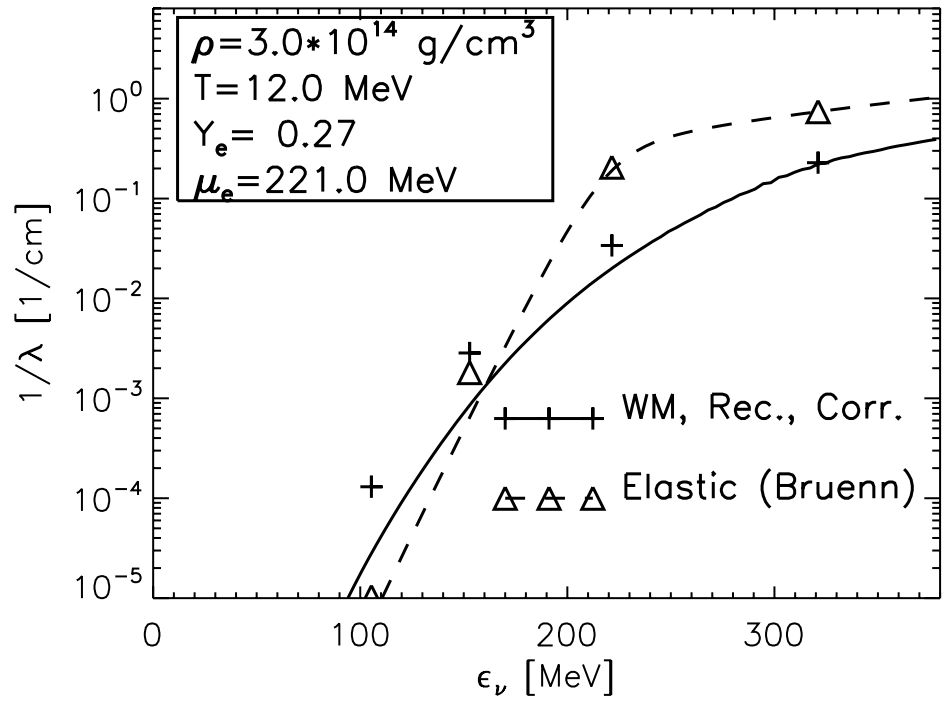

Fig. A.5. Inverse mean free path for the absorption of $v_{\mathrm{e}}$ by free neutrons for the electron-degenerate conditions given in the last row of Fig. A.1. The reference curve (solid line) was obtained with high overall spectral resolution whereas crosses show averages over the bins of a comparably coarse energy grid which is typically used in our dynamical simulations. For comparison the same averaging procedure was also applied to the iso-energetic approximation of Bruenn (1985). The results are shown as a dashed line with open triangles marking the corresponding coarse-grid average values.

With $\mathcal{J}=J / \epsilon, \mathcal{H}=H / \epsilon, \mathcal{K}=K / \epsilon$, and $\mathcal{L}=L / \epsilon$, the moments equations describing the evolution of neutrino number read

$$
\begin{aligned}
& \left(\frac{1}{c} \frac{\partial}{\partial t}+\beta_{r} \frac{\partial}{\partial r}+\frac{\boldsymbol{\beta}_{\boldsymbol{\vartheta}}}{\boldsymbol{r}} \frac{\boldsymbol{\partial}}{\partial \boldsymbol{\vartheta}}\right) \mathcal{J}+\mathcal{J}\left(\frac{1}{r^{2}} \frac{\partial\left(r^{2} \beta_{r}\right)}{\partial r}+\frac{\mathbf{1}}{\boldsymbol{r} \sin \boldsymbol{\vartheta}} \frac{\partial\left(\sin \boldsymbol{\vartheta} \boldsymbol{\beta}_{\boldsymbol{\vartheta}}\right)}{\partial \boldsymbol{\vartheta}}\right)+\frac{1}{r^{2}} \frac{\partial\left(r^{2} \mathcal{H}\right)}{\partial r}+\frac{\beta_{r}}{c} \frac{\partial \mathcal{H}}{\partial t} \\
& -\frac{\partial}{\partial \epsilon}\left\{\epsilon\left[\frac{1}{c} \frac{\partial \beta_{r}}{\partial t} \mathcal{H}+\mathcal{K}\left(\frac{\partial \beta_{r}}{\partial r}-\frac{\beta_{r}}{r}-\frac{1}{2 r \sin \boldsymbol{\vartheta}} \frac{\partial\left(\sin \boldsymbol{\vartheta} \boldsymbol{\beta}_{\boldsymbol{\vartheta}}\right)}{\partial \boldsymbol{\vartheta}}\right)+\mathcal{J}\left(\frac{\beta_{r}}{r}+\frac{1}{2 r \sin \boldsymbol{\vartheta}} \frac{\partial\left(\sin \boldsymbol{\vartheta} \boldsymbol{\beta}_{\boldsymbol{\vartheta}}\right)}{\partial \boldsymbol{\vartheta}}\right)\right]\right\}+\frac{1}{c} \frac{\partial \beta_{r}}{\partial t} \mathcal{H}=C^{(0)} \text {, } \\
& \left(\frac{1}{c} \frac{\partial}{\partial t}+\beta_{r} \frac{\partial}{\partial r}+\frac{\boldsymbol{\beta}_{\boldsymbol{\vartheta}}}{\boldsymbol{r}} \frac{\partial}{\partial \boldsymbol{\vartheta}}\right) \mathcal{H}+\mathcal{H}\left(\frac{1}{r^{2}} \frac{\partial\left(r^{2} \beta_{r}\right)}{\partial r}+\frac{1}{r \sin \boldsymbol{\vartheta}} \frac{\partial\left(\sin \boldsymbol{\vartheta} \boldsymbol{\beta}_{\boldsymbol{\vartheta}}\right)}{\partial \boldsymbol{\vartheta}}\right)+\frac{\partial \mathcal{K}}{\partial r}+\frac{3 \mathcal{K}-\mathcal{J}}{r}+\mathcal{H}\left(\frac{\partial \beta_{r}}{\partial r}\right)+\frac{\beta_{r}}{c} \frac{\partial \mathcal{K}}{\partial t} \\
& -\frac{\partial}{\partial \epsilon}\left\{\epsilon\left[\frac{1}{c} \frac{\partial \beta_{r}}{\partial t} \mathcal{K}+\mathcal{L}\left(\frac{\partial \beta_{r}}{\partial r}-\frac{\beta_{r}}{r}-\frac{1}{2 r \sin \vartheta} \frac{\partial\left(\sin \vartheta \beta_{\vartheta}\right)}{\partial \vartheta}\right)+\mathcal{H}\left(\frac{\beta_{r}}{r}+\frac{1}{2 r \sin \vartheta} \frac{\partial\left(\sin \vartheta \beta_{\vartheta}\right)}{\partial \vartheta}\right)\right]\right\} \\
& -\mathcal{L}\left(\frac{\partial \beta_{r}}{\partial r}-\frac{\beta_{r}}{r}-\frac{1}{2 r \sin \vartheta} \frac{\partial\left(\sin \vartheta \boldsymbol{\beta}_{\vartheta}\right)}{\partial \vartheta}\right)-\mathcal{H}\left(\frac{\beta_{r}}{r}+\frac{1}{2 r \sin \vartheta} \frac{\partial\left(\sin \vartheta \boldsymbol{\beta}_{\vartheta}\right)}{\partial \vartheta}\right)+\frac{1}{c} \frac{\partial \beta_{r}}{\partial t} \mathcal{T}=C_{r}^{(1)} \text {. }
\end{aligned}
$$

This system of moments Eqs. (B.13)-(B.16) is very similar to the Newtonian, $O(v / c)$ moments equations in spherical symmetry (see Rampp \& Janka 2002, Eqs. (7), (8), (30), (31)). We have set the additional terms arising from our approximative generalization to two dimensions in boldface.

\section{Appendix C: Specific energy without offset}

Here we present our new procedure how the Eulerian equations of hydrodynamics, Eqs. (1)-(7), together with a general EoS that handles NSE as well as non-NSE conditions, can be numerically treated without producing spurious local fluctuations in dependent thermodynamic variables like the entropy and pressure, a kind of numerical noise which was described in Sect. 2.5 .

As discussed in that section, the wiggles observed in radial profiles are linked to situations where the value of $\widetilde{\Gamma}_{e}=p /(e \rho)+1$ drops below 4/3. The Riemann solver in our PROMETHEUS code, however, expects $\widetilde{\Gamma}_{e}$ to be thermodynamically consistent with the adiabatic index $\Gamma_{\text {ad }}=(\mathrm{d} \ln p / \mathrm{d} \ln \rho)_{s}$, in particular $\widetilde{\Gamma}_{e}$ should fulfill $\widetilde{\Gamma}_{e} \geq 4 / 3$, which implies that $p$ and $e$ should go to zero at the same time. This, however, cannot be guaranteed with an arbitrary normalization of the energy $e$. On the other hand, there is freedom of choosing the normalization of the specific energy in the EoS, and also the solution of the time-evolution equations of a system depends only on differences of the specific energy and not on the adopted zero point of this energy. In order to employ an EoS which makes use of this natural freedom of the energy normalization in our hydrodynamics code, we therefore introduce a shift of the reference level of the energy when the latter is transferred between hydrodynamics code and EoS.

We find that the wiggles observed in the radial profiles of dependent thermodynamic variables disappear when the total specific energy used in the hydrodynamics solver PROMETHEUS is redefined compared to Sect. 2.5 as

$\varepsilon=e_{\mathrm{int}}+e_{\mathrm{kin}}$,

where $e_{\text {int }}$ is the internal specific energy and $e_{\text {kin }}=\frac{1}{2}\left(v_{r}^{2}+v_{\vartheta}^{2}+v_{\varphi}^{2}\right)$ is the kinetic energy per unit of mass. Then the EoS index $\Gamma_{e} \equiv p /\left[\left(\varepsilon-e_{\text {kin }}\right) \rho\right]+1=p /\left[e_{\text {int }} \rho\right]+1$ adopts values in the physically meaningful range $\left(\Gamma_{e} \geq 4 / 3\right)$, which seems necessary for the Riemann solver used in PROMETHEUS to produce smooth hydrodynamic solutions. However, our high-density EoS, 
provided by Lattimer \& Swesty (1991), employs an energy definition which includes the nucleon rest masses and the rest masses of the electrons, the so-called "relativistic" specific energy, plus a constant energy offset:

$e_{\mathrm{rel}, 0}=e_{\mathrm{int}}+e_{\mathrm{rm}}+e_{0}$,

where the total specific rest mass energy is defined as

$e_{\mathrm{rm}}=\sum_{k=1}^{n_{Y}} Y_{k} \cdot \frac{m_{k}}{m_{\mathrm{by}}} \cdot c^{2}+Y_{\mathrm{e}} \cdot \frac{m_{\mathrm{e}}}{m_{\mathrm{by}}} \cdot c^{2}$

The specific energy offset $e_{0}$ is an arbitrary constant (set equal to $-930.7731 \mathrm{MeV} \times 1.602 \times 10^{-6} \frac{\mathrm{erg}}{\mathrm{MeV}} / m_{\mathrm{by}}$, see Lattimer \& Swesty 1991 for details). This energy definition was used in the equation of energy solved in our hydrodynamics code hitherto, i.e. $\varepsilon=e_{\text {rel, }, 0}+e_{\text {kin }}$. For reasons explained below, we still wish to retain the energy definition, Eq. (C.2), when evaluating the EoS and at the same time use the new definition Eq. (C.1) when solving the hydrodynamics. This is achieved by the following scheme.

The Euler equation for the specific energy as defined in Eq. (C.1) can be derived from the Euler equation for the specific energy as defined in our code hitherto, Eq. (5), by subtracting the normalized sum over the Eqs. (7) and further subtracting Eq. (6), i.e. "(Eq. 5) $-\sum_{k}$ (Eq. 7) ${ }_{k} m_{k} c^{2} / m_{\text {by }}-$ (Eq. 6) $m_{e} c^{2} / m_{\text {by }}$ ":

$\frac{\partial}{\partial t}(\rho \varepsilon)+\frac{1}{r^{2}} \frac{\partial}{\partial r}\left(r^{2}(\rho \varepsilon+p) v_{r}\right)+\frac{1}{r \sin \vartheta} \frac{\partial}{\partial \vartheta}\left((\rho \varepsilon+p) \sin \vartheta v_{\vartheta}\right)=-\rho\left(v_{r} \frac{\partial \Phi}{\partial r}+\frac{v_{\vartheta}}{r} \frac{\partial \Phi}{\partial \vartheta}\right)+Q_{\mathrm{E}}+Q_{\mathrm{nuc}}$,

where the nuclear source term is

$Q_{\mathrm{nuc}}=-\sum_{k=1}^{n_{Y}} \frac{m_{k}}{m_{\mathrm{by}}} c^{2} R_{k}-\frac{m_{\mathrm{e}}}{m_{\mathrm{by}}} c^{2} Q_{\mathrm{N}}$.

The abundance of each nuclear species $k$ evolves according to Eq. (7):

$\frac{\partial}{\partial t}\left(\rho Y_{k}\right)+\frac{1}{r^{2}} \frac{\partial}{\partial r}\left(r^{2} \rho Y_{k} v_{r}\right)+\frac{1}{r \sin \vartheta} \frac{\partial}{\partial \vartheta}\left(\sin \vartheta \rho Y_{k} v_{\vartheta}\right)=R_{k}$

and the electron fraction according to Eq. (6):

$\frac{\partial}{\partial t}\left(\rho Y_{\mathrm{e}}\right)+\frac{1}{r^{2}} \frac{\partial}{\partial r}\left(r^{2} \rho Y_{\mathrm{e}} v_{r}\right)+\frac{1}{r \sin \vartheta} \frac{\partial}{\partial \vartheta}\left(\sin \vartheta \rho Y_{\mathrm{e}} v_{\vartheta}\right)=Q_{\mathrm{N}}$.

Starting at time step $n$, we calculate a hydrodynamics time step with PROMETHEUS using the initial total specific energy at this time, $\varepsilon^{n}$, as defined in Eq. (C.1). Remember that PROMETHEUS only solves the LHS of the hydrodynamics equations (in this implementation Eqs. (1)-(4), (C.4), (C.6), (C.7)), i.e. the source terms and gravitational effects on the RHS of the equations are ignored for the moment. Also note that the EoS is not solved simultaneously with the hydrodynamics so that for instance composition changes, even in regions with NSE, originate solely from advection, Eqs. (C.6)!

The solution of the evolution equations, after additionally applying the gravitational effects and all source terms except for $Q_{\text {nuc }}, Q_{\mathrm{N}}$, and $R_{k}$ yields $\varepsilon^{*}$ as well as the advected nuclear abundances $Y_{k}^{*}$ and the electron fraction $Y_{\mathrm{e}}^{*}$. The asterisk denotes that the nuclear reaction effects and the neutrino source term $Q_{\mathrm{N}}$ of time step $n+1$ have not yet been taken into account.

Next, we transform the energy to

$e_{\mathrm{rel}, 0}^{*}=\varepsilon^{*}-e_{\mathrm{kin}}^{n+1}+\sum_{k=1}^{n_{Y}} Y_{k}^{*} \cdot \frac{m_{k}}{m_{\mathrm{by}}} \cdot c^{2}+Y_{\mathrm{e}}^{*} \cdot \frac{m_{\mathrm{e}}}{m_{\mathrm{by}}} \cdot c^{2}+e_{0}$,

where $e_{\mathrm{kin}}^{n+1}$ is the specific kinetic energy after the hydrodynamics step.

This energy transformation brings us back to the energy definition originally used in Eq. (5) so that the remaining source terms of that equation and in the lepton number and nuclear abundance equations can now be applied to the hydrodynamics variables. Then all thermodynamic quantities and the composition can be updated by applying the EoS and nuclear burning routines exactly in the same way as we did in our former version of the code. This results in the new composition $Y_{k}^{n+1}$ and electron fraction $Y_{\mathrm{e}}^{n+1}$.

We now transform the energy back to

$\varepsilon^{n+1}=e_{\mathrm{EoS}}^{n+1}+e_{\mathrm{kin}}^{n+1}-\sum_{k=1}^{n_{Y}} Y_{k}^{n+1} \cdot \frac{m_{k}}{m_{\mathrm{by}}} \cdot c^{2}-Y_{\mathrm{e}}^{n+1} \cdot \frac{m_{\mathrm{e}}}{m_{\mathrm{by}}} \cdot c^{2}-e_{0}$,

where we use $e_{\mathrm{rel}, 0}^{n+1}=e_{\mathrm{rel}, 0}^{*}$. By this simple treatment, we have implicitly taken into account the nuclear source term $Q_{\mathrm{nuc}}$ since

$\varepsilon^{n+1}-\varepsilon^{*}=-\sum_{k=1}^{n_{Y}}\left(Y_{k}^{n+1}-Y_{k}^{*}\right) \cdot \frac{m_{k}}{m_{\mathrm{by}}} \cdot c^{2}-\left(Y_{\mathrm{e}}^{n+1}-Y_{\mathrm{e}}^{*}\right) \cdot \frac{m_{e}}{m_{\mathrm{by}}} \cdot c^{2}=\frac{1}{\rho} Q_{\mathrm{nuc}} \Delta t^{n}$, 
where $\Delta t^{n}$ is the size of the time step $n$.

In other words, because we take into account effects which transform mass into internal energy and vice versa, i.e. composition changes due to nuclear transmutation and transformation of electrons to massless neutrinos, at a stage where the energy is defined including the rest masses of the particles which change abundance, Eq. (C.2), this energy is not affected by the transmutations and does not change. The conversion of energy from $e_{\text {int }}$ to $e_{\mathrm{rm}}$ or vice versa is not visible in their sum, $e_{\mathrm{rel}}=e_{\mathrm{int}}+e_{\mathrm{rm}}$. Although in NSE it is actually not necessary to advect the nuclear composition because it is unambiguously given for local thermodynamical conditions $\left(\rho, T, Y_{\mathrm{e}}\right)$ by the EoS, we follow the evolution equations (Eq. (C.6)) for a set of discrete nuclei which reproduce the baryon number and charge number of the representative heavy nucleus present at NSE in the employed high-density EoS (see Appendix B.1 in Rampp \& Janka 2002, which scheme was here extended by adding one more representative nucleus), in order to be able to subtract the rest-mass contribution to $e_{\text {rel, }, 0}$ (cf. Eq. (C.8)).

This procedure features several advantages: first, we do not need to calculate any nuclear source terms. This includes the NSE regime, where the nuclear composition and temperature are well defined functions of $\rho, Y_{\mathrm{e}}$, and $e_{\mathrm{rel}, 0}$ so that an iteration between $T, e_{\mathrm{int}}$, and the composition becomes unnecessary. Also in a regime where nuclear burning applies, the conservation of $e_{\mathrm{rel}, 0}$ ensures strict energy conservation in accordance with the philosophy of PROMETHEUS. Second, problems which may arise from the fact that the nucleon masses are not equal to their masses in vacuum at high densities because of nucleon-nucleon interactions are avoided by our implementation. For the energy transformations, Eqs. (C.8), (C.9), we can safely use the vacuum rest masses, because we need only ensure that $e_{\text {rel, }, 0}$ is correct. Its correctness is necessary to derive correct temperatures, composition, and the relativistic gravitational potential (via the gravitational mass, which includes terms depending on $e_{\mathrm{rel}}=e_{\mathrm{int}}+e_{\mathrm{rm}}$ in general relativity). At the high densities in the PNS, however, the use of $\varepsilon$ (Eq. (C.1)) without detailed knowledge of the effective nucleon masses once more leads to the problem that $\Gamma_{e}=\frac{p}{\left(\varepsilon-e_{\text {kin }}\right) \rho}+1$ may adopt values which are not perfectly consistent with the EoS index which relates gas pressure and internal energy density. However, this discrepancy now occurs at conditions where $\Gamma_{e} \gg 4 / 3$ and the relative error is much smaller than at low densities. Our test runs with the procedure described here confirm that the hydrodynamics results do not suffer from spurious oscillations anywhere in the SN core. 